\title{
Oxidation of Dipropyl Thiosulfinate Initiated by Cl Radicals in the Gas Phase: Implications for Atmospheric Chemistry
}

\author{
Parandaman Arathala and Rabi A. Musah* \\ University at Albany-State University of New York, Department of Chemistry, 1400 Washington Avenue, Albany, \\ NY 12222, USA \\ *Address correspondence to: rmusah@albany.edu
}

This section contains: Tables S1-S9 present the T1 diagnostic values, calculated total electronic energies including zero-point energy corrections, imaginary frequencies of various TSs at the M06-2X level, optimized geometries of all the stationary points, their vibrational frequencies and rotational constants, bimolecular rate coefficients in the absence and presence of tunneling contributions for each reaction path, and the optimized geometries of pre-reactive and post-reactive complexes associated with the DPTS $+{ }^{\circ} \mathrm{Cl}$ reaction. 
Table S1: T1 diagnostic values for all the stationary points involved in the DPTS $+{ }^{\circ} \mathrm{Cl}$ reaction system calculated at the $\operatorname{CCSD}(T) / c c-p V T Z$ level.

\begin{tabular}{|c|c|c|c|}
\hline Stationary point & T1 value & Stationary point & T1 value \\
\hline DPTS & 0.0142 & DPTS••Cl (PC6) & 0.0165 \\
\hline$\cdot \mathrm{Cl}$ & 0.0065 & DPTS ••Cl (TS5b) & 0.0150 \\
\hline $\mathrm{DPTS} \bullet \bullet \mathrm{Cl}(\mathrm{RC} 1)$ & 0.0159 & DPTS ••Cl (PC7) & 0.0165 \\
\hline DPTS ••Cl (TS1a) & 0.0186 & P5 & 0.0156 \\
\hline DPTS••Cl (PC1) & 0.0153 & DPTS ••Cl (TS6a) & 0.0150 \\
\hline $\mathrm{P} 1$ & 0.0168 & $\mathrm{DPTS} \bullet \mathrm{Cl}(\mathrm{PC} 8)$ & 0.0145 \\
\hline $\mathrm{HCl}$ & 0.0057 & DPTS $\bullet C l(R C 5)$ & 0.0145 \\
\hline $\mathrm{DPTS} \bullet \mathrm{Cl}(\mathrm{RC} 2)$ & 0.0171 & DPTS••Cl (PC9) & 0.0143 \\
\hline DPTS $\bullet C l(T S 1 b)$ & 0.0170 & P6 & 0.0149 \\
\hline DPTS••Cl (PC2) & 0.0162 & DPTS $\bullet C l(T S 6 b)$ & 0.0148 \\
\hline $\mathrm{DPTS} \bullet \bullet \mathrm{Cl}(\mathrm{RC} 3)$ & 0.0180 & $\mathrm{DPTS} \bullet \mathrm{Cl}(\mathrm{TS} 14)$ & 0.0149 \\
\hline DPTS ••Cl (TS2a) & 0.0151 & $\mathrm{DPTS} \bullet \mathrm{Cl}(\mathrm{PC} 10)$ & 0.0143 \\
\hline DPTS••Cl (PC3) & 0.0148 & DPTS • Cl (TS7a) & 0.0020 \\
\hline $\mathrm{P} 2$ & 0.0161 & $\mathrm{DPTS} \bullet \mathrm{Cl}(\mathrm{PC} 11)$ & 0.0143 \\
\hline DPTS $\bullet C l(T S 2 b)$ & 0.0153 & $\mathrm{CH}_{3} \mathrm{CH}_{2} \mathrm{CH}_{2} \mathrm{SCl}(\mathrm{P} 7 \mathrm{~b})$ & 0.0097 \\
\hline $\mathrm{DPTS} \bullet \mathrm{Cl}(\mathrm{RC} 4)$ & 0.0140 & $\mathrm{CH}_{3} \mathrm{CH}_{2} \mathrm{CH}_{2} \mathrm{~S} .(=\mathrm{O})(\mathrm{P} 7 \mathrm{a})$ & 0.0190 \\
\hline DPTS $\bullet C l(T S 3 a)$ & 0.0149 & DPTS••Cl (TS7b) & 0.0228 \\
\hline DPTS••Cl (PC4) & 0.0142 & $\mathrm{DPTS} \bullet \mathrm{Cl}(\mathrm{PC} 12)$ & 0.0139 \\
\hline $\mathrm{P} 3$ & 0.0148 & DPTS $\bullet C l(T S 8)$ & 0.0163 \\
\hline DPTS ••Cl (TS3b) & 0.0150 & $\mathrm{DPTS} \bullet \mathrm{Cl}(\mathrm{PC} 13)$ & 0.0139 \\
\hline DPTS $\bullet C l(T S 3 c)$ & 0.0149 & $\mathrm{CH}_{3} \mathrm{CH}_{2} \mathrm{CH}_{2} \mathrm{~S}(\mathrm{P} 8 \mathrm{~b})$ & 0.0106 \\
\hline DPTS ••Cl (TS4a) & 0.0165 & $\mathrm{CH}_{3} \mathrm{CH}_{2} \mathrm{CH}_{2} \mathrm{~S}(\mathrm{O}) \mathrm{Cl}(\mathrm{P} 8 \mathrm{a})$ & 0.0153 \\
\hline DPTS $\bullet C l(P C 5)$ & 0.0143 & DPTS • Cl (TS9) & 0.0185 \\
\hline P4 & 0.0178 & $\mathrm{DPTS} \bullet \mathrm{Cl}(\mathrm{PC} 14)$ & 0.0155 \\
\hline DPTS $\bullet C l(T S 4 b)$ & 0.0175 & $\mathrm{CH}_{3} \mathrm{CH}_{2} \mathrm{CH}_{2} \mathrm{SSOCl}(\mathrm{P} 9 \mathrm{a})$ & 0.0169 \\
\hline DPTS ••Cl (TS5a) & 0.0150 & $\mathrm{CH}_{3} \mathrm{CH}_{2} \mathrm{C}^{\circ} \mathrm{H}_{2}(\mathrm{P} 9 \mathrm{~b})$ & 0.0099 \\
\hline
\end{tabular}

Table S2: Calculated total electronic energies for reactants, pre-reactive complexes (RCs), transition states (TS), product complexes (PCs), and products at the M06-2X, and CCSD(T) levels. Zero-point energy (ZPE) corrections are given at the M06-2X level.

\begin{tabular}{|c|c|c|c|}
\hline Species & M06-2X/aug-cc-pVTZ & ZPE (M06-2X) & CCSD(T)/cc-pVTZ \\
\hline DPTS & -1108.62783 & 0.196672 & -1107.207676 \\
\hline$\cdot C l$ & -460.141208 & & -459.6718083 \\
\hline DPTS $\bullet C l$ (RC1) & -1568.800136 & 0.197766 & -1566.902860 \\
\hline DPTS $\bullet C l$ (TS1a) & -1568.768489 & 0.189319 & -1566.873467 \\
\hline
\end{tabular}




\begin{tabular}{|c|c|c|c|}
\hline $\mathrm{DPTS} \bullet \bullet \mathrm{Cl}(\mathrm{PC} 1)$ & -1568.786357 & 0.191466 & -1566.892441 \\
\hline $\mathrm{CH}_{3} \mathrm{CH}_{2} \mathrm{C}^{\cdot} \mathrm{H}-\mathrm{S}(\mathrm{O})-\mathrm{S}-\mathrm{CH}_{2} \mathrm{CH}_{2} \mathrm{CH}_{3}(\mathrm{P} 1)$ & -1107.964583 & 0.182697 & -1106.542581 \\
\hline $\mathrm{HCl}$ & -460.807507 & 0.006803 & -460.3372135 \\
\hline $\mathrm{DPTS} \bullet \cdot \mathrm{Cl}(\mathrm{RC} 2)$ & -1568.798598 & 0.197804 & -1566.901338 \\
\hline $\mathrm{DPTS} \cdot \bullet \mathrm{Cl}(\mathrm{TS} 1 \mathrm{~b})$ & -1568.764249 & 0.188849 & -1566.870500 \\
\hline $\mathrm{DPTS} \bullet \cdot \mathrm{Cl}(\mathrm{PC} 2)$ & -1568.779395 & 0.191425 & -1566.885827 \\
\hline DPTS $\bullet C l(\mathrm{RC} 3)$ & -1568.786801 & 0.197660 & -1566.891071 \\
\hline $\mathrm{DPTS} \bullet \cdot \mathrm{Cl}(\mathrm{TS} 2 \mathrm{a})$ & -1568.76547 & 0.188760 & -1566.870563 \\
\hline DPTS••Cl (PC3) & -1568.789313 & 0.190432 & -1566.894443 \\
\hline $\mathrm{CH}_{3} \mathrm{C}^{\cdot} \mathrm{HCH}_{2}-\mathrm{S}(\mathrm{O})-\mathrm{S}-\mathrm{CH}_{2} \mathrm{CH}_{2} \mathrm{CH}_{3}(\mathrm{P} 2)$ & -1107.966304 & 0.181733 & -1106.542946 \\
\hline DPTS $\bullet C l(T S 2 b)$ & -1568.770745 & 0.188755 & -1566.875881 \\
\hline $\mathrm{DPTS} \bullet \mathrm{Cl}(\mathrm{RC} 4)$ & -1568.772777 & 0.196829 & -1566.880602 \\
\hline DPTS $\bullet \cdot C l(T S 3 a)$ & -1568.766736 & 0.188923 & -1566.872182 \\
\hline DPTS ••Cl (PC4) & -1568.771031 & 0.190800 & -1566.877358 \\
\hline $\mathrm{C}^{\circ} \mathrm{H}_{2} \mathrm{CH}_{2} \mathrm{CH}_{2}-\mathrm{S}(\mathrm{O})-\mathrm{S}-\mathrm{CH}_{2} \mathrm{CH}_{2} \mathrm{CH}_{3}(\mathrm{P} 3)$ & -1107.956892 & 0.181374 & -1106.534677 \\
\hline DPTS $\bullet C l(T S 3 b)$ & -1568.764443 & 0.188992 & -1568.764443 \\
\hline DPTS $\bullet C l(T S 3 c)$ & -1568.764982 & 0.188782 & -1568.764982 \\
\hline DPTS $\bullet C l(T S 4 a)$ & -1568.771355 & 0.194587 & -1566.878382 \\
\hline DPTS••Cl (PC5) & -1568.792758 & 0.191442 & -1566.898640 \\
\hline $\mathrm{CH}_{3} \mathrm{CH}_{2} \mathrm{CH}_{2} \mathrm{~S}(\mathrm{O}) \mathrm{SC}^{\cdot} \mathrm{HCH}_{2} \mathrm{CH}_{3}(\mathrm{P} 4)$ & -1107.967813 & 0.182363 & -1106.545184 \\
\hline DPTS $\bullet \bullet C l(T S 4 b)$ & -1568.773007 & 0.190084 & -1566.877394 \\
\hline DPTS $\bullet C l(T S 5 a)$ & -1568.768540 & 0.192124 & -1566.877067 \\
\hline DPTS••Cl (PC6) & -1568.777000 & 0.190348 & -1566.881976 \\
\hline $\mathrm{CH}_{3} \mathrm{CH}_{2} \mathrm{CH}_{2} \mathrm{~S}(\mathrm{O}) \mathrm{SCH}_{2} \mathrm{C}^{\cdot} \mathrm{HCH}_{3}(\mathrm{P} 5)$ & -1107.965696 & 0.182294 & -1106.542048 \\
\hline DPTS $\bullet \bullet C l(T S 5 b)$ & -1568.771482 & 0.188985 & -1568.771482 \\
\hline $\mathrm{DPTS} \bullet \mathrm{Cl}(\mathrm{PC} 7)$ & -1568.776461 & 0.190644 & -1566.881346 \\
\hline $\mathrm{DPTS} \bullet \cdot \mathrm{Cl}(\mathrm{RC} 5)$ & -1568.801181 & 0.197836 & -1568.801181 \\
\hline DPTS $\bullet C l(T S 6 a)$ & -1568.765548 & 0.189106 & -1566.871325 \\
\hline DPTS••Cl (PC8) & -1568.770369 & 0.190602 & -1566.87714 \\
\hline $\mathrm{CH}_{3} \mathrm{CH}_{2} \mathrm{CH}_{2} \mathrm{~S}(\mathrm{O}) \mathrm{SCH}_{2} \mathrm{CH}_{2} \mathrm{C}^{\circ} \mathrm{H}_{2}(\mathrm{P} 6)$ & -1107.957314 & 0.181576 & -1106.535126 \\
\hline DPTS $\bullet \bullet C l(T S 6 b)$ & -1568.767137 & 0.189123 & -1566.872426 \\
\hline $\mathrm{DPTS} \bullet \mathrm{Cl}(\mathrm{PC} 9)$ & -1568.771389 & 0.190694 & -1566.877542 \\
\hline DPTS $\bullet C l(T S 6 c)$ & -1568.765242 & 0.189151 & -1566.870746 \\
\hline DPTS $\bullet C l(T S 7 a)$ & -1568.774821 & 0.196431 & -1566.876240 \\
\hline DPTS $\bullet C l(P C 11)$ & -1568.818074 & 0.196701 & -1566.921000 \\
\hline $\mathrm{CH}_{3} \mathrm{CH}_{2} \mathrm{CH}_{2} \mathrm{SCl}(\mathrm{P} 7 \mathrm{~b})$ & -976.914145 & 0.097068 & -975.7783153 \\
\hline $\mathrm{CH}_{3} \mathrm{CH}_{2} \mathrm{CH}_{2} \mathrm{~S}^{\circ}(=\mathrm{O})(\mathrm{P} 7 \mathrm{a})$ & -591.894681 & 0.098345 & -591.1342209 \\
\hline DPTS $\bullet \bullet C l(T S 7 b)$ & -1568.780579 & 0.196669 & -1566.881731 \\
\hline DPTS $\bullet \cdot C l(P C 12)$ & -1568.81673 & 0.196256 & -1566.919188 \\
\hline $\mathrm{DPTS} \bullet \mathrm{Cl}(\mathrm{TS} 8)$ & -1568.793519 & 0.196770 & -1566.899056 \\
\hline DPTS••Cl (PC13) & -1568.796315 & 0.196449 & -1566.902844 \\
\hline $\mathrm{CH}_{3} \mathrm{CH}_{2} \mathrm{CH}_{2} \mathrm{~S}^{\bullet}(\mathrm{P} 8 \mathrm{~b})$ & -516.664729 & 0.093531 & -516.0067573 \\
\hline $\mathrm{CH}_{3} \mathrm{CH}_{2} \mathrm{CH}_{2} \mathrm{~S}(\mathrm{O}) \mathrm{Cl}(\mathrm{P} 8 \mathrm{a})$ & -1052.122143 & 0.101134 & -1050.887383 \\
\hline DPTS $\bullet C l(T S 9)$ & -1568.767992 & 0.194536 & -1566.872269 \\
\hline DPTS $\bullet \cdot C l(\mathrm{PC} 14)$ & -1568.772054 & 0.193018 & -1566.875765 \\
\hline
\end{tabular}




\begin{tabular}{|c|c|c|c|}
\hline $\mathrm{CH}_{3} \mathrm{CH}_{2} \mathrm{CH}_{2} \mathrm{SSOCl}(\mathrm{P9a})$ & -1450.321304 & 0.102853 & -1448.628879 \\
\hline $\mathrm{CH}_{3} \mathrm{CH}_{2} \mathrm{CH}_{2}(\mathrm{P} 9 \mathrm{~b})$ & -118.443413 & 0.088358 & -118.2416773 \\
\hline
\end{tabular}

Table S3: Imaginary frequencies of all transition states at the M06-2X/6-311++G(3df,3pd) level of theory.

\begin{tabular}{|c|c|}
\hline Transition State & M06-2X/aug-cc-pVTZ \\
\hline DPTS $\bullet C l(T S 1 a)$ & 782 \\
\hline DPTS••Cl (TS1b) & 636 \\
\hline DPTS $\bullet C l(T S 2 a)$ & 974 \\
\hline DPTS••Cl (TS2b) & 706 \\
\hline DPTS••Cl (TS3a) & 637 \\
\hline DPTS••Cl (TS3b) & 698 \\
\hline DPTS $\bullet \mathrm{Cl}(\mathrm{TS} 3 \mathrm{c})$ & 679 \\
\hline DPTS••Cl (TS4a) & 182 \\
\hline DPTS $\bullet C l(T S 4 b)$ & 336 \\
\hline DPTS $\bullet C l(T S 5 a)$ & 204 \\
\hline DPTS $\bullet \cdot C l(T S 5 b)$ & 729 \\
\hline DPTS••Cl (TS6a) & 663 \\
\hline DPTS••Cl (TS6b) & 621 \\
\hline DPTS $\bullet \cdot \mathrm{Cl}(\mathrm{TS} 6 \mathrm{c})$ & 698 \\
\hline DPTS $\bullet \cdot C l(T S 7 a)$ & 1242 \\
\hline DPTS••Cl (TS7b) & 713 \\
\hline DPTS $\bullet C l(T S 8)$ & 95 \\
\hline DPTS $\bullet C l(T S 9)$ & 232 \\
\hline
\end{tabular}

Table S4: Calculated rotational constants $(\mathrm{GHz})$ for reactants, products, complexes, and transition states (TS) at the M06-2X level of theory.

\begin{tabular}{|c|c|c|c|}
\hline Species & $\mathbf{A}$ & B & C \\
\hline DPTS & 3.00 & 0.47 & 0.45 \\
\hline $\mathrm{DPTS} \bullet \cdot \mathrm{Cl}(\mathrm{RC} 1)$ & 1.12 & 0.47 & 0.37 \\
\hline DPTS ••Cl (TS1a) & 1.32 & 0.39 & 0.34 \\
\hline $\mathrm{DPTS} \bullet \mathrm{Cl}(\mathrm{PC} 1)$ & 1.01 & 0.50 & 0.41 \\
\hline $\mathrm{CH}_{3} \mathrm{CH}_{2} \mathrm{C}^{*} \mathrm{H}-\mathrm{S}(\mathrm{O})-\mathrm{S}_{-} \mathrm{CH}_{2} \mathrm{CH}_{2} \mathrm{CH}_{3}(\mathrm{P} 1)$ & 2.71 & 0.56 & 0.52 \\
\hline $\mathrm{HCl}$ & 315.26 & & \\
\hline $\mathrm{DPTS} \bullet \bullet \mathrm{Cl}(\mathrm{RC} 2)$ & 1.30 & 0.45 & 0.38 \\
\hline DPTS $\bullet$ Cl (TS1b) & 1.21 & 0.41 & 0.34 \\
\hline $\mathrm{DPTS} \bullet \mathrm{Cl}(\mathrm{PC} 2)$ & 0.76 & 0.60 & 0.38 \\
\hline $\mathrm{DPTS} \bullet \bullet \mathrm{Cl}(\mathrm{RC} 3)$ & 1.27 & 0.44 & 0.39 \\
\hline
\end{tabular}




\begin{tabular}{|c|c|c|c|}
\hline DPTS••Cl (TS2a) & 1.65 & 0.31 & 0.29 \\
\hline DPTS $\bullet C l(P C 3)$ & 1.16 & 0.42 & 0.36 \\
\hline $\mathrm{CH}_{3} \mathrm{C}^{\cdot} \mathrm{HCH}_{2}-\mathrm{S}(\mathrm{O})-\mathrm{S}_{-} \mathrm{CH}_{2} \mathrm{CH}_{2} \mathrm{CH}_{3}(\mathrm{P} 2)$ & 2.82 & 0.52 & 0.48 \\
\hline DPTS $\bullet C l(T S 2 b)$ & 1.22 & 0.37 & 0.32 \\
\hline DPTS••Cl (RC4) & 1.22 & 0.31 & 0.28 \\
\hline DPTS••Cl (TS3a) & 1.43 & 0.29 & 0.26 \\
\hline DPTS••Cl (PC4) & 1.20 & 0.31 & 0.27 \\
\hline $\mathrm{C}^{\circ} \mathrm{H}_{2} \mathrm{CH}_{2} \mathrm{CH}_{2}-\mathrm{S}(\mathrm{O})-\mathrm{S}-\mathrm{CH}_{2} \mathrm{CH}_{2} \mathrm{CH}_{3}(\mathrm{P} 3)$ & 3.01 & 0.48 & 0.46 \\
\hline DPTS $\bullet C l(T S 3 b)$ & 2.28 & 0.22 & 0.21 \\
\hline $\mathrm{DPTS} \bullet \bullet \mathrm{Cl}(\mathrm{TS} 3 \mathrm{c})$ & 1.82 & 0.28 & 0.26 \\
\hline DPTS••Cl (TS4a) & 1.29 & 0.36 & 0.32 \\
\hline DPTS••Cl (PC5) & 1.07 & 0.50 & 0.40 \\
\hline $\mathrm{CH}_{3} \mathrm{CH}_{2} \mathrm{CH}_{2} \mathrm{~S}(\mathrm{O}) \mathrm{SC}^{\cdot} \mathrm{HCH}_{2} \mathrm{CH}_{3}(\mathrm{P} 4)$ & 3.08 & 0.48 & 0.45 \\
\hline DPTS $\bullet \mathrm{Cl}(\mathrm{TS} 4 \mathrm{~b})$ & 1.54 & 0.33 & 0.29 \\
\hline DPTS••Cl (TS5a) & 1.09 & 0.46 & 0.37 \\
\hline DPTS••Cl (PC6) & 1.41 & 0.32 & 0.29 \\
\hline $\mathrm{CH}_{3} \mathrm{CH}_{2} \mathrm{CH}_{2} \mathrm{~S}(\mathrm{O}) \mathrm{SCH}_{2} \mathrm{C}^{\circ} \mathrm{HCH}_{3}(\mathrm{P} 5)$ & 2.45 & 0.58 & 0.52 \\
\hline DPTS•・Cl (RC5) & 1.25 & 0.45 & 0.38 \\
\hline DPTS $\bullet C l(T S 5 b)$ & 1.54 & 0.31 & 0.28 \\
\hline DPTS••Cl (PC7) & 1.40 & 0.31 & 0.27 \\
\hline DPTS••Cl (TS6a) & 1.51 & 0.23 & 0.23 \\
\hline DPTS••Cl (PC8) & 1.21 & 0.26 & 0.23 \\
\hline $\mathrm{CH}_{3} \mathrm{CH}_{2} \mathrm{CH}_{2} \mathrm{~S}(\mathrm{O}) \mathrm{SCH}_{2} \mathrm{CH}_{2} \mathrm{C}^{\circ} \mathrm{H}_{2}(\mathrm{P} 6)$ & 3.04 & 0.48 & 0.46 \\
\hline DPTS $\bullet$ Cl (TS6b) & 2.63 & 0.24 & 0.23 \\
\hline DPTS••Cl (PC9) & 2.17 & 0.25 & 0.23 \\
\hline DPTS ••Cl (TS6c) & 1.29 & 0.34 & 0.29 \\
\hline $\mathrm{DPTS} \bullet \bullet \mathrm{Cl}(\mathrm{PC} 10)$ & 1.14 & 0.41 & 0.33 \\
\hline $\mathrm{DPTS} \bullet \mathrm{Cl}(\mathrm{TS} 7 \mathrm{a})$ & 1.25 & 0.43 & 0.37 \\
\hline $\mathrm{DPTS} \bullet \bullet \mathrm{Cl}(\mathrm{PC} 11)$ & 1.25 & 0.45 & 0.39 \\
\hline $\mathrm{CH}_{3} \mathrm{CH}_{2} \mathrm{CH}_{2} \mathrm{SCl}(\mathrm{P} 7 \mathrm{~b})$ & 5.94 & 1.55 & 1.30 \\
\hline $\mathrm{CH}_{3} \mathrm{CH}_{2} \mathrm{CH}_{2} \mathrm{~S}^{*}(=\mathrm{O})(\mathrm{P} 7 \mathrm{a})$ & 11.14 & 1.95 & 1.78 \\
\hline DPTS $\bullet \bullet C l(T S 7 b)$ & 1.36 & 0.43 & 0.37 \\
\hline $\mathrm{DPTS} \bullet \cdot \mathrm{Cl}(\mathrm{PC} 12)$ & 1.20 & 0.38 & 0.33 \\
\hline DPTS $\bullet C l(T S 8)$ & 1.22 & 0.43 & 0.36 \\
\hline DPTS••Cl (PC13) & 1.10 & 0.49 & 0.39 \\
\hline $\mathrm{CH}_{3} \mathrm{CH}_{2} \mathrm{CH}_{2} \mathrm{~S}^{\bullet}(\mathrm{P} 8 \mathrm{~b})$ & 26.78 & 2.41 & 2.30 \\
\hline $\mathrm{CH}_{3} \mathrm{CH}_{2} \mathrm{CH}_{2} \mathrm{~S}(=\mathrm{O}) \mathrm{Cl}(\mathrm{P} 8 \mathrm{a})$ & 3.40 & 1.45 & 1.18 \\
\hline DPTS $\bullet \mathrm{Cl}(\mathrm{TS} 9)$ & 1.25 & 0.43 & 0.36 \\
\hline $\mathrm{DPTS} \bullet \bullet \mathrm{Cl}(\mathrm{PC} 14)$ & 0.88 & 0.51 & 0.38 \\
\hline $\mathrm{CH}_{3} \mathrm{CH}_{2} \mathrm{CH}_{2} \mathrm{SS}(\mathrm{O}) \mathrm{Cl}(\mathrm{P} 9 \mathrm{a})$ & 1.93 & 0.99 & 0.86 \\
\hline $\mathrm{CH}_{3} \mathrm{CH}_{2} \mathrm{C}^{\cdot} \mathrm{H}_{2}(\mathrm{P} 9 \mathrm{~b})$ & 32.85 & 9.10 & 7.87 \\
\hline
\end{tabular}

Table S5: Calculated positive frequencies $\left(\mathrm{cm}^{-1}\right)$ for reactants, pre-reactive complexes, transition states (TSs), post-reactive complexes, and products at the M06-2X level of theory. 


\begin{tabular}{|c|c|c|c|c|c|c|}
\hline \multirow{2}{*}{$\begin{array}{l}\text { Species } \\
\text { DPTS }\end{array}$} & \multicolumn{6}{|c|}{ Frequencies } \\
\hline & 18 & 52 & 75 & 86 & 94 & 117 \\
\hline & 189 & 240 & 248 & 251 & 258 & 297 \\
\hline & 341 & 378 & 414 & 503 & 723 & 734 \\
\hline & 747 & 749 & 856 & 865 & 918 & 923 \\
\hline & 1060 & 1060 & 1069 & 1082 & 1117 & 1122 \\
\hline & 1130 & 1239 & 1250 & 1252 & 1268 & 1329 \\
\hline & 1331 & 1372 & 1376 & 1414 & 1415 & 1450 \\
\hline & 1473 & 1495 & 1499 & 1503 & 1504 & 1511 \\
\hline & 1512 & 3061 & 3061 & 3064 & 3067 & 3073 \\
\hline & 3077 & 3100 & 3107 & 3123 & & 3134 \\
\hline & 3139 & 3141 & 3141 & & & \\
\hline \multirow[t]{11}{*}{$\mathrm{DPTS} \bullet \bullet \mathrm{Cl}(\mathrm{RC} 1)$} & 27 & 55 & 64 & 82 & 90 & 93 \\
\hline & 100 & 118 & 212 & 232 & 248 & 251 \\
\hline & 260 & 284 & 300 & 334 & 390 & 394 \\
\hline & 454 & 723 & 730 & 743 & 750 & 857 \\
\hline & 865 & 920 & 921 & 1058 & 1060 & 1078 \\
\hline & 1189 & 1114 & 1119 & 1145 & 1243 & 1247 \\
\hline & 1261 & 1271 & 1329 & 1330 & 1374 & 1374 \\
\hline & 1414 & 1416 & 1446 & 1453 & 1491 & 1499 \\
\hline & 1503 & 1504 & 1509 & 1512 & 3062 & 3064 \\
\hline & 3066 & 3068 & 3069 & 3086 & 3103 & 3107 \\
\hline & 3134 & 3135 & 3142 & 3143 & 3145 & 3145 \\
\hline \multirow[t]{11}{*}{$\mathrm{DPTS} \bullet \cdot C l(\mathrm{TS} 1 \mathrm{a})$} & -782 & 26 & 35 & 57 & 80 & 80 \\
\hline & 91 & 122 & 136 & 184 & 234 & 242 \\
\hline & 243 & 255 & 299 & 339 & 380 & 418 \\
\hline & 435 & 508 & 725 & 733 & 744 & 753 \\
\hline & 855 & 867 & 918 & 922 & 943 & 1026 \\
\hline & 1060 & 1066 & 1081 & 1120 & 1124 & 1131 \\
\hline & 1156 & 1228 & 1250 & 1268 & 1285 & 1327 \\
\hline & 1371 & 1372 & 1415 & 1417 & 1468 & 1471 \\
\hline & 1494 & 1502 & 1504 & 1509 & 1510 & 3026 \\
\hline & 3061 & 3066 & 3070 & 3077 & 3099 & 3099 \\
\hline & 3123 & 3134 & 3135 & 3140 & 3142 & 3154 \\
\hline \multirow[t]{7}{*}{$\mathrm{DPTS} \bullet \cdot \mathrm{Cl}(\mathrm{PC} 1)$} & 17 & 43 & 52 & 61 & 66 & 81 \\
\hline & 91 & 136 & 172 & 206 & 220 & 241 \\
\hline & 252 & 272 & 304 & 357 & 383 & 437 \\
\hline & 491 & 537 & 644 & 671 & 684 & 725 \\
\hline & 739 & 811 & 853 & 921 & 933 & 1023 \\
\hline & 1051 & 1059 & 1072 & 1082 & 1121 & 1141 \\
\hline & 1247 & 1248 & 1269 & 1300 & 1329 & 1357 \\
\hline
\end{tabular}




\begin{tabular}{|c|c|c|c|c|c|c|}
\hline & $\begin{array}{l}1373 \\
1496 \\
3064 \\
3124\end{array}$ & $\begin{array}{l}1405 \\
1504 \\
3065 \\
3135\end{array}$ & $\begin{array}{l}1414 \\
1504 \\
3068 \\
3142\end{array}$ & $\begin{array}{l}1463 \\
1509 \\
3077 \\
3144\end{array}$ & $\begin{array}{l}1484 \\
2242 \\
3099 \\
3157\end{array}$ & $\begin{array}{l}1492 \\
3062 \\
3120 \\
3205\end{array}$ \\
\hline $\begin{array}{l}\mathrm{CH}_{3} \mathrm{CH}_{2} \mathrm{C} \cdot \mathrm{H}-\mathrm{S}(\mathrm{O})-\mathrm{S}-\mathrm{CH}_{2} \mathrm{CH}_{2} \mathrm{CH}_{3} \\
(\mathrm{P} 1)\end{array}$ & $\begin{array}{l}23 \\
204 \\
331 \\
726 \\
1031 \\
1136 \\
1340 \\
1494 \\
3065 \\
3119\end{array}$ & $\begin{array}{l}49 \\
224 \\
378 \\
744 \\
1059 \\
1248 \\
1370 \\
1497 \\
3066 \\
3133\end{array}$ & $\begin{array}{c}70 \\
241 \\
421 \\
806 \\
1061 \\
1258 \\
1402 \\
1504 \\
3073 \\
3138\end{array}$ & $\begin{array}{c}77 \\
253 \\
475 \\
853 \\
1075 \\
1262 \\
1413 \\
1507 \\
3074 \\
3141\end{array}$ & $\begin{array}{l}83 \\
267 \\
541 \\
918 \\
1121 \\
1315 \\
1471 \\
1510 \\
3098 \\
3151\end{array}$ & $\begin{array}{c}158 \\
299 \\
670 \\
925 \\
1126 \\
1326 \\
1474 \\
3060 \\
3118 \\
3220\end{array}$ \\
\hline $\mathrm{HCl}$ & 2986 & & & & & \\
\hline DPTS••Cl (RC2) & $\begin{array}{l}20 \\
115 \\
252 \\
513 \\
865 \\
1083 \\
1251 \\
1415 \\
1504 \\
3069 \\
3124\end{array}$ & $\begin{array}{c}34 \\
138 \\
257 \\
723 \\
918 \\
1117 \\
1267 \\
1415 \\
1504 \\
3076 \\
3135\end{array}$ & $\begin{array}{c}62 \\
186 \\
299 \\
736 \\
922 \\
1122 \\
1333 \\
1442 \\
1511 \\
3079 \\
3138\end{array}$ & $\begin{array}{c}68 \\
196 \\
344 \\
740 \\
1058 \\
1180 \\
1334 \\
1470 \\
1513 \\
3092 \\
3144\end{array}$ & $\begin{array}{l}80 \\
241 \\
375 \\
747 \\
1059 \\
1241 \\
1376 \\
1495 \\
3061 \\
3102 \\
3146\end{array}$ & $\begin{array}{l}91 \\
245 \\
406 \\
857 \\
1076 \\
1247 \\
1379 \\
1500 \\
3063 \\
3121 \\
3153\end{array}$ \\
\hline DPTS••Cl (TS1b) & $\begin{array}{l}-636 \\
90 \\
252 \\
504 \\
827 \\
1060 \\
1137 \\
1373 \\
1494 \\
3062 \\
3124\end{array}$ & $\begin{array}{l}2 \\
112 \\
260 \\
520 \\
833 \\
1067 \\
1237 \\
1377 \\
1502 \\
3068 \\
3125\end{array}$ & $\begin{array}{l}32 \\
127 \\
267 \\
716 \\
856 \\
1082 \\
1250 \\
1414 \\
1503 \\
3070 \\
3136\end{array}$ & $\begin{array}{l}57 \\
189 \\
341 \\
729 \\
919 \\
1108 \\
1268 \\
1416 \\
1508 \\
3079 \\
3141\end{array}$ & $\begin{array}{l}65 \\
240 \\
373 \\
743 \\
938 \\
1121 \\
1278 \\
1461 \\
1511 \\
3081 \\
3142\end{array}$ & $\begin{array}{l}78 \\
242 \\
409 \\
775 \\
995 \\
1134 \\
1329 \\
1472 \\
3022 \\
3102 \\
3152\end{array}$ \\
\hline $\mathrm{DPTS} \bullet \mathrm{Cl}(\mathrm{PC} 2)$ & $\begin{array}{l}17 \\
103 \\
250 \\
427 \\
745\end{array}$ & $\begin{array}{c}38 \\
115 \\
272 \\
470 \\
822\end{array}$ & $\begin{array}{l}59 \\
161 \\
304 \\
513 \\
857\end{array}$ & $\begin{array}{l}64 \\
177 \\
328 \\
525 \\
919\end{array}$ & $\begin{array}{l}80 \\
216 \\
374 \\
709 \\
938\end{array}$ & $\begin{array}{l}85 \\
240 \\
404 \\
725 \\
1033\end{array}$ \\
\hline
\end{tabular}




\begin{tabular}{|c|c|c|c|c|c|c|}
\hline & $\begin{array}{l}1053 \\
1245 \\
1374 \\
1495 \\
3060 \\
3121\end{array}$ & $\begin{array}{l}1061 \\
1248 \\
1408 \\
1503 \\
3064 \\
3133\end{array}$ & $\begin{array}{l}1078 \\
1267 \\
1413 \\
1504 \\
3069 \\
3141\end{array}$ & $\begin{array}{l}1121 \\
1295 \\
1471 \\
1510 \\
3074 \\
3143\end{array}$ & $\begin{array}{l}1148 \\
1329 \\
1482 \\
2649 \\
3094 \\
3149\end{array}$ & $\begin{array}{l}1151 \\
1366 \\
1493 \\
3045 \\
3097 \\
3203\end{array}$ \\
\hline DPTS••Cl (RC3) & $\begin{array}{l}22 \\
121 \\
256 \\
512 \\
867 \\
1084 \\
1258 \\
1415 \\
1504 \\
3066 \\
3129\end{array}$ & $\begin{array}{l}35 \\
154 \\
262 \\
722 \\
921 \\
1087 \\
1272 \\
1415 \\
1505 \\
3067 \\
3136\end{array}$ & $\begin{array}{c}55 \\
198 \\
307 \\
731 \\
923 \\
1118 \\
1329 \\
1446 \\
1509 \\
3076 \\
3137\end{array}$ & $\begin{array}{l}71 \\
240 \\
351 \\
737 \\
1022 \\
1123 \\
1333 \\
1464 \\
1510 \\
3080 \\
3142\end{array}$ & $\begin{array}{l}80 \\
243 \\
397 \\
745 \\
1060 \\
1238 \\
1373 \\
1491 \\
3062 \\
3101 \\
3145\end{array}$ & $\begin{array}{l}85 \\
250 \\
418 \\
856 \\
1062 \\
1250 \\
1376 \\
1496 \\
3063 \\
3112 \\
3149\end{array}$ \\
\hline DPTS••Cl (TS2a) & $\begin{array}{l}-974 \\
107 \\
247 \\
478 \\
841 \\
1060 \\
1150 \\
1373 \\
1478 \\
3045 \\
3113\end{array}$ & $\begin{array}{l}9 \\
117 \\
253 \\
513 \\
857 \\
1083 \\
1221 \\
1373 \\
1489 \\
3061 \\
3124\end{array}$ & $\begin{array}{l}23 \\
150 \\
290 \\
721 \\
916 \\
1089 \\
1246 \\
1408 \\
1494 \\
3067 \\
3124\end{array}$ & $\begin{array}{l}41 \\
187 \\
335 \\
732 \\
919 \\
1109 \\
1251 \\
1414 \\
1503 \\
3077 \\
3134\end{array}$ & $\begin{array}{l}55 \\
214 \\
375 \\
743 \\
952 \\
1121 \\
1270 \\
1432 \\
1511 \\
3100 \\
3140\end{array}$ & $\begin{array}{l}78 \\
238 \\
410 \\
747 \\
1000 \\
1140 \\
1329 \\
1474 \\
3040 \\
3105 \\
3156\end{array}$ \\
\hline DPTS••Cl (PC3) & $\begin{array}{l}10 \\
105 \\
249 \\
489 \\
766 \\
1037 \\
1204 \\
1386 \\
1482 \\
3062 \\
3124\end{array}$ & $\begin{array}{c}36 \\
109 \\
264 \\
495 \\
856 \\
1060 \\
1232 \\
1414 \\
1494 \\
3068 \\
3135\end{array}$ & $\begin{array}{c}48 \\
150 \\
322 \\
604 \\
871 \\
1083 \\
1251 \\
1417 \\
1503 \\
3071 \\
3141\end{array}$ & $\begin{array}{l}62 \\
171 \\
342 \\
641 \\
915 \\
1086 \\
1269 \\
1458 \\
1510 \\
3078 \\
3141\end{array}$ & $\begin{array}{c}76 \\
189 \\
388 \\
730 \\
919 \\
1121 \\
1328 \\
1472 \\
2117 \\
3089 \\
3142\end{array}$ & $\begin{array}{c}84 \\
238 \\
414 \\
745 \\
992 \\
1166 \\
1373 \\
1475 \\
3014 \\
3101 \\
3204\end{array}$ \\
\hline $\begin{array}{l}\mathrm{CH}_{3} \mathrm{C}^{\cdot} \mathrm{HCH}_{2}-\mathrm{S}(\mathrm{O})-\mathrm{S}-\mathrm{CH}_{2} \mathrm{CH}_{2} \mathrm{CH}_{3} \\
(\mathrm{P} 2)\end{array}$ & $\begin{array}{l}19 \\
158 \\
340\end{array}$ & $\begin{array}{l}42 \\
193 \\
387\end{array}$ & $\begin{array}{c}54 \\
241 \\
411\end{array}$ & $\begin{array}{l}71 \\
252 \\
483\end{array}$ & $\begin{array}{l}80 \\
266 \\
489\end{array}$ & $\begin{array}{l}114 \\
320 \\
606\end{array}$ \\
\hline
\end{tabular}




\begin{tabular}{|c|c|c|c|c|c|c|}
\hline & $\begin{array}{l}730 \\
995 \\
1162 \\
1371 \\
1476 \\
3061 \\
3123\end{array}$ & $\begin{array}{l}746 \\
1060 \\
1191 \\
1384 \\
1484 \\
3066 \\
3133\end{array}$ & $\begin{array}{l}856 \\
1060 \\
1227 \\
1413 \\
1494 \\
3069 \\
3139\end{array}$ & $\begin{array}{c}865 \\
1083 \\
1251 \\
1417 \\
1504 \\
3077 \\
3139\end{array}$ & $\begin{array}{l}916 \\
1122 \\
1268 \\
1455 \\
1510 \\
3083 \\
3141\end{array}$ & $\begin{array}{c}919 \\
1130 \\
1328 \\
1472 \\
3020 \\
3099 \\
3206\end{array}$ \\
\hline $\mathrm{DPTS} \bullet \bullet \mathrm{Cl}(\mathrm{TS} 2 \mathrm{~b})$ & $\begin{array}{l}-706 \\
104 \\
251 \\
505 \\
837 \\
1060 \\
1158 \\
1371 \\
1477 \\
3036 \\
3123\end{array}$ & $\begin{array}{c}14 \\
120 \\
265 \\
535 \\
857 \\
1079 \\
1229 \\
1373 \\
1489 \\
3061 \\
3123\end{array}$ & $\begin{array}{c}28 \\
133 \\
302 \\
715 \\
917 \\
1084 \\
1235 \\
1408 \\
1494 \\
3068 \\
3135\end{array}$ & $\begin{array}{l}43 \\
180 \\
336 \\
727 \\
920 \\
1111 \\
1250 \\
1414 \\
1503 \\
3078 \\
3139\end{array}$ & $\begin{array}{c}68 \\
197 \\
376 \\
733 \\
939 \\
1122 \\
1268 \\
1426 \\
1510 \\
3101 \\
3141\end{array}$ & $\begin{array}{c}81 \\
241 \\
408 \\
748 \\
976 \\
1131 \\
1328 \\
1474 \\
3034 \\
3111 \\
3157\end{array}$ \\
\hline DPTS••Cl (RC4) & $\begin{array}{l}15 \\
105 \\
252 \\
504 \\
861 \\
1082 \\
1253 \\
1411 \\
1496 \\
3063 \\
3117\end{array}$ & $\begin{array}{l}23 \\
116 \\
260 \\
724 \\
918 \\
1114 \\
1268 \\
1414 \\
1503 \\
3067 \\
3122\end{array}$ & $\begin{array}{l}34 \\
140 \\
299 \\
734 \\
920 \\
1122 \\
1329 \\
1449 \\
1509 \\
3072 \\
3134\end{array}$ & $\begin{array}{c}55 \\
189 \\
341 \\
748 \\
1056 \\
1129 \\
1331 \\
1473 \\
1511 \\
3077 \\
3138\end{array}$ & $\begin{array}{l}80 \\
240 \\
379 \\
751 \\
1060 \\
1235 \\
1373 \\
1476 \\
2999 \\
3094 \\
3139\end{array}$ & $\begin{array}{l}90 \\
243 \\
415 \\
856 \\
1069 \\
1250 \\
1375 \\
1495 \\
3061 \\
3100 \\
3143\end{array}$ \\
\hline DPTS $\bullet C l(T S 3 a)$ & $\begin{array}{l}-637 \\
99 \\
260 \\
507 \\
816 \\
1060 \\
1152 \\
1328 \\
1468 \\
3061 \\
3123\end{array}$ & $\begin{array}{c}14 \\
117 \\
304 \\
610 \\
856 \\
1074 \\
1215 \\
1372 \\
1474 \\
3067 \\
3123\end{array}$ & $\begin{array}{l}24 \\
145 \\
340 \\
716 \\
857 \\
1082 \\
1250 \\
1379 \\
1494 \\
3077 \\
3135\end{array}$ & $\begin{array}{l}46 \\
188 \\
376 \\
729 \\
901 \\
1099 \\
1256 \\
1413 \\
1503 \\
3077 \\
3140\end{array}$ & $\begin{array}{l}55 \\
239 \\
380 \\
740 \\
918 \\
1121 \\
1268 \\
1452 \\
1510 \\
3086 \\
3147\end{array}$ & $\begin{array}{l}78 \\
251 \\
422 \\
747 \\
952 \\
1127 \\
1306 \\
1462 \\
3015 \\
3101 \\
3220\end{array}$ \\
\hline DPTS••Cl (PC4) & $\begin{array}{l}16 \\
110\end{array}$ & $\begin{array}{l}30 \\
125\end{array}$ & $\begin{array}{l}37 \\
133\end{array}$ & $\begin{array}{l}56 \\
189\end{array}$ & $\begin{array}{l}78 \\
238\end{array}$ & $\begin{array}{c}87 \\
240\end{array}$ \\
\hline
\end{tabular}




\begin{tabular}{|c|c|c|c|c|c|c|}
\hline & $\begin{array}{l}252 \\
424 \\
748 \\
1060 \\
1161 \\
1373 \\
1473 \\
3061 \\
3123\end{array}$ & $\begin{array}{c}260 \\
488 \\
769 \\
1079 \\
1249 \\
1376 \\
1495 \\
3065 \\
3134\end{array}$ & $\begin{array}{l}304 \\
506 \\
857 \\
1092 \\
1252 \\
1414 \\
1503 \\
3067 \\
3139\end{array}$ & $\begin{array}{c}342 \\
602 \\
888 \\
1100 \\
1268 \\
1452 \\
1511 \\
3077 \\
3144\end{array}$ & $\begin{array}{c}380 \\
717 \\
919 \\
0 \quad 1122 \\
1302 \\
1468 \\
2722 \\
3077 \\
3160\end{array}$ & $\begin{array}{c}420 \\
732 \\
984 \\
1126 \\
1329 \\
1473 \\
3009 \\
3100 \\
3263\end{array}$ \\
\hline $\begin{array}{l}\mathrm{C}^{\circ} \mathrm{H}_{2} \mathrm{CH}_{2} \mathrm{CH}_{2}-\mathrm{S}(\mathrm{O})-\mathrm{S}^{-} \mathrm{CH}_{2} \mathrm{CH}_{2} \mathrm{CH}_{3} \\
(\mathrm{P} 3)\end{array}$ & $\begin{array}{l}20 \\
120 \\
342 \\
734 \\
982 \\
1130 \\
1328 \\
1473 \\
3060 \\
3123\end{array}$ & $\begin{array}{c}53 \\
189 \\
379 \\
740 \\
1060 \\
1175 \\
1368 \\
1482 \\
3066 \\
3134\end{array}$ & $\begin{array}{c}72 \\
236 \\
414 \\
747 \\
1075 \\
1236 \\
1371 \\
1493 \\
3077 \\
3139\end{array}$ & $\begin{array}{c}85 \\
251 \\
475 \\
856 \\
1086 \\
6 \\
1251 \\
11412 \\
1502 \\
3077 \\
3145\end{array}$ & $\begin{array}{c}94 \\
258 \\
505 \\
892 \\
1099 \\
1268 \\
1449 \\
1510 \\
3100 \\
3171\end{array}$ & $\begin{array}{r}105 \\
300 \\
724 \\
918 \\
1120 \\
1303 \\
1471 \\
2983 \\
3104 \\
3277\end{array}$ \\
\hline DPTS••Cl (TS3b) & $\begin{array}{l}-698 \\
95 \\
257 \\
504 \\
799 \\
1060 \\
1163 \\
1329 \\
1473 \\
3067 \\
3123\end{array}$ & $\begin{array}{l}18 \\
101 \\
286 \\
577 \\
843 \\
1061 \\
1234 \\
1349 \\
1495 \\
3071 \\
3124\end{array}$ & $\begin{array}{l}26 \\
110 \\
336 \\
709 \\
856 \\
1072 \\
1247 \\
1372 \\
1503 \\
3078 \\
3135\end{array}$ & $\begin{array}{l}34 \\
186 \\
375 \\
732 \\
893 \\
1085 \\
1251 \\
1415 \\
1504 \\
3081 \\
3140\end{array}$ & $\begin{array}{c}65 \\
241 \\
390 \\
745 \\
919 \\
1122 \\
1268 \\
1447 \\
1511 \\
3100 \\
3149\end{array}$ & $\begin{array}{l}81 \\
250 \\
405 \\
750 \\
1020 \\
1135 \\
1324 \\
1469 \\
3061 \\
3121 \\
3217\end{array}$ \\
\hline $\mathrm{DPTS} \bullet \bullet \mathrm{Cl}(\mathrm{TS} 3 \mathrm{c})$ & $\begin{array}{l}-679 \\
96 \\
257 \\
507 \\
796 \\
1059 \\
1159 \\
1327 \\
1470 \\
3061 \\
3122\end{array}$ & $\begin{array}{l}11 \\
119 \\
299 \\
588 \\
854 \\
1066 \\
1221 \\
1371 \\
1473 \\
3067 \\
3123\end{array}$ & $\begin{array}{l}26 \\
138 \\
339 \\
718 \\
867 \\
1081 \\
1243 \\
1376 \\
1494 \\
3077 \\
3134\end{array}$ & $\begin{array}{l}38 \\
189 \\
378 \\
726 \\
914 \\
1103 \\
1251 \\
1414 \\
1503 \\
3077 \\
3140\end{array}$ & $\begin{array}{l}58 \\
240 \\
386 \\
735 \\
918 \\
1121 \\
1268 \\
1453 \\
1511 \\
3099 \\
3145\end{array}$ & $\begin{array}{l}77 \\
250 \\
421 \\
748 \\
937 \\
1135 \\
1311 \\
1463 \\
2997 \\
3105 \\
3218\end{array}$ \\
\hline
\end{tabular}




\begin{tabular}{|c|c|c|c|c|c|c|}
\hline DPTS••Cl (TS4a) & $\begin{array}{l}-183 \\
84 \\
253 \\
495 \\
866 \\
1070 \\
1252 \\
1376 \\
1501 \\
3064 \\
3118\end{array}$ & $\begin{array}{c}18 \\
94 \\
254 \\
697 \\
921 \\
1109 \\
1255 \\
1416 \\
1504 \\
3065 \\
3131\end{array}$ & $\begin{array}{l}25 \\
133 \\
297 \\
727 \\
926 \\
1118 \\
1301 \\
1417 \\
1504 \\
3067 \\
3137\end{array}$ & $\begin{array}{l}56 \\
183 \\
336 \\
751 \\
1050 \\
1130 \\
1311 \\
1449 \\
1512 \\
3072 \\
3141\end{array}$ & $\begin{array}{l}67 \\
242 \\
378 \\
762 \\
1060 \\
1186 \\
1332 \\
1482 \\
2406 \\
3102 \\
3144 \\
3\end{array}$ & $\begin{array}{l}69 \\
249 \\
409 \\
850 \\
1061 \\
1240 \\
1372 \\
1498 \\
3062 \\
3106 \\
3145\end{array}$ \\
\hline DPTS••Cl (PC5) & $\begin{array}{l}19 \\
121 \\
257 \\
472 \\
793 \\
1059 \\
1239 \\
1376 \\
1498 \\
3062 \\
3134\end{array}$ & $\begin{array}{l}30 \\
138 \\
284 \\
507 \\
806 \\
1063 \\
1265 \\
1407 \\
1503 \\
3067 \\
3144\end{array}$ & $\begin{array}{l}51 \\
169 \\
300 \\
694 \\
871 \\
1084 \\
1269 \\
1414 \\
1504 \\
3070 \\
3146\end{array}$ & $\begin{array}{l}74 \\
194 \\
321 \\
709 \\
923 \\
1091 \\
1315 \\
1452 \\
1509 \\
3078 \\
3145\end{array}$ & 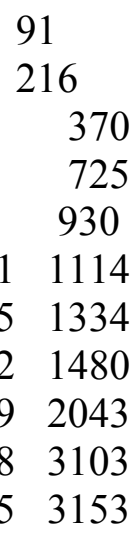 & $\begin{array}{rr}95 \\
245 \\
0 & 424 \\
5 & 744 \\
& 1034 \\
4 & 1118 \\
4 & 1336 \\
0 & 1493 \\
3 & 3025 \\
3 & 3114 \\
3 & 3211\end{array}$ \\
\hline $\mathrm{CH}_{3} \mathrm{CH}_{2} \mathrm{CH}_{2} \mathrm{~S}(\mathrm{O}) \mathrm{SC}^{\cdot} \mathrm{HCH}_{2} \mathrm{CH}_{3}(\mathrm{P} 4)$ & $\begin{array}{l}19 \\
155 \\
318 \\
750 \\
1060 \\
1142 \\
1375 \\
1498 \\
3062 \\
3131\end{array}$ & $\begin{array}{l}53 \\
232 \\
374 \\
759 \\
1066 \\
1239 \\
1378 \\
1502 \\
3063 \\
3135\end{array}$ & $\begin{array}{l}66 \\
239 \\
418 \\
809 \\
1071 \\
1254 \\
1412 \\
1504 \\
3065 \\
3141\end{array}$ & $\begin{array}{l}84 \\
250 \\
438 \\
865 \\
1074 \\
1261 \\
1415 \\
1506 \\
3066 \\
3141\end{array}$ & 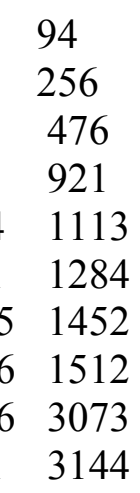 & $\begin{array}{cc} & 107 \\
& 290 \\
& 724 \\
& 959 \\
3 & 1137 \\
4 & 1331 \\
2 & 1472 \\
2 & 3000 \\
3 & 3107 \\
4 & 3197\end{array}$ \\
\hline DPTS ••Cl (TS4b) & $\begin{array}{l}-336 \\
93 \\
247 \\
449 \\
867 \\
1061 \\
1209 \\
1371 \\
1497 \\
3062 \\
3111\end{array}$ & $\begin{array}{l}9 \\
103 \\
252 \\
494 \\
879 \\
1071 \\
1238 \\
1376 \\
1501 \\
3065 \\
3132\end{array}$ & $\begin{array}{l}24 \\
145 \\
303 \\
726 \\
921 \\
1108 \\
1251 \\
1416 \\
1505 \\
3069 \\
3140\end{array}$ & $\begin{array}{l}57 \\
161 \\
329 \\
747 \\
931 \\
1117 \\
1255 \\
1417 \\
1510 \\
3074 \\
3143\end{array}$ & $\begin{array}{l}81 \\
232 \\
376 \\
752 \\
1015 \\
1140 \\
1299 \\
1449 \\
1511 \\
3093 \\
3144\end{array}$ & $\begin{array}{l}89 \\
244 \\
411 \\
828 \\
1060 \\
1162 \\
1332 \\
1476 \\
3050 \\
3107 \\
3155\end{array}$ \\
\hline
\end{tabular}




\begin{tabular}{|c|c|c|c|c|c|c|}
\hline DPTS••Cl (TS5a) & $\begin{array}{l}-204 \\
110 \\
256 \\
489 \\
869 \\
1072 \\
1219 \\
1375 \\
1496 \\
3061 \\
3121\end{array}$ & $\begin{array}{l}24 \\
116 \\
283 \\
691 \\
922 \\
1084 \\
1244 \\
1414 \\
1498 \\
3064 \\
3129\end{array}$ & $\begin{array}{l}34 \\
131 \\
307 \\
732 \\
926 \\
1115 \\
1251 \\
1416 \\
1503 \\
3066 \\
3133\end{array}$ & $\begin{array}{l}51 \\
211 \\
321 \\
752 \\
1038 \\
1122 \\
1254 \\
1441 \\
1512 \\
3076 \\
3141\end{array}$ & $\begin{array}{c}77 \\
223 \\
392 \\
760 \\
1062 \\
1143 \\
1331 \\
1449 \\
1581 \\
3107 \\
3148\end{array}$ & $\begin{array}{l}88 \\
245 \\
422 \\
866 \\
1065 \\
1154 \\
1361 \\
1480 \\
3054 \\
3109 \\
3148\end{array}$ \\
\hline $\mathrm{DPTS} \bullet \mathrm{Cl}(\mathrm{PC} 6)$ & $\begin{array}{l}20 \\
98 \\
247 \\
385 \\
748 \\
1060 \\
1183 \\
1389 \\
1487 \\
3016 \\
3109\end{array}$ & $\begin{array}{l}24 \\
112 \\
269 \\
414 \\
835 \\
1070 \\
1238 \\
1415 \\
1498 \\
3061 \\
3131\end{array}$ & $\begin{array}{c}42 \\
125 \\
285 \\
504 \\
866 \\
1099 \\
1253 \\
1419 \\
1504 \\
3065 \\
3139\end{array}$ & $\begin{array}{l}64 \\
138 \\
303 \\
513 \\
922 \\
1116 \\
1257 \\
1452 \\
1512 \\
3075 \\
3142\end{array}$ & $\begin{array}{c}78 \\
204 \\
335 \\
725 \\
927 \\
1129 \\
1331 \\
1453 \\
2823 \\
3077 \\
3143\end{array}$ & $\begin{array}{c}85 \\
236 \\
372 \\
730 \\
995 \\
1155 \\
1375 \\
1480 \\
3006 \\
3088 \\
3201\end{array}$ \\
\hline $\mathrm{CH}_{3} \mathrm{CH}_{2} \mathrm{CH}_{2} \mathrm{~S}(\mathrm{O}) \mathrm{SCH}_{2} \mathrm{C}^{\cdot} \mathrm{HCH}_{3}(\mathrm{P} 5)$ & $\begin{array}{l}37 \\
164 \\
341 \\
726 \\
992 \\
1168 \\
1375 \\
1483 \\
3062 \\
3128\end{array}$ & $\begin{array}{l}45 \\
180 \\
401 \\
750 \\
1060 \\
1220 \\
1392 \\
1498 \\
3065 \\
3131\end{array}$ & $\begin{array}{l}80 \\
249 \\
418 \\
853 \\
1070 \\
1239 \\
1415 \\
1500 \\
3068 \\
3141\end{array}$ & $\begin{array}{l}83 \\
256 \\
503 \\
866 \\
1082 \\
1241 \\
1417 \\
1504 \\
3073 \\
3142\end{array}$ & $\begin{array}{ll}97 \\
298 \\
532 \\
899 \\
1116 \\
1252 \\
7 & 1451 \\
4 & 1512 \\
3 & 3085 \\
2 & 3143\end{array}$ & $\begin{array}{c}127 \\
308 \\
604 \\
921 \\
1122 \\
1331 \\
1475 \\
3020 \\
3106 \\
3198\end{array}$ \\
\hline DPTS••Cl (RC5) & $\begin{array}{l}39 \\
113 \\
256 \\
479 \\
870 \\
1080 \\
1256 \\
1415 \\
1504 \\
3063 \\
3124\end{array}$ & $\begin{array}{c}49 \\
123 \\
280 \\
725 \\
919 \\
1113 \\
1262 \\
1415 \\
1504 \\
3067 \\
3135\end{array}$ & $\begin{array}{c}68 \\
184 \\
294 \\
732 \\
922 \\
1117 \\
1331 \\
1453 \\
1510 \\
3079 \\
3142\end{array}$ & $\begin{array}{c}86 \\
215 \\
331 \\
738 \\
1058 \\
1145 \\
1334 \\
1463 \\
1512 \\
3086 \\
3144\end{array}$ & $\begin{array}{c}93 \\
240 \\
377 \\
754 \\
1063 \\
1245 \\
1373 \\
1491 \\
3061 \\
3107 \\
3144\end{array}$ & $\begin{array}{c}108 \\
249 \\
409 \\
856 \\
1077 \\
1247 \\
1375 \\
1498 \\
3062 \\
3114 \\
3150\end{array}$ \\
\hline
\end{tabular}




\begin{tabular}{|c|c|c|c|c|c|c|}
\hline DPTS ••Cl (TS5b) & $\begin{array}{l}-729 \\
92 \\
253 \\
501 \\
837 \\
1061 \\
1157 \\
1372 \\
1478 \\
3038 \\
3111\end{array}$ & $\begin{array}{l}20 \\
113 \\
259 \\
518 \\
866 \\
1071 \\
1237 \\
1375 \\
1488 \\
3062 \\
3132\end{array}$ & $\begin{array}{l}35 \\
145 \\
297 \\
724 \\
919 \\
1095 \\
1241 \\
1406 \\
1498 \\
3066 \\
3133\end{array}$ & $\begin{array}{l}44 \\
181 \\
342 \\
738 \\
922 \\
1109 \\
1253 \\
1415 \\
1505 \\
3074 \\
3142\end{array}$ & $\begin{array}{l}73 \\
215 \\
381 \\
751 \\
947 \\
1117 \\
1260 \\
1443 \\
1512 \\
3105 \\
3143\end{array}$ & $\begin{array}{l}82 \\
250 \\
413 \\
753 \\
984 \\
1132 \\
1331 \\
1452 \\
3028 \\
3107 \\
3154\end{array}$ \\
\hline DPTS••Cl (PC7) & $\begin{array}{l}19 \\
94 \\
256 \\
413 \\
752 \\
1061 \\
1192 \\
1383 \\
1487 \\
3019 \\
3108\end{array}$ & $\begin{array}{c}31 \\
109 \\
266 \\
452 \\
838 \\
1072 \\
1241 \\
1415 \\
1498 \\
3063 \\
3132\end{array}$ & $\begin{array}{l}35 \\
116 \\
297 \\
491 \\
867 \\
1099 \\
1253 \\
1415 \\
1504 \\
3066 \\
3139\end{array}$ & $\begin{array}{l}57 \\
135 \\
339 \\
504 \\
921 \\
1117 \\
1257 \\
1452 \\
1512 \\
3074 \\
3142\end{array}$ & $\begin{array}{c}81 \\
195 \\
359 \\
725 \\
927 \\
1132 \\
1332 \\
1457 \\
2803 \\
3080 \\
3142\end{array}$ & $\begin{array}{c}86 \\
249 \\
384 \\
736 \\
997 \\
1153 \\
1376 \\
1480 \\
3010 \\
3095 \\
3191\end{array}$ \\
\hline DPTS••Cl (TS6a) & $\begin{array}{l}-663 \\
91 \\
257 \\
499 \\
812 \\
1060 \\
1166 \\
1332 \\
1474 \\
3065 \\
3121\end{array}$ & $\begin{array}{l}11 \\
100 \\
297 \\
579 \\
838 \\
1066 \\
1240 \\
1344 \\
1497 \\
3073 \\
3131\end{array}$ & $\begin{array}{l}22 \\
122 \\
338 \\
723 \\
865 \\
1069 \\
1250 \\
1376 \\
1498 \\
3076 \\
3141\end{array}$ & $\begin{array}{l}53 \\
199 \\
361 \\
735 \\
890 \\
1076 \\
1253 \\
1416 \\
1504 \\
3086 \\
3141\end{array}$ & $\begin{array}{l}65 \\
237 \\
391 \\
743 \\
922 \\
1117 \\
1266 \\
1449 \\
1512 \\
3106 \\
3143\end{array}$ & $\begin{array}{l}79 \\
249 \\
409 \\
749 \\
1011 \\
1131 \\
1323 \\
1464 \\
3061 \\
3121 \\
3215\end{array}$ \\
\hline DPTS••Cl (PC8) & $\begin{array}{l}9 \\
89 \\
256 \\
412 \\
748 \\
1061 \\
1239 \\
1334 \\
1493 \\
3064\end{array}$ & $\begin{array}{l}18 \\
97 \\
260 \\
472 \\
756 \\
1069 \\
1242 \\
1375 \\
1498 \\
3065\end{array}$ & $\begin{array}{c}36 \\
121 \\
297 \\
500 \\
839 \\
1071 \\
1251 \\
1414 \\
1504 \\
3073\end{array}$ & $\begin{array}{l}58 \\
174 \\
340 \\
625 \\
865 \\
1078 \\
1252 \\
1450 \\
1511 \\
3084\end{array}$ & $\begin{array}{l}74 \\
197 \\
365 \\
723 \\
922 \\
1116 \\
1327 \\
1463 \\
2752 \\
3107\end{array}$ & $\begin{array}{l}87 \\
245 \\
373 \\
732 \\
1005 \\
1131 \\
1331 \\
1475 \\
3062 \\
3109\end{array}$ \\
\hline
\end{tabular}




\begin{tabular}{|c|c|c|c|c|c|c|}
\hline & 3132 & 3139 & 3142 & 3142 & 3160 & 3261 \\
\hline \multirow{11}{*}{ DPTS••Cl (TS6b) } & -621 & 18 & 29 & 38 & 65 & 79 \\
\hline & 92 & 119 & 144 & 196 & 250 & 252 \\
\hline & 261 & 299 & 343 & 381 & 402 & 416 \\
\hline & 502 & 608 & 711 & 727 & 749 & 753 \\
\hline & 823 & 866 & 874 & 904 & 922 & 948 \\
\hline & 1061 & 1071 & 1078 & 1102 & 1117 & 1129 \\
\hline & 1156 & 1225 & 1241 & 1251 & 1275 & 1311 \\
\hline & 1332 & 1371 & 1376 & 1416 & 1449 & 1455 \\
\hline & 1465 & 1471 & 1498 & 1505 & 1511 & 3008 \\
\hline & 3062 & 3065 & 3073 & 3079 & 3097 & 3106 \\
\hline & 3123 & 3132 & 3135 & 3141 & 3142 & 3219 \\
\hline \multirow[t]{11}{*}{ DPTS••Cl (PC9) } & 16 & 24 & 36 & 56 & 76 & 90 \\
\hline & 102 & 120 & 130 & 194 & 242 & 248 \\
\hline & 254 & 263 & 297 & 342 & 385 & 406 \\
\hline & 417 & 484 & 502 & 601 & 717 & 729 \\
\hline & 748 & 766 & 865 & 886 & 921 & 981 \\
\hline & 1060 & 1071 & 1094 & 1099 & 1115 & 1130 \\
\hline & 1173 & 1239 & 1252 & 1272 & 1308 & 1330 \\
\hline & 1370 & 1375 & 1415 & 1449 & 1462 & 1468 \\
\hline & 1472 & 1497 & 1505 & 1511 & 2712 & 2997 \\
\hline & 3062 & 3065 & 3073 & 3075 & 3082 & 3106 \\
\hline & 3131 & 3131 & 3141 & 3142 & 3159 & 3260 \\
\hline \multirow[t]{10}{*}{$\mathrm{CH}_{3} \mathrm{CH}_{2} \mathrm{CH}_{2} \mathrm{~S}(\mathrm{O}) \mathrm{SCH}_{2} \mathrm{CH}_{2} \mathrm{C}^{\cdot} \mathrm{H}_{2}(\mathrm{P} 6)$} & 15 & 53 & 70 & 82 & 90 & 94 \\
\hline & 120 & 191 & 249 & 251 & 257 & 297 \\
\hline & 340 & 368 & 411 & 501 & 507 & 721 \\
\hline & 730 & 747 & 750 & 837 & 865 & 921 \\
\hline & 1004 & 1060 & 1068 & 1071 & 1085 & 1116 \\
\hline & 1128 & 1235 & 1240 & 1246 & 1253 & 1324 \\
\hline & 1331 & 1333 & 1375 & 1415 & 1451 & 1461 \\
\hline & 1473 & 1493 & 1498 & 1504 & 1512 & 3062 \\
\hline & 3062 & 3065 & 3074 & 3084 & 3107 & 3107 \\
\hline & 3131 & 3138 & 3142 & 3142 & 3174 & 3276 \\
\hline \multirow[t]{9}{*}{$\mathrm{DPTS} \bullet \mathrm{Cl}(\mathrm{TS} 6 \mathrm{c})$} & -698 & 18 & 29 & 45 & 65 & 89 \\
\hline & 99 & 120 & 155 & 194 & 250 & 251 \\
\hline & 258 & 296 & 336 & 376 & 399 & 421 \\
\hline & 499 & 587 & 715 & 729 & 750 & 754 \\
\hline & 806 & 866 & 884 & 914 & 923 & 943 \\
\hline & 1061 & 1070 & 1073 & 1106 & 1118 & 1142 \\
\hline & 1151 & 1223 & 1240 & 1253 & 1269 & 1312 \\
\hline & 1331 & 1375 & 1376 & 1416 & 1452 & 1458 \\
\hline & 1466 & 1479 & 1499 & 1505 & 1513 & 3019 \\
\hline
\end{tabular}




\begin{tabular}{|c|c|c|c|c|c|c|}
\hline & $\begin{array}{l}3062 \\
3120\end{array}$ & $\begin{array}{l}3065 \\
3128\end{array}$ & $\begin{array}{l}3075 \\
3131\end{array}$ & $\begin{array}{l}3076 \\
3142\end{array}$ & $\begin{array}{l}3085 \\
3142\end{array}$ & $\begin{array}{l}3109 \\
3216\end{array}$ \\
\hline \multirow[t]{11}{*}{$\mathrm{DPTS} \bullet \bullet \mathrm{Cl}(\mathrm{PC} 10)$} & 34 & 37 & 48 & 68 & 90 & 102 \\
\hline & 109 & 126 & 148 & 178 & 197 & 252 \\
\hline & 256 & 260 & 297 & 300 & 336 & 386 \\
\hline & 422 & 450 & 499 & 552 & 725 & 734 \\
\hline & 750 & 752 & 866 & 899 & 922 & 970 \\
\hline & 1060 & 1070 & 1092 & 1104 & 1117 & 1138 \\
\hline & 1161 & 1240 & 1253 & 1264 & 1303 & 1331 \\
\hline & 1374 & 1375 & 1416 & 1453 & 1465 & 1469 \\
\hline & 1474 & 1500 & 1505 & 1513 & 2841 & 3007 \\
\hline & 3061 & 3062 & 3065 & 3074 & 3079 & 3109 \\
\hline & 3128 & 3131 & 3141 & 3142 & 3159 & 3261 \\
\hline \multirow[t]{11}{*}{ DPTS••Cl (TS7a) } & -1242 & 23 & 37 & 65 & 80 & 91 \\
\hline & 101 & 106 & 145 & 180 & 208 & 242 \\
\hline & 249 & 250 & 298 & 317 & 335 & 362 \\
\hline & 413 & 724 & 740 & 744 & 761 & 862 \\
\hline & 865 & 917 & 917 & 1057 & 1058 & 1070 \\
\hline & 1080 & 1107 & 1110 & 1131 & 1241 & 1243 \\
\hline & 1246 & 1261 & 1325 & 1329 & 1371 & 1371 \\
\hline & 1414 & 1415 & 1447 & 1471 & 1495 & 1498 \\
\hline & 1504 & 1504 & 1510 & 1511 & 3061 & 3062 \\
\hline & 3064 & 3065 & 3070 & 3073 & 3099 & 3107 \\
\hline & 3130 & 3131 & 3138 & 3140 & 3141 & 3143 \\
\hline \multirow[t]{11}{*}{$\mathrm{DPTS} \bullet \mathrm{Cl}(\mathrm{PC} 11)$} & 21 & 36 & 46 & 56 & 74 & 90 \\
\hline & 98 & 108 & 122 & 144 & 172 & 227 \\
\hline & 244 & 258 & 289 & 311 & 345 & 414 \\
\hline & 545 & 722 & 740 & 754 & 781 & 852 \\
\hline & 884 & 914 & 919 & 1057 & 1060 & 1065 \\
\hline & 1078 & 1097 & 1106 & 1115 & 1239 & 1242 \\
\hline & 1253 & 1261 & 1324 & 1327 & 1371 & 1374 \\
\hline & 1411 & 1414 & 1443 & 1483 & 1496 & 1497 \\
\hline & 1502 & 1503 & 1510 & 1512 & 3061 & 3062 \\
\hline & 3064 & 3066 & 3072 & 3082 & 3092 & 3103 \\
\hline & 3124 & 3134 & 3135 & 3139 & 3141 & 3148 \\
\hline \multirow[t]{5}{*}{$\mathrm{CH}_{3} \mathrm{CH}_{2} \mathrm{CH}_{2} \mathrm{~S}^{-}(\mathrm{O})(\mathrm{P} 7 \mathrm{a})$} & 85 & 96 & 210 & 251 & 309 & 417 \\
\hline & 718 & 737 & 851 & 915 & 1055 & 1061 \\
\hline & 1100 & 1107 & 1242 & 1247 & 1327 & 1370 \\
\hline & 1415 & 1450 & 1497 & 1504 & 1511 & 3060 \\
\hline & 3061 & 3072 & 3105 & 3121 & 3135 & 3138 \\
\hline \multirow[t]{2}{*}{$\mathrm{CH}_{3} \mathrm{CH}_{2} \mathrm{CH}_{2} \mathrm{SCl}(\mathrm{P} 7 \mathrm{~b})$} & 83 & 105 & 214 & 242 & 265 & 386 \\
\hline & 532 & 722 & 745 & 854 & 920 & 1060 \\
\hline
\end{tabular}




\begin{tabular}{|c|c|c|c|c|c|c|}
\hline & $\begin{array}{l}1081 \\
1413 \\
3061\end{array}$ & $\begin{array}{l}1120 \\
1451 \\
3077\end{array}$ & $\begin{array}{l}1246 \\
1496 \\
3104\end{array}$ & $\begin{array}{c}1265 \\
1504 \\
3119\end{array}$ & $\begin{array}{c}1327 \\
1511 \\
3135\end{array}$ & $\begin{array}{c}1373 \\
3056 \\
3140\end{array}$ \\
\hline \multirow[t]{11}{*}{ DPTS••Cl (TS7b) } & -713 & 29 & 38 & 67 & 89 & 100 \\
\hline & 112 & 126 & 136 & 193 & 220 & 238 \\
\hline & 250 & 253 & 264 & 318 & 372 & 399 \\
\hline & 410 & 721 & 725 & 749 & 751 & 865 \\
\hline & 868 & 917 & 921 & 1059 & 1059 & 1073 \\
\hline & 1076 & 1108 & 1111 & 1132 & 1242 & 1244 \\
\hline & 1250 & 1261 & 1326 & 1330 & 1369 & 1371 \\
\hline & 1414 & 1415 & 1450 & 1465 & 1493 & 1497 \\
\hline & 1504 & 1504 & 1510 & 1511 & 3056 & 3062 \\
\hline & 3063 & 3065 & 3073 & 3081 & 3105 & 3105 \\
\hline & 3120 & 3132 & 3138 & 3141 & 3141 & 3143 \\
\hline \multirow{11}{*}{$\mathrm{DPTS} \cdot \bullet \mathrm{Cl}(\mathrm{PC} 12)$} & 18 & 40 & 41 & 48 & 67 & 80 \\
\hline & 87 & 100 & 103 & 115 & 217 & 227 \\
\hline & 240 & 244 & 267 & 311 & 386 & 419 \\
\hline & 529 & 714 & 722 & 739 & 743 & 852 \\
\hline & 854 & 915 & 920 & 1057 & 1060 & 1063 \\
\hline & 1081 & 1100 & 1108 & 1121 & 1242 & 1245 \\
\hline & 1250 & 1269 & 1326 & 1328 & 1371 & 1374 \\
\hline & 1413 & 1414 & 1442 & 1448 & 1495 & 1497 \\
\hline & 1503 & 1503 & 1510 & 1511 & 3061 & 3061 \\
\hline & 3064 & 3064 & 3072 & 3074 & 3105 & 3105 \\
\hline & 3124 & 3130 & 3136 & 3139 & 3139 & 3142 \\
\hline \multirow[t]{11}{*}{ DPTS••Cl (TS8) } & -95 & 16 & 35 & 51 & 72 & 77 \\
\hline & 92 & 106 & 180 & 193 & 243 & 244 \\
\hline & 247 & 252 & 282 & 322 & 373 & 401 \\
\hline & 493 & 706 & 715 & 741 & 743 & 841 \\
\hline & 862 & 917 & 925 & 1048 & 1058 & 1064 \\
\hline & 1073 & 1114 & 1117 & 1198 & 1231 & 1239 \\
\hline & 1257 & 1267 & 1325 & 1330 & 1366 & 1374 \\
\hline & 1412 & 1416 & 1447 & 1451 & 1490 & 1499 \\
\hline & 1503 & 1505 & 1508 & 1513 & 3047 & 3060 \\
\hline & 3064 & 3065 & 3079 & 3094 & 3096 & 3115 \\
\hline & 3121 & 3131 & 3137 & 3139 & 3143 & 3151 \\
\hline \multirow[t]{6}{*}{ DPTS••Cl (PC13) } & 9 & 16 & 36 & 53 & 67 & 77 \\
\hline & 105 & 126 & 153 & 179 & 223 & 244 \\
\hline & 260 & 262 & 296 & 324 & 346 & 442 \\
\hline & 491 & 651 & 699 & 741 & 761 & 796 \\
\hline & 859 & 902 & 924 & 1011 & 1054 & 1059 \\
\hline & 1070 & 1083 & 1117 & 1182 & 1236 & 1240 \\
\hline
\end{tabular}




\begin{tabular}{|c|c|c|c|c|c|c|}
\hline & $\begin{array}{l}1255 \\
1406 \\
1500 \\
3070 \\
3128\end{array}$ & $\begin{array}{l}1260 \\
1415 \\
1504 \\
3073 \\
3132\end{array}$ & $\begin{array}{l}1321 \\
1451 \\
1508 \\
3078 \\
3136\end{array}$ & $\begin{array}{c}1328 \\
1470 \\
1513 \\
3091 \\
3141\end{array}$ & $\begin{array}{l}1359 \\
1490 \\
3061 \\
3102 \\
3143\end{array}$ & $\begin{array}{c}1372 \\
1499 \\
3062 \\
3117 \\
3146\end{array}$ \\
\hline $\mathrm{CH}_{3} \mathrm{CH}_{2} \mathrm{CH}_{2} \mathrm{~S}(\mathrm{O}) \mathrm{Cl}(\mathrm{P} 8 \mathrm{a})$ & $\begin{array}{l}60 \\
324 \\
924 \\
1260 \\
1504 \\
3129\end{array}$ & $\begin{array}{l}99 \\
450 \\
1059 \\
1329 \\
1512 \\
3143\end{array}$ & $\begin{array}{l}178 \\
505 \\
1068 \\
1374 \\
3060 \\
3145\end{array}$ & $\begin{array}{c}242 \\
699 \\
1116 \\
1415 \\
3062\end{array}$ & $\begin{array}{l}253 \\
740 \\
1198 \\
1448 \\
3090\end{array}$ & $\begin{array}{c}291 \\
858 \\
1240 \\
1498 \\
3137\end{array}$ \\
\hline $\mathrm{CH}_{3} \mathrm{CH}_{2} \mathrm{CH}_{2} \mathrm{~S}^{\bullet}(\mathrm{P} 8 \mathrm{~b})$ & $\begin{array}{l}108 \\
768 \\
1252 \\
1503 \\
3106\end{array}$ & $\begin{array}{c}239 \\
918 \\
1317 \\
1509 \\
3129\end{array}$ & $\begin{array}{c}240 \\
967 \\
1365 \\
3039 \\
3138\end{array}$ & $\begin{array}{c}372 \\
1059 \\
1413 \\
3052\end{array}$ & $\begin{array}{c}401 \\
1120 \\
1433 \\
3058\end{array}$ & $\begin{array}{l}748 \\
1237 \\
1493 \\
3072\end{array}$ \\
\hline DPTS••Cl (TS9) & $\begin{array}{l}-232 \\
116 \\
249 \\
415 \\
851 \\
1058 \\
1249 \\
1400 \\
1500 \\
3070 \\
3134\end{array}$ & $\begin{array}{l}32 \\
130 \\
253 \\
472 \\
876 \\
1088 \\
1273 \\
1411 \\
1503 \\
3077 \\
3139\end{array}$ & $\begin{array}{c}43 \\
149 \\
264 \\
578 \\
907 \\
1116 \\
1318 \\
1468 \\
1509 \\
3079 \\
3140\end{array}$ & $\begin{array}{c}59 \\
170 \\
331 \\
721 \\
916 \\
1121 \\
1328 \\
1471 \\
1511 \\
3114 \\
3142\end{array}$ & $\begin{array}{c}61 \\
217 \\
357 \\
743 \\
919 \\
1194 \\
1333 \\
1492 \\
3060 \\
3118 \\
3143\end{array}$ & $\begin{array}{l}86 \\
237 \\
397 \\
755 \\
1038 \\
1206 \\
1373 \\
1495 \\
3063 \\
3125 \\
3230\end{array}$ \\
\hline DPTS••Cl (PC14) & $\begin{array}{l}33 \\
82 \\
247 \\
405 \\
757 \\
1059 \\
1250 \\
1399 \\
1499 \\
3060 \\
3132\end{array}$ & $\begin{array}{c}41 \\
90 \\
253 \\
438 \\
849 \\
1085 \\
1269 \\
1413 \\
1504 \\
3072 \\
3136\end{array}$ & $\begin{array}{l}52 \\
103 \\
261 \\
479 \\
896 \\
1107 \\
1309 \\
1451 \\
1509 \\
3081 \\
3141\end{array}$ & $\begin{array}{l}57 \\
175 \\
301 \\
598 \\
914 \\
1123 \\
1326 \\
1470 \\
1510 \\
3093 \\
3142\end{array}$ & $\begin{array}{l}69 \\
191 \\
344 \\
720 \\
921 \\
1195 \\
1333 \\
1492 \\
3053 \\
3113 \\
3160\end{array}$ & $\begin{array}{l}79 \\
230 \\
359 \\
734 \\
1043 \\
1203 \\
1376 \\
1493 \\
3059 \\
3126 \\
3260\end{array}$ \\
\hline $\mathrm{CH}_{3} \mathrm{CH}_{2} \mathrm{CH}_{2} \mathrm{SS}(\mathrm{O})-\mathrm{Cl}(\mathrm{P} 9 \mathrm{a})$ & $\begin{array}{l}58 \\
256 \\
721 \\
1123\end{array}$ & $\begin{array}{l}75 \\
299 \\
732 \\
1198\end{array}$ & $\begin{array}{l}89 \\
345 \\
850 \\
1251\end{array}$ & $\begin{array}{c}173 \\
408 \\
921 \\
1270\end{array}$ & $\begin{array}{c}225 \\
450 \\
1059 \\
1334\end{array}$ & $\begin{array}{c}248 \\
484 \\
1086 \\
1377\end{array}$ \\
\hline
\end{tabular}




\begin{tabular}{|l|llllll|}
\hline & 1414 & 1453 & 1492 & 1504 & 1509 & 3061 \\
& 3074 & 3082 & 3134 & 3129 & 3142 & 3143 \\
\hline propyl radical $\left(\mathrm{CH}_{3} \mathrm{CH}_{2} \mathrm{C}^{\circ} \mathrm{H}_{2},(\mathrm{P} 9 \mathrm{~b})\right)$ & 42 & 253 & 372 & 447 & 756 & 895 \\
& 928 & 1052 & 1101 & 1178 & 1271 & 1363 \\
& 1410 & 1471 & 1474 & 1502 & 1508 & 2985 \\
& 3058 & 3062 & 3129 & 3138 & 3165 & 3267 \\
\hline
\end{tabular}

Table S6: Z-matrices for the optimized structures of all species and complexes at the MO62X/aug-cc-pVTZ level of theory.

\section{DPTS}

$\begin{array}{lrrr}\mathrm{O} & 0.01640900 & 0.01031900 & -0.05795000 \\ \mathrm{~S} & -0.00769900 & 0.08723500 & 1.42856000 \\ \mathrm{C} & 1.72861200 & 0.04365800 & 1.97012600 \\ \mathrm{H} & 1.73580300 & 0.24301000 & 3.04272300 \\ \mathrm{H} & 2.23558000 & 0.85117600 & 1.44089700 \\ \mathrm{C} & 2.32552100 & -1.31341100 & 1.62991300 \\ \mathrm{H} & 2.20198200 & -1.49021100 & 0.56107400 \\ \mathrm{H} & 1.77131700 & -2.09658800 & 2.15138700 \\ \mathrm{C} & 3.80039300 & -1.37557100 & 2.00954100 \\ \mathrm{H} & 3.93811900 & -1.20994000 & 3.07852900 \\ \mathrm{H} & 4.22516000 & -2.34698000 & 1.76172500 \\ \mathrm{H} & 4.36943700 & -0.61358700 & 1.47651100 \\ \mathrm{C} & -1.95847900 & 2.27590100 & 1.32816300 \\ \mathrm{H} & -1.93131400 & 1.89183300 & 0.30714900 \\ \mathrm{H} & -2.12363300 & 3.35200200 & 1.26637000 \\ \mathrm{C} & -3.04284300 & 1.60378700 & 2.15494100 \\ \mathrm{H} & -2.82102900 & 0.53884200 & 2.24351200 \\ \mathrm{H} & -3.02662100 & 2.01302200 & 3.16592500 \\ \mathrm{C} & -4.41623600 & 1.79760000 & 1.52259300 \\ \mathrm{H} & -5.19275600 & 1.31571400 & 2.11472500 \\ \mathrm{H} & -4.66308800 & 2.85710500 & 1.44327700 \\ \mathrm{H} & -4.44541000 & 1.37209000 & 0.51912700 \\ \mathrm{~S} & -0.27787200 & 2.11207500 & 2.00752900\end{array}$

\section{$\underline{\text { DPTS } \bullet \text { Cl (RC1) }}$}

$\begin{array}{lrrr}\mathrm{O} & -0.68461700 & -0.71661200 & 0.91087800 \\ \mathrm{~S} & -0.21833500 & -0.50521100 & 2.30292700 \\ \mathrm{C} & 1.59244800 & -0.42781600 & 2.24524500 \\ \mathrm{H} & 1.91944400 & -0.06326700 & 3.22178400 \\ \mathrm{H} & 1.84134200 & 0.30990300 & 1.48224400 \\ \mathrm{C} & 2.13816100 & -1.81333300 & 1.92949100\end{array}$




$\begin{array}{lrrr}\mathrm{H} & 1.71373300 & -2.14996800 & 0.98324400 \\ \mathrm{H} & 1.82079700 & -2.52225000 & 2.69718800 \\ \mathrm{C} & 3.66006500 & -1.78345900 & 1.84203400 \\ \mathrm{H} & 4.10025400 & -1.46031900 & 2.78561600 \\ \mathrm{H} & 4.05295800 & -2.77099000 & 1.60643900 \\ \mathrm{H} & 3.98817500 & -1.09338500 & 1.06514700 \\ \mathrm{C} & -1.91353500 & 1.85640700 & 1.69830300 \\ \mathrm{H} & -1.63523300 & 1.43833800 & 0.73040300 \\ \mathrm{H} & -1.98157300 & 2.93937300 & 1.59683100 \\ \mathrm{C} & -3.19195000 & 1.23946800 & 2.24265600 \\ \mathrm{H} & -3.05147700 & 0.16339900 & 2.36054100 \\ \mathrm{H} & -3.40657500 & 1.64792000 & 3.23102300 \\ \mathrm{C} & -4.35407100 & 1.50329700 & 1.29154800 \\ \mathrm{H} & -5.27447900 & 1.06338000 & 1.67159600 \\ \mathrm{H} & -4.51831400 & 2.57366400 & 1.16428300 \\ \mathrm{H} & -4.15304300 & 1.07368100 & 0.31035000 \\ \mathrm{~S} & -0.48174700 & 1.61222100 & 2.77869300 \\ \mathrm{Cl} & 1.05208100 & 2.84383300 & 1.13686500\end{array}$

\section{$\underline{\text { DPTS } \bullet C I ~(T S 1 a) ~}$}

$\begin{array}{lrrr}\mathrm{O} & -0.56891700 & -0.01017000 & 0.88487100 \\ \mathrm{~S} & -0.73651200 & 0.31958100 & 2.31743000 \\ \mathrm{C} & 0.82259400 & 0.15063000 & 3.13662900 \\ \mathrm{H} & 0.71183200 & 0.30475500 & 4.20898400 \\ \mathrm{H} & 1.57828000 & 1.26072400 & 2.74842500 \\ \mathrm{C} & 1.64508800 & -1.01876200 & 2.67541900 \\ \mathrm{H} & 1.82457400 & -0.91243100 & 1.60440700 \\ \mathrm{H} & 1.05317400 & -1.93358200 & 2.79518900 \\ \mathrm{C} & 2.95206200 & -1.13560300 & 3.45099200 \\ \mathrm{H} & 2.76374400 & -1.26857700 & 4.51652200 \\ \mathrm{H} & 3.52385900 & -1.99203500 & 3.09909800 \\ \mathrm{H} & 3.55812300 & -0.24065200 & 3.31970600 \\ \mathrm{C} & -2.77709500 & 2.41717000 & 2.28875500 \\ \mathrm{H} & -2.95026400 & 1.86976000 & 1.36122200 \\ \mathrm{H} & -2.98279800 & 3.46799100 & 2.08029300 \\ \mathrm{C} & -3.64913200 & 1.90585700 & 3.42344400 \\ \mathrm{H} & -3.38178100 & 0.87100200 & 3.64497100 \\ \mathrm{H} & -3.43735900 & 2.48503800 & 4.32315900 \\ \mathrm{C} & -5.12821400 & 1.99736000 & 3.06643900 \\ \mathrm{H} & -5.75090900 & 1.63349600 & 3.88208700 \\ \mathrm{H} & -5.41591700 & 3.02849700 & 2.85835300 \\ \mathrm{H} & -5.35294900 & 1.40277100 & 2.18053200 \\ \mathrm{~S} & -0.98702500 & 2.38534900 & 2.63424800 \\ \mathrm{Cl} & 2.53320600 & 2.32569600 & 2.40678700\end{array}$

\section{$\underline{\text { DPTS } \bullet C l(P C 1)}$}




$\begin{array}{lrrr}\mathrm{O} & -0.49100600 & -1.32626000 & -1.12224200 \\ \mathrm{~S} & -0.72844400 & -1.66802500 & 0.32364000 \\ \mathrm{C} & 0.71442500 & -2.41335900 & 0.94257900 \\ \mathrm{H} & 0.60840400 & -2.83132000 & 1.93508200 \\ \mathrm{H} & 0.55668300 & -0.13113500 & -1.53944600 \\ \mathrm{C} & 2.01749700 & -2.30771700 & 0.24386000 \\ \mathrm{H} & 1.84707100 & -2.06815900 & -0.80580900 \\ \mathrm{H} & 2.51595100 & -3.27847300 & 0.28544200 \\ \mathrm{C} & 2.92035700 & -1.23733500 & 0.87978100 \\ \mathrm{H} & 3.09811900 & -1.45310800 & 1.93312900 \\ \mathrm{H} & 3.87877000 & -1.21086200 & 0.36366400 \\ \mathrm{H} & 2.45952300 & -0.25507600 & 0.79447000 \\ \mathrm{C} & -1.83208000 & 1.07718100 & 0.54537900 \\ \mathrm{H} & -1.56234600 & 1.07397400 & -0.51138400 \\ \mathrm{H} & -1.71055000 & 2.09954500 & 0.90787800 \\ \mathrm{C} & -3.24953700 & 0.57912400 & 0.77213100 \\ \mathrm{H} & -3.32998300 & -0.45638300 & 0.43558300 \\ \mathrm{H} & -3.46457300 & 0.58110700 & 1.84138100 \\ \mathrm{C} & -4.25745600 & 1.44194500 & 0.02119700 \\ \mathrm{H} & -5.27349400 & 1.08393000 & 0.17927300 \\ \mathrm{H} & -4.20939400 & 2.47832300 & 0.35727900 \\ \mathrm{H} & -4.05801200 & 1.42815500 & -1.05043800 \\ \mathrm{~S} & -0.53933200 & 0.15672900 & 1.43743200 \\ \mathrm{Cl} & 1.39586600 & 0.85875900 & -1.82381600\end{array}$

\section{$\underline{\mathrm{CH}}_{3} \underline{\mathrm{CH}}_{2}{\underline{\mathrm{C}} \cdot \mathbf{H}-\mathrm{S}(\mathrm{O})-\mathrm{S}-\mathrm{CH}_{2}}_{2}^{\mathrm{CH}_{2}} \underline{\mathrm{CH}}_{3}(\mathbf{P 1})$}

$\begin{array}{llll}\mathrm{O} & 0.15527200 & -0.14181400 & 0.21485100 \\ \mathrm{~S} & 0.10094000 & -0.36179200 & 1.68369400 \\ \mathrm{C} & 1.72544500 & -0.63430900 & 2.24033300 \\ \mathrm{H} & 1.82819000 & -0.83869400 & 3.29655800 \\ \mathrm{C} & 2.86520600 & -0.45348800 & 1.30819800 \\ \mathrm{H} & 2.60052400 & -0.87434400 & 0.33692100 \\ \mathrm{H} & 3.72857400 & -0.99705800 & 1.69090200 \\ \mathrm{C} & 3.20808100 & 1.03323400 & 1.11726700 \\ \mathrm{H} & 3.46888900 & 1.49864000 & 2.06732900 \\ \mathrm{H} & 4.05080700 & 1.13882600 & 0.43532700 \\ \mathrm{H} & 2.35252000 & 1.56001900 & 0.69735400 \\ \mathrm{C} & -1.92292400 & 1.70846900 & 2.32348300 \\ \mathrm{H} & -2.07166000 & 1.47677800 & 1.26750200 \\ \mathrm{H} & -2.12318600 & 2.77356700 & 2.44806000 \\ \mathrm{C} & -2.82771500 & 0.87569700 & 3.21695000 \\ \mathrm{H} & -2.56117100 & -0.17781300 & 3.11570200 \\ \mathrm{H} & -2.64626900 & 1.14663600 & 4.25790700 \\ \mathrm{C} & -4.29605700 & 1.07889700 & 2.86140400 \\ \mathrm{H} & -4.94198800 & 0.48335500 & 3.50478800 \\ \mathrm{H} & -4.58324700 & 2.12522200 & 2.97298200\end{array}$




\section{$\underline{\mathrm{HCl}}$}

$\begin{array}{llll}\mathrm{Cl} & 0.00000000 & 0.00000000 & 0.00537800\end{array}$

$\begin{array}{llll}\mathrm{H} & 0.00000000 & 0.00000000 & 1.28462200\end{array}$

\section{DPTS •Cl (RC2)}

$\begin{array}{lrrr}\mathrm{O} & -0.28322100 & -1.12129300 & -1.86960200 \\ \mathrm{~S} & -0.04074300 & -0.43433700 & -0.59205700 \\ \mathrm{C} & 1.74193800 & -0.40668100 & -0.27153100 \\ \mathrm{H} & 1.84364800 & 0.02174300 & 0.72559600 \\ \mathrm{H} & 2.16703300 & 0.26449100 & -1.01883600 \\ \mathrm{C} & 2.32269200 & -1.80834500 & -0.37288100 \\ \mathrm{H} & 2.10514500 & -2.20926900 & -1.36248600 \\ \mathrm{H} & 1.83453900 & -2.45220100 & 0.35751000 \\ \mathrm{C} & 3.82781800 & -1.77141500 & -0.13059400 \\ \mathrm{H} & 4.05508800 & -1.38613300 & 0.86353300 \\ \mathrm{H} & 4.25035900 & -2.77152600 & -0.20754700 \\ \mathrm{H} & 4.32950800 & -1.13696600 & -0.86187000 \\ \mathrm{C} & -2.12005600 & 1.55987700 & -1.09368400 \\ \mathrm{H} & -2.26378800 & 0.82181800 & -1.88381000 \\ \mathrm{H} & -2.35802200 & 2.53809100 & -1.51354900 \\ \mathrm{C} & -2.96605400 & 1.25955200 & 0.13306600 \\ \mathrm{H} & -2.65460900 & 0.30791700 & 0.56717600 \\ \mathrm{H} & -2.78121000 & 2.02363700 & 0.88908900 \\ \mathrm{C} & -4.44598500 & 1.21229200 & -0.22864700 \\ \mathrm{H} & -5.05249600 & 1.00104200 & 0.65024100 \\ \mathrm{H} & -4.77918600 & 2.16282400 & -0.64749400 \\ \mathrm{H} & -4.64172700 & 0.43327200 & -0.96586500 \\ \mathrm{~S} & -0.33023000 & 1.65357100 & -0.75694000 \\ \mathrm{Cl} & -0.63408800 & -1.66445200 & 1.49083700\end{array}$

\section{DPTS••Cl (TS1b)}

$\begin{array}{lrrr}\mathrm{O} & -0.48919000 & 0.24060500 & -0.60581600 \\ \mathrm{~S} & -0.49591600 & 0.30778300 & 0.88281000 \\ \mathrm{C} & 1.25255300 & 0.25517900 & 1.32631800 \\ \mathrm{H} & 1.20933500 & 0.45734700 & 2.75671300 \\ \mathrm{H} & 1.80026500 & 1.12280400 & 0.95992000 \\ \mathrm{C} & 1.89046500 & -1.08791400 & 1.17127200 \\ \mathrm{H} & 1.78268400 & -1.37937200 & 0.11943100 \\ \mathrm{H} & 1.33508800 & -1.82363700 & 1.75768300 \\ \mathrm{C} & 3.36292700 & -1.07578600 & 1.56650100 \\ \mathrm{H} & 3.47680100 & -0.80080300 & 2.61428100 \\ \mathrm{H} & 3.80401000 & -2.05960800 & 1.41975600\end{array}$




$\begin{array}{lrrr}\mathrm{H} & 3.91935100 & -0.36070500 & 0.96092800 \\ \mathrm{C} & -2.41723400 & 2.51047400 & 0.81941400 \\ \mathrm{H} & -2.41182400 & 2.15190500 & -0.21082800 \\ \mathrm{H} & -2.56953800 & 3.58986500 & 0.78668400 \\ \mathrm{C} & -3.49256600 & 1.83194700 & 1.65276600 \\ \mathrm{H} & -3.28574700 & 0.76179200 & 1.71114000 \\ \mathrm{H} & -3.44845600 & 2.21470000 & 2.67290300 \\ \mathrm{C} & -4.87539500 & 2.06120100 & 1.05388700 \\ \mathrm{H} & -5.64547600 & 1.57455100 & 1.65018900 \\ \mathrm{H} & -5.10867300 & 3.12563200 & 1.00776400 \\ \mathrm{H} & -4.93200600 & 1.66239600 & 0.04068500 \\ \mathrm{~S} & -0.72708700 & 2.32394500 & 1.47167700 \\ \mathrm{Cl} & 1.25252900 & 0.45947500 & 4.19089100\end{array}$

\section{$\underline{\text { DPTS ••Cl (PC2) }}$}

$\begin{array}{lccc}\mathrm{O} & -0.14483400 & -0.01060600 & -3.27898800 \\ \mathrm{~S} & -0.00743500 & -0.57911800 & -1.91880800 \\ \mathrm{C} & 1.61801500 & -1.12881000 & -1.69983600 \\ \mathrm{H} & 0.08922500 & -0.17082300 & 1.48535400 \\ \mathrm{H} & 2.29842700 & -0.87368100 & -2.50211300 \\ \mathrm{C} & 2.01926000 & -1.86268300 & -0.47345300 \\ \mathrm{H} & 2.45949600 & -2.82180100 & -0.76045500 \\ \mathrm{H} & 1.13756200 & -2.08922700 & 0.12959400 \\ \mathrm{C} & 3.03267100 & -1.07687800 & 0.36838300 \\ \mathrm{H} & 2.60938400 & -0.12783100 & 0.69577800 \\ \mathrm{H} & 3.30832900 & -1.64789000 & 1.25330100 \\ \mathrm{H} & 3.93396300 & -0.86814700 & -0.20707100 \\ \mathrm{C} & -1.61378400 & 1.56941900 & -0.63127100 \\ \mathrm{H} & -1.87302200 & 1.56706000 & -1.69161500 \\ \mathrm{H} & -1.63652700 & 2.60750100 & -0.29777500 \\ \mathrm{C} & -2.56012100 & 0.70711900 & 0.18797200 \\ \mathrm{H} & -2.44570800 & -0.33778400 & -0.10776200 \\ \mathrm{H} & -2.28076800 & 0.76547500 & 1.24118100 \\ \mathrm{C} & -4.00703100 & 1.14825000 & 0.00008600 \\ \mathrm{H} & -4.68251500 & 0.53445200 & 0.59359700 \\ \mathrm{H} & -4.14063200 & 2.18670600 & 0.30550600 \\ \mathrm{H} & -4.30648600 & 1.06603500 & -1.04511500 \\ \mathrm{~S} & 0.14028600 & 1.08430000 & -0.51477400 \\ \mathrm{Cl} & 0.06132500 & -0.92261200 & 2.54591900\end{array}$

\section{$\underline{\text { DPTS・・Cl (RC3) }}$}

$\begin{array}{llll}\mathrm{O} & -0.80956500 & -0.88900700 & -0.25860700\end{array}$ 


$\begin{array}{lrrr}\mathrm{S} & -0.36456200 & -0.29505300 & 1.06064400 \\ \mathrm{C} & 1.44814500 & -0.35036000 & 1.06909900 \\ \mathrm{H} & 1.75432500 & 0.09917500 & 2.01572100 \\ \mathrm{H} & 1.77499600 & 0.26601000 & 0.23268000 \\ \mathrm{C} & 1.91089600 & -1.79128500 & 0.92160400 \\ \mathrm{H} & 1.48937000 & -2.19308600 & -0.00015500 \\ \mathrm{H} & 1.52921000 & -2.39334900 & 1.74857400 \\ \mathrm{C} & 3.43310500 & -1.85870800 & 0.87511800 \\ \mathrm{H} & 3.87478000 & -1.45749500 & 1.78779400 \\ \mathrm{H} & 3.76939300 & -2.88769200 & 0.76123000 \\ \mathrm{H} & 3.81562700 & -1.28358900 & 0.03198600 \\ \mathrm{C} & -2.16389200 & 1.82323800 & 0.13275800 \\ \mathrm{H} & -2.07211900 & 1.24013400 & -0.78387700 \\ \mathrm{H} & -2.29291000 & 2.86966800 & -0.14815000 \\ \mathrm{C} & -3.30945800 & 1.34060000 & 1.00599800 \\ \mathrm{H} & -3.13159500 & 0.30316400 & 1.29611400 \\ \mathrm{H} & -3.33764600 & 1.92814500 & 1.92441500 \\ \mathrm{C} & -4.63626600 & 1.44184400 & 0.26226700 \\ \mathrm{H} & -5.45956300 & 1.09141600 & 0.88261300 \\ \mathrm{H} & -4.84525500 & 2.47344700 & -0.02337300 \\ \mathrm{H} & -4.61753600 & 0.83917900 & -0.64576500 \\ \mathrm{~S} & -0.52792700 & 1.80499100 & 0.92889600 \\ \mathrm{Cl} & 0.38075500 & -0.01781900 & -2.11329400 \\ & & & \end{array}$

\section{$\underline{\text { DPTS } \bullet C l ~(T S 2 a) ~}$}

$\begin{array}{lrrl}\mathrm{O} & -0.21185900 & -0.31621400 & -0.00426200 \\ \mathrm{~S} & -0.07423900 & 0.04135000 & 1.42944900 \\ \mathrm{C} & 1.71248000 & 0.08162200 & 1.78968000 \\ \mathrm{H} & 1.81917900 & 0.40044900 & 2.83114500 \\ \mathrm{H} & 2.17305500 & 0.82817600 & 1.13904100 \\ \mathrm{C} & 2.30799200 & -1.28036200 & 1.57355200 \\ \mathrm{H} & 2.58261800 & -1.30692100 & 0.23166800 \\ \mathrm{H} & 1.61086200 & -2.11620300 & 1.60881300 \\ \mathrm{C} & 3.65473500 & -1.52125400 & 2.18584800 \\ \mathrm{H} & 3.56301800 & -1.57111900 & 3.27537700 \\ \mathrm{H} & 4.08398200 & -2.45741700 & 1.83558100 \\ \mathrm{H} & 4.34147400 & -0.71109600 & 1.94154900 \\ \mathrm{C} & -2.03622600 & 2.20192100 & 1.16898200 \\ \mathrm{H} & -2.12955900 & 1.64326300 & 0.23635100 \\ \mathrm{H} & -2.21295300 & 3.25249500 & 0.93650000 \\ \mathrm{C} & -3.00802000 & 1.70536600 & 2.22719300 \\ \mathrm{H} & -2.77285400 & 0.66920300 & 2.47707600 \\ \mathrm{H} & -2.87095700 & 2.28739700 & 3.13942300\end{array}$




$\begin{array}{lccc}\mathrm{C} & -4.44871500 & 1.80771900 & 1.73978300 \\ \mathrm{H} & -5.14533300 & 1.45529800 & 2.49880200 \\ \mathrm{H} & -4.70662500 & 2.84007200 & 1.50035700 \\ \mathrm{H} & -4.59865100 & 1.20896300 & 0.84107800 \\ \mathrm{~S} & -0.28386000 & 2.13945100 & 1.65887600 \\ \mathrm{Cl} & 3.18089700 & -1.21836200 & -1.11560300\end{array}$

\section{$\underline{\text { DPTS } \bullet C I ~(P C 3) ~}$}

$\begin{array}{lccc}\mathrm{O} & -0.03363300 & -0.89161800 & -0.53015100 \\ \mathrm{~S} & -0.18933800 & -0.31964200 & 0.85790700 \\ \mathrm{C} & 1.53765600 & -0.18301800 & 1.55083600 \\ \mathrm{H} & 1.40470400 & 0.33618800 & 2.49978700 \\ \mathrm{H} & 2.07304800 & 0.44133300 & 0.83376600 \\ \mathrm{C} & 2.11030300 & -1.52859200 & 1.68078900 \\ \mathrm{H} & 1.11808900 & -0.23701300 & -1.43829300 \\ \mathrm{H} & 1.87971900 & -2.09385100 & 2.57286100 \\ \mathrm{C} & 2.78634900 & -2.19202200 & 0.53886400 \\ \mathrm{H} & 3.39617400 & -1.48249900 & -0.02266400 \\ \mathrm{H} & 3.41158700 & -3.01785000 & 0.87329200 \\ \mathrm{H} & 2.05511300 & -2.59173800 & -0.17452700 \\ \mathrm{C} & -2.15062700 & 1.68368600 & -0.03341100 \\ \mathrm{H} & -2.10927900 & 0.99889600 & -0.88133500 \\ \mathrm{H} & -2.30526700 & 2.68561800 & -0.43573700 \\ \mathrm{C} & -3.25161200 & 1.31164200 & 0.94600600 \\ \mathrm{H} & -3.04132200 & 0.32911700 & 1.37210600 \\ \mathrm{H} & -3.24738600 & 2.02131300 & 1.77421300 \\ \mathrm{C} & -4.61307500 & 1.29973600 & 0.26032500 \\ \mathrm{H} & -5.40219200 & 1.03484400 & 0.96214100 \\ \mathrm{H} & -4.84850700 & 2.27986300 & -0.15598400 \\ \mathrm{H} & -4.63183300 & 0.57694000 & -0.55571300 \\ \mathrm{~S} & -0.47987700 & 1.75755700 & 0.69042400 \\ \mathrm{Cl} & 2.13979200 & 0.34788500 & -2.07809700\end{array}$

\section{$\underline{\mathrm{CH}}_{3} \underline{\mathrm{C}}^{\cdot} \mathrm{HCH}_{2}-\mathrm{S}(\mathrm{O})-\mathrm{S}-\mathrm{CH}_{2} \underline{\mathrm{CH}}_{2} \underline{\mathrm{CH}}_{3} \underline{\text { (P2) }}$}

$\begin{array}{llrc}\mathrm{O} & -0.18514400 & -0.04932900 & 0.27929500 \\ \mathrm{~S} & 0.03986500 & -0.05365100 & 1.75050800 \\ \mathrm{C} & 1.88537000 & 0.08208100 & 1.99380600 \\ \mathrm{H} & 2.02088600 & 0.24704800 & 3.06273000 \\ \mathrm{H} & 2.15694000 & 0.97207600 & 1.42476200 \\ \mathrm{C} & 2.52792400 & -1.13808000 & 1.49264800 \\ \mathrm{H} & 2.61078000 & -1.98290900 & 2.16160400 \\ \mathrm{C} & 2.81027300 & -1.31450100 & 0.04609200 \\ \mathrm{H} & 3.51398000 & -2.12630300 & -0.12828000\end{array}$




$\begin{array}{lrrr}\mathrm{H} & 1.89058800 & -1.53392500 & -0.50883000 \\ \mathrm{H} & 3.21423300 & -0.39751300 & -0.38764700 \\ \mathrm{C} & -2.08974100 & 1.95507600 & 2.00641200 \\ \mathrm{H} & -2.13978700 & 1.65265700 & 0.95933000 \\ \mathrm{H} & -2.35567900 & 3.01162600 & 2.05495100 \\ \mathrm{C} & -3.01279100 & 1.11928800 & 2.87830500 \\ \mathrm{H} & -2.69264200 & 0.07624100 & 2.84680600 \\ \mathrm{H} & -2.91851600 & 1.44549900 & 3.91486600 \\ \mathrm{C} & -4.45978600 & 1.23167500 & 2.41227900 \\ \mathrm{H} & -5.12088000 & 0.63234300 & 3.03644600 \\ \mathrm{H} & -4.80393700 & 2.26586400 & 2.45461800 \\ \mathrm{H} & -4.56425700 & 0.88678700 & 1.38322800 \\ \mathrm{~S} & -0.33654200 & 1.90679600 & 2.49371800\end{array}$

\section{$\underline{\text { DPTS••Cl (TS2b) }}$}

$\begin{array}{lrrr}\mathrm{O} & 0.20097800 & 0.03013600 & -0.19525400 \\ \mathrm{~S} & 0.01971300 & 0.11997900 & 1.27794500 \\ \mathrm{C} & 1.71539800 & 0.07380600 & 1.96818200 \\ \mathrm{H} & 1.64872800 & 0.14889900 & 3.05391000 \\ \mathrm{H} & 2.22921900 & 0.95300700 & 1.56517100 \\ \mathrm{C} & 2.40010000 & -1.17726900 & 1.52156800 \\ \mathrm{H} & 2.23315600 & -1.43305800 & 0.47721500 \\ \mathrm{H} & 1.63296800 & -2.17858200 & 2.12527100 \\ \mathrm{C} & 3.76325100 & -1.44702800 & 2.07851300 \\ \mathrm{H} & 3.76883600 & -1.35737000 & 3.16462500 \\ \mathrm{H} & 4.10972800 & -2.44185300 & 1.80682700 \\ \mathrm{H} & 4.47624000 & -0.71921600 & 1.67727300 \\ \mathrm{C} & -1.98661600 & 2.26531300 & 1.25675900 \\ \mathrm{H} & -2.01856300 & 1.86169800 & 0.24358300 \\ \mathrm{H} & -2.16905300 & 3.33773800 & 1.18112400 \\ \mathrm{C} & -3.00802000 & 1.59999400 & 2.16512400 \\ \mathrm{H} & -2.76391000 & 0.54161000 & 2.26998600 \\ \mathrm{H} & -2.93755800 & 2.03856300 & 3.16129400 \\ \mathrm{C} & -4.41964200 & 1.75703800 & 1.61173500 \\ \mathrm{H} & -5.15061800 & 1.28279200 & 2.26459400 \\ \mathrm{H} & -4.68697000 & 2.81023100 & 1.51673500 \\ \mathrm{H} & -4.50406600 & 1.30056000 & 0.62514900 \\ \mathrm{~S} & -0.26557400 & 2.14036800 & 1.84090900 \\ \mathrm{Cl} & 0.85786900 & -3.09804300 & 2.95734700\end{array}$

\section{$\underline{\text { DPTS・・Cl (RC4) }}$}

$\begin{array}{llll}\mathrm{O} & -0.51589000 & -0.48706900 & -2.15687300\end{array}$ 


$\begin{array}{lrrr}\mathrm{S} & -0.46081700 & -0.27245200 & -0.68473400 \\ \mathrm{C} & 1.30262200 & -0.25366500 & -0.23804900 \\ \mathrm{H} & 1.36321600 & 0.02303100 & 0.81601900 \\ \mathrm{H} & 1.77047200 & 0.51610600 & -0.85274600 \\ \mathrm{C} & 1.89828200 & -1.62728500 & -0.50522100 \\ \mathrm{H} & 1.73188900 & -1.88444000 & -1.55165200 \\ \mathrm{H} & 1.37657200 & -2.37399600 & 0.09704500 \\ \mathrm{C} & 3.38918700 & -1.64971300 & -0.19158200 \\ \mathrm{H} & 3.58745200 & -1.34676600 & 0.84245400 \\ \mathrm{H} & 3.80746500 & -2.64471400 & -0.33278500 \\ \mathrm{H} & 3.93344600 & -0.95511500 & -0.83214700 \\ \mathrm{C} & -2.45124200 & 1.87746500 & -0.84339800 \\ \mathrm{H} & -2.49420900 & 1.39618300 & -1.82179200 \\ \mathrm{H} & -2.63672500 & 2.94103900 & -0.99677900 \\ \mathrm{C} & -3.46127800 & 1.28156400 & 0.12403400 \\ \mathrm{H} & -3.21725900 & 0.23244300 & 0.29981700 \\ \mathrm{H} & -3.37646200 & 1.78892200 & 1.08591600 \\ \mathrm{C} & -4.88048700 & 1.40132900 & -0.41924300 \\ \mathrm{H} & -5.60367200 & 0.97521700 & 0.27448100 \\ \mathrm{H} & -5.14813100 & 2.44581700 & -0.58410200 \\ \mathrm{H} & -4.97791000 & 0.87769600 & -1.37065500 \\ \mathrm{~S} & -0.72293500 & 1.79412100 & -0.27842300 \\ \mathrm{Cl} & 2.25460800 & -1.95430900 & 2.89067800 \\ & & & \end{array}$

\section{$\underline{\text { DPTS } \bullet C l ~(T S 3 a) ~}$}

$\begin{array}{lrrr}\mathrm{O} & 0.09791300 & 0.42581700 & 0.87854000 \\ \mathrm{~S} & 0.02439100 & 0.57885000 & 2.35792700 \\ \mathrm{C} & 1.74833800 & 0.54985700 & 2.94436800 \\ \mathrm{H} & 1.73767300 & 0.70846700 & 4.02275000 \\ \mathrm{H} & 2.26270900 & 1.37302100 & 2.44755400 \\ \mathrm{C} & 2.35882900 & -0.79280000 & 2.56751200 \\ \mathrm{H} & 2.23208600 & -0.94166900 & 1.48724200 \\ \mathrm{H} & 1.82337700 & -1.60759100 & 3.05961600 \\ \mathrm{C} & 3.81315500 & -0.86422200 & 2.89445700 \\ \mathrm{H} & 3.83938900 & -0.76875400 & 4.34086800 \\ \mathrm{H} & 4.30642900 & -1.82410300 & 2.78903000 \\ \mathrm{H} & 4.42785000 & -0.00167800 & 2.65639000 \\ \mathrm{C} & -1.95303200 & 2.74384700 & 2.24168800 \\ \mathrm{H} & -1.98059900 & 2.30108400 & 1.24479100 \\ \mathrm{H} & -2.12196000 & 3.81468700 & 2.12371700 \\ \mathrm{C} & -2.99007500 & 2.12635400 & 3.16589100 \\ \mathrm{H} & -2.76157400 & 1.06903000 & 3.31032400 \\ \mathrm{H} & -2.92016400 & 2.59915600 & 4.14629700\end{array}$




$\begin{array}{llll}\mathrm{C} & -4.39573600 & 2.28233600 & 2.59723800 \\ \mathrm{H} & -5.13807500 & 1.84260000 & 3.26144800 \\ \mathrm{H} & -4.64735300 & 3.33501500 & 2.46233800 \\ \mathrm{H} & -4.47982200 & 1.79194600 & 1.62702100 \\ \mathrm{~S} & -0.23847000 & 2.62162200 & 2.84287700 \\ \mathrm{Cl} & 3.69623600 & -0.66866900 & 5.76440700\end{array}$

\section{DPTS••Cl (PC4)}

$\begin{array}{lccc}\mathrm{O} & -0.51707700 & -0.49412000 & -2.24179700 \\ \mathrm{~S} & -0.45655800 & -0.27767400 & -0.76994400 \\ \mathrm{C} & 1.31221700 & -0.26289700 & -0.33809900 \\ \mathrm{H} & 1.38747200 & -0.02784300 & 0.72394900 \\ \mathrm{H} & 1.77223200 & 0.52653200 & -0.93304700 \\ \mathrm{C} & 1.90463200 & -1.62620400 & -0.67293900 \\ \mathrm{H} & 1.67298900 & -1.85134800 & -1.72233700 \\ \mathrm{H} & 1.41494800 & -2.40436300 & -0.08065900 \\ \mathrm{C} & 3.37512400 & -1.66457400 & -0.45770700 \\ \mathrm{H} & 3.19792000 & -1.64292100 & 1.71045800 \\ \mathrm{H} & 3.89047800 & -2.61390600 & -0.43504400 \\ \mathrm{H} & 3.97025100 & -0.77858700 & -0.63029200 \\ \mathrm{C} & -2.44990900 & 1.87208700 & -0.86945500 \\ \mathrm{H} & -2.52447800 & 1.39370600 & -1.84730200 \\ \mathrm{H} & -2.63677500 & 2.93656200 & -1.01482700 \\ \mathrm{C} & -3.43085900 & 1.27703600 & 0.12787600 \\ \mathrm{H} & -3.18434400 & 0.22710400 & 0.29487500 \\ \mathrm{H} & -3.31542900 & 1.78281000 & 1.08732000 \\ \mathrm{C} & -4.86555800 & 1.40124000 & -0.37198300 \\ \mathrm{H} & -5.56805700 & 0.97483100 & 0.34240200 \\ \mathrm{H} & -5.13584500 & 2.44667400 & -0.52596800 \\ \mathrm{H} & -4.99305000 & 0.88017000 & -1.32125500 \\ \mathrm{~S} & -0.70482900 & 1.78582100 & -0.35753000 \\ \mathrm{Cl} & 2.66397200 & -1.64517100 & 2.89060900\end{array}$

\section{$\underline{\mathrm{C}}^{\cdot} \mathrm{H}_{2} \underline{\mathrm{CH}}_{2} \underline{\mathrm{CH}_{2}} \underline{\mathrm{C}}_{2} \mathrm{~S}(\mathrm{O})-\mathrm{S}-\mathrm{CH}_{2} \underline{\mathrm{CH}}_{2} \underline{\mathrm{CH}}_{3}{ }_{2}(\mathrm{P} 3)$}

$\begin{array}{lrrr}\mathrm{O} & -0.14774100 & -0.11890500 & 0.13235200 \\ \mathrm{~S} & -0.00465000 & -0.04912700 & 1.61219400 \\ \mathrm{C} & 1.78183900 & 0.04887400 & 1.94693500 \\ \mathrm{H} & 1.89987900 & 0.20586500 & 3.01975500 \\ \mathrm{H} & 2.16090700 & 0.91182000 & 1.40025100 \\ \mathrm{C} & 2.44263000 & -1.24405600 & 1.47818600 \\ \mathrm{H} & 2.24069200 & -1.37953000 & 0.41534700 \\ \mathrm{H} & 1.96501700 & -2.09044000 & 1.99220500\end{array}$




$\begin{array}{lrrr}\mathrm{C} & 3.90481800 & -1.23169100 & 1.74028600 \\ \mathrm{H} & 4.28763300 & -0.84597800 & 2.67383600 \\ \mathrm{H} & 4.59036000 & -1.73753700 & 1.07945200 \\ \mathrm{C} & -2.13423700 & 1.95892900 & 1.81859900 \\ \mathrm{H} & -2.22058500 & 1.57821300 & 0.79963100 \\ \mathrm{H} & -2.39685800 & 3.01698000 & 1.79250800 \\ \mathrm{C} & -3.02904100 & 1.19823500 & 2.78384400 \\ \mathrm{H} & -2.70752600 & 0.15626600 & 2.82928600 \\ \mathrm{H} & -2.90367200 & 1.60944800 & 3.78633400 \\ \mathrm{C} & -4.48990200 & 1.27332600 & 2.35504700 \\ \mathrm{H} & -5.13078200 & 0.72985200 & 3.04763800 \\ \mathrm{H} & -4.83358600 & 2.30794600 & 2.32077200 \\ \mathrm{H} & -4.62628600 & 0.84335600 & 1.36239300 \\ \mathrm{~S} & -0.36615900 & 1.94090900 & 2.25149000\end{array}$

\section{$\underline{\text { DPTS } \bullet C I ~(T S 3 b) ~}$}

$\begin{array}{lrrr}\mathrm{O} & 0.05988200 & 0.01833500 & -0.04472900 \\ \mathrm{~S} & 0.00710700 & 0.10279100 & 1.43867800 \\ \mathrm{C} & 1.74397200 & 0.08973200 & 2.00271700 \\ \mathrm{H} & 1.74169000 & 0.27325700 & 3.07714900 \\ \mathrm{H} & 2.24176900 & 0.90809400 & 1.48296800 \\ \mathrm{C} & 2.35812200 & -1.26574900 & 1.64273700 \\ \mathrm{H} & 2.23658000 & -1.43168400 & 0.57208800 \\ \mathrm{H} & 1.82722100 & -2.06147400 & 2.16770900 \\ \mathrm{C} & 3.80640400 & -1.27570400 & 2.01551900 \\ \mathrm{H} & 4.05685000 & -1.30249700 & 3.07125300 \\ \mathrm{H} & 4.23564900 & -2.60092600 & 1.65629700 \\ \mathrm{H} & 4.48701100 & -0.69679200 & 1.39982000 \\ \mathrm{C} & -1.96720200 & 2.26900500 & 1.32368000 \\ \mathrm{H} & -1.93254600 & 1.88787100 & 0.30193200 \\ \mathrm{H} & -2.14139500 & 3.34377800 & 1.26356400 \\ \mathrm{C} & -3.04647700 & 1.58515100 & 2.14722100 \\ \mathrm{H} & -2.81616300 & 0.52182000 & 2.23396300 \\ \mathrm{H} & -3.03574700 & 1.99246100 & 3.15898200 \\ \mathrm{C} & -4.41999700 & 1.76877200 & 1.51212700 \\ \mathrm{H} & -5.19299500 & 1.27867400 & 2.10188900 \\ \mathrm{H} & -4.67581500 & 2.82623100 & 1.43511700 \\ \mathrm{H} & -4.44375500 & 1.34522400 & 0.50775100 \\ \mathrm{~S} & -0.28616600 & 2.12423200 & 2.00902800 \\ \mathrm{Cl} & 4.55059600 & -3.95062700 & 1.28908900\end{array}$

\section{$\underline{\text { DPTS } \bullet \cdot C l ~(T S 3 c)}$}




$\begin{array}{lrrr}\mathrm{O} & -0.03082500 & 0.51497100 & 1.45930300 \\ \mathrm{~S} & -0.58223300 & 0.86024100 & 2.79688200 \\ \mathrm{C} & 0.84831600 & 1.00097100 & 3.91652000 \\ \mathrm{H} & 0.46078600 & 1.28728300 & 4.89492300 \\ \mathrm{H} & 1.49713200 & 1.78553400 & 3.52696600 \\ \mathrm{C} & 1.56624000 & -0.34128100 & 3.94594100 \\ \mathrm{H} & 1.91096000 & -0.58747300 & 2.94090400 \\ \mathrm{H} & 0.86014300 & -1.13318600 & 4.23278700 \\ \mathrm{C} & 2.70308800 & -0.35990600 & 4.91458700 \\ \mathrm{H} & 2.53729100 & 0.06426500 & 5.90019400 \\ \mathrm{H} & 3.36866500 & -1.21561300 & 4.89007900 \\ \mathrm{H} & 3.62139800 & 0.63901700 & 4.40883000 \\ \mathrm{C} & -2.41253200 & 2.88169200 & 1.71655800 \\ \mathrm{H} & -2.09092900 & 2.31463900 & 0.84155100 \\ \mathrm{H} & -2.53946600 & 3.91759800 & 1.40053500 \\ \mathrm{C} & -3.69961000 & 2.32998100 & 2.30804200 \\ \mathrm{H} & -3.52644900 & 1.31333700 & 2.66528800 \\ \mathrm{H} & -3.97602500 & 2.92599700 & 3.17874800 \\ \mathrm{C} & -4.82414700 & 2.33546300 & 1.27894300 \\ \mathrm{H} & -5.74680200 & 1.94326200 & 1.70386800 \\ \mathrm{H} & -5.02178300 & 3.34729600 & 0.92291400 \\ \mathrm{H} & -4.56420400 & 1.72274300 & 0.41533600 \\ \mathrm{~S} & -1.00767700 & 2.93279400 & 2.87360300 \\ \mathrm{Cl} & 4.44478900 & 1.66278500 & 3.83784300\end{array}$

\section{DPTS••Cl (TS4a)}

$\begin{array}{lrrr}\mathrm{O} & -0.38937000 & -0.30314800 & 0.38225500 \\ \mathrm{~S} & -0.09407200 & 0.03996600 & 1.79794800 \\ \mathrm{C} & 1.71609100 & 0.08364400 & 1.95458600 \\ \mathrm{H} & 1.94349000 & 0.52054500 & 2.92894400 \\ \mathrm{H} & 2.07584600 & 0.74032000 & 1.16230900 \\ \mathrm{C} & 2.26357700 & -1.32905500 & 1.81380000 \\ \mathrm{H} & 1.92729600 & -1.74004200 & 0.86136500 \\ \mathrm{H} & 1.84987300 & -1.96169200 & 2.60176600 \\ \mathrm{C} & 3.78625400 & -1.33560300 & 1.88349100 \\ \mathrm{H} & 4.13722700 & -0.93651900 & 2.83561200 \\ \mathrm{H} & 4.17499500 & -2.34701700 & 1.77810200 \\ \mathrm{H} & 4.21257500 & -0.72621800 & 1.08651600 \\ \mathrm{C} & -1.96358300 & 2.27241700 & 1.35319400 \\ \mathrm{H} & -1.90080200 & 1.92779800 & 0.28475200 \\ \mathrm{H} & -2.18896100 & 3.33816900 & 1.32089400 \\ \mathrm{C} & -3.04648200 & 1.48671800 & 2.07407100 \\ \mathrm{H} & -2.80681400 & 0.42298100 & 2.04551100\end{array}$




$\begin{array}{llll}\mathrm{H} & -3.04746800 & 1.78820300 & 3.12344100 \\ \mathrm{C} & -4.41344800 & 1.72396200 & 1.44539400 \\ \mathrm{H} & -5.18494600 & 1.17650300 & 1.98454900 \\ \mathrm{H} & -4.67315800 & 2.78300900 & 1.46375000 \\ \mathrm{H} & -4.42437100 & 1.39533400 & 0.40676600 \\ \mathrm{~S} & -0.29313000 & 2.16425800 & 2.00494300 \\ \mathrm{Cl} & -2.47585100 & 2.30104000 & -1.58098400\end{array}$

\section{DPTS••Cl (RC5)}

$\begin{array}{lccc}\mathrm{O} & 0.23008600 & -0.68353500 & -2.14557700 \\ \mathrm{~S} & 0.23755000 & -0.67252700 & -0.66179300 \\ \mathrm{C} & 1.97971600 & -0.77377100 & -0.15727500 \\ \mathrm{H} & 2.01276100 & -0.53735800 & 0.90640700 \\ \mathrm{H} & 2.50850800 & -0.01476000 & -0.73490800 \\ \mathrm{C} & 2.49476100 & -2.17436600 & -0.45929400 \\ \mathrm{H} & 2.35154900 & -2.38544100 & -1.51958800 \\ \mathrm{H} & 1.90700100 & -2.90495700 & 0.09997400 \\ \mathrm{C} & 3.96861100 & -2.29942900 & -0.09060900 \\ \mathrm{H} & 4.12347300 & -2.10275400 & 0.97026700 \\ \mathrm{H} & 4.33783000 & -3.30047800 & -0.30661000 \\ \mathrm{H} & 4.57114600 & -1.58850500 & -0.65624100 \\ \mathrm{C} & -1.63426500 & 1.62405000 & -0.71785700 \\ \mathrm{H} & -1.59168000 & 1.23754800 & -1.73949400 \\ \mathrm{H} & -1.76796000 & 2.70437600 & -0.77196900 \\ \mathrm{C} & -2.74379200 & 0.96504800 & 0.08825200 \\ \mathrm{H} & -2.53514800 & -0.10057100 & 0.19185000 \\ \mathrm{H} & -2.74777100 & 1.37525300 & 1.09704000 \\ \mathrm{C} & -4.09006900 & 1.17953800 & -0.59294800 \\ \mathrm{H} & -4.88993800 & 0.71541800 & -0.01866500 \\ \mathrm{H} & -4.31756400 & 2.24218400 & -0.68608700 \\ \mathrm{H} & -4.09778900 & 0.74575700 & -1.59330500 \\ \mathrm{~S} & 0.04073700 & 1.40392200 & -0.05254500 \\ \mathrm{Cl} & -0.25357700 & 0.43368400 & 2.31889500\end{array}$

\section{DPTS••Cl (PC5)}

$\begin{array}{lccc}\mathrm{O} & -0.39102100 & -1.34690400 & -0.03005300 \\ \mathrm{~S} & 0.21636500 & -0.84946000 & 1.26067600 \\ \mathrm{C} & 1.98849900 & -0.61389700 & 0.91591600 \\ \mathrm{H} & 2.43189800 & -0.28220000 & 1.85536900 \\ \mathrm{H} & 2.07321900 & 0.17489000 & 0.16744500 \\ \mathrm{C} & 2.59412800 & -1.91477400 & 0.40982000 \\ \mathrm{H} & 2.02525700 & -2.25333300 & -0.45603000\end{array}$




$\begin{array}{lrrr}\mathrm{H} & 2.51089600 & -2.68585500 & 1.17820200 \\ \mathrm{C} & 4.05231700 & -1.70304500 & 0.01975400 \\ \mathrm{H} & 4.64194900 & -1.34999500 & 0.86640600 \\ \mathrm{H} & 4.49453100 & -2.63180600 & -0.33623200 \\ \mathrm{H} & 4.12697000 & -0.96535500 & -0.77934800 \\ \mathrm{C} & -1.79082000 & 1.26756400 & 1.97399600 \\ \mathrm{H} & 0.20752200 & -0.71560300 & -1.35066300 \\ \mathrm{H} & -2.00309700 & 1.93029000 & 2.79986100 \\ \mathrm{C} & -2.86639000 & 0.48724500 & 1.29826200 \\ \mathrm{H} & -2.65379100 & -0.58484300 & 1.37998400 \\ \mathrm{H} & -3.80303700 & 0.66264600 & 1.82712400 \\ \mathrm{C} & -3.01911000 & 0.83377100 & -0.18728300 \\ \mathrm{H} & -3.82123200 & 0.24515200 & -0.62923400 \\ \mathrm{H} & -3.25160700 & 1.89051600 & -0.31409300 \\ \mathrm{H} & -2.09893700 & 0.61529200 & -0.72688900 \\ \mathrm{~S} & -0.16155900 & 1.26036400 & 1.44408700 \\ \mathrm{Cl} & 0.84287600 & -0.13899200 & -2.38671400\end{array}$

\section{$\mathrm{CH}_{3} \underline{\mathrm{CH}}_{2} \underline{\mathrm{CH}}_{2}-\mathrm{S}(\mathrm{O})-\mathrm{S}-\mathrm{C}^{\cdot} \mathrm{HCH}_{2} \underline{\mathrm{CH}}_{3} \underline{(\mathrm{P} 4)}$}

$\begin{array}{lrrr}\mathrm{O} & 0.01488300 & -0.00521600 & -0.01302100 \\ \mathrm{~S} & 0.00096100 & -0.01909500 & 1.47316700 \\ \mathrm{C} & 1.74422900 & -0.01417100 & 1.99752500 \\ \mathrm{H} & 1.75095400 & 0.10048900 & 3.08238700 \\ \mathrm{H} & 2.20943900 & 0.85610500 & 1.53269300 \\ \mathrm{C} & 2.40669200 & -1.30796900 & 1.54822200 \\ \mathrm{H} & 2.28381100 & -1.40577300 & 0.46929500 \\ \mathrm{H} & 1.89678100 & -2.15727300 & 2.00775300 \\ \mathrm{C} & 3.88567200 & -1.32425200 & 1.91655100 \\ \mathrm{H} & 4.02303300 & -1.23726900 & 2.99480900 \\ \mathrm{H} & 4.35713600 & -2.24993500 & 1.59049100 \\ \mathrm{H} & 4.41121800 & -0.49427000 & 1.44383300 \\ \mathrm{C} & -1.96534600 & 2.22347700 & 1.80336200 \\ \mathrm{H} & -2.19991100 & 3.14397800 & 1.28653900 \\ \mathrm{C} & -3.01215900 & 1.18072900 & 1.98337900 \\ \mathrm{H} & -2.90524800 & 0.41843800 & 1.19997100 \\ \mathrm{H} & -2.84591500 & 0.65908100 & 2.92956300 \\ \mathrm{C} & -4.42011800 & 1.76512800 & 1.93042300 \\ \mathrm{H} & -5.17003900 & 0.98379500 & 2.04122500 \\ \mathrm{H} & -4.56575300 & 2.49501800 & 2.72625600 \\ \mathrm{H} & -4.59298700 & 2.26515600 & 0.97744100 \\ \mathrm{~S} & -0.32089200 & 2.00199500 & 2.22017100\end{array}$




\section{$\underline{\text { DPTS } \bullet C I ~(T S 4 b) ~}$}

$\begin{array}{lrrr}\mathrm{O} & 0.95128500 & 1.07833200 & -0.19732300 \\ \mathrm{~S} & 0.33757200 & 0.24466200 & 0.85832500 \\ \mathrm{C} & 1.69524600 & -0.20398800 & 1.98763000 \\ \mathrm{H} & 1.25321800 & -0.75297400 & 2.81967900 \\ \mathrm{H} & 2.12808700 & 0.72995200 & 2.34894600 \\ \mathrm{C} & 2.71809000 & -1.03145700 & 1.22309000 \\ \mathrm{H} & 3.05690600 & -0.45735900 & 0.36084200 \\ \mathrm{H} & 2.24205000 & -1.93668800 & 0.83994700 \\ \mathrm{C} & 3.89852800 & -1.39861800 & 2.11478900 \\ \mathrm{H} & 3.57441400 & -1.98289100 & 2.97641200 \\ \mathrm{H} & 4.63035700 & -1.98726000 & 1.56441200 \\ \mathrm{H} & 4.39728700 & -0.50266500 & 2.48479200 \\ \mathrm{C} & -2.20122100 & 1.61960200 & 1.56347300 \\ \mathrm{H} & -2.44073700 & 0.77010700 & 0.92337600 \\ \mathrm{H} & -2.09651400 & 2.56515200 & 0.71211100 \\ \mathrm{C} & -3.31613400 & 2.06672700 & 2.48351600 \\ \mathrm{H} & -3.39571100 & 1.34389400 & 3.30024600 \\ \mathrm{H} & -3.04346800 & 3.02408800 & 2.93126600 \\ \mathrm{C} & -4.64619100 & 2.18123700 & 1.74931900 \\ \mathrm{H} & -5.43217400 & 2.49303200 & 2.43477700 \\ \mathrm{H} & -4.58052600 & 2.91164200 & 0.94482700 \\ \mathrm{H} & -4.93430400 & 1.22165800 & 1.31915200 \\ \mathrm{~S} & -0.62707800 & 1.47668000 & 2.33020600 \\ \mathrm{Cl} & -2.15775700 & 3.81297600 & -0.25140500\end{array}$

\section{$\underline{\text { DPTS } \bullet C I ~(T S 5 a) ~}$}

$\begin{array}{llrr}\mathrm{O} & 2.16611100 & 1.05412900 & -1.85954700 \\ \mathrm{~S} & 1.44582300 & 0.90130900 & -0.57019000 \\ \mathrm{C} & 2.73804700 & 0.62337500 & 0.68099000 \\ \mathrm{H} & 2.23448000 & 0.60367300 & 1.64688600 \\ \mathrm{H} & 3.41472800 & 1.47793200 & 0.63013800 \\ \mathrm{C} & 3.45629100 & -0.68051000 & 0.37031200 \\ \mathrm{H} & 3.85541200 & -0.63041600 & -0.64284200 \\ \mathrm{H} & 2.73628700 & -1.50171000 & 0.39361200 \\ \mathrm{C} & 4.57613000 & -0.93965500 & 1.37140200 \\ \mathrm{H} & 4.18636100 & -1.00402200 & 2.38762700 \\ \mathrm{H} & 5.08933400 & -1.87340300 & 1.14719000 \\ \mathrm{H} & 5.31269900 & -0.13615700 & 1.34486300 \\ \mathrm{C} & -0.90194400 & 2.69573400 & -0.15918200 \\ \mathrm{H} & -1.06070700 & 1.98736600 & -0.97247100 \\ \mathrm{H} & -1.21684300 & 3.67789900 & -0.52222000\end{array}$




$\begin{array}{llll}\mathrm{C} & -1.73326600 & 2.33518200 & 1.04792100 \\ \mathrm{H} & -1.27731400 & 1.29325700 & 1.44274400 \\ \mathrm{H} & -1.51393800 & 2.97328500 & 1.90305600 \\ \mathrm{C} & -3.21168000 & 2.11296500 & 0.78808400 \\ \mathrm{H} & -3.49085000 & 1.07541900 & 0.97211800 \\ \mathrm{H} & -3.82530600 & 2.73798800 & 1.43368400 \\ \mathrm{H} & -3.46059900 & 2.33742700 & -0.24929000 \\ \mathrm{~S} & 0.87860800 & 2.81676100 & 0.18388300 \\ \mathrm{Cl} & -0.69284400 & -0.13814600 & 2.04764600\end{array}$

\section{DPTS••CI (PC6)}

$\begin{array}{lccc}\mathrm{O} & 0.28038500 & -1.76666600 & -1.12737000 \\ \mathrm{~S} & 0.45750500 & -1.49072600 & 0.32509300 \\ \mathrm{C} & 2.25440300 & -1.35267400 & 0.58546600 \\ \mathrm{H} & 2.40571300 & -1.07591400 & 1.62907300 \\ \mathrm{H} & 2.60415000 & -0.54896800 & -0.06403800 \\ \mathrm{C} & 2.91222700 & -2.67845800 & 0.23462100 \\ \mathrm{H} & 2.64767000 & -2.93823800 & -0.79042900 \\ \mathrm{H} & 2.51288000 & -3.46499900 & 0.87850900 \\ \mathrm{C} & 4.42643900 & -2.59556900 & 0.38822700 \\ \mathrm{H} & 4.70485300 & -2.34633000 & 1.41261300 \\ \mathrm{H} & 4.89494600 & -3.54468700 & 0.13322800 \\ \mathrm{H} & 4.84051000 & -1.82922400 & -0.26737600 \\ \mathrm{C} & -1.72498300 & 0.45827700 & 0.74513800 \\ \mathrm{H} & -2.05641900 & 1.38154200 & 1.22570800 \\ \mathrm{H} & -2.00545200 & -0.36611800 & 1.41041800 \\ \mathrm{C} & -2.31228100 & 0.32313200 & -0.61371600 \\ \mathrm{H} & -1.27441600 & 2.36398000 & -1.18265200 \\ \mathrm{H} & -1.83457900 & -0.36077100 & -1.30242500 \\ \mathrm{C} & -3.70680600 & 0.77986500 & -0.85391000 \\ \mathrm{H} & -3.93911900 & 0.81197900 & -1.91675400 \\ \mathrm{H} & -4.43476800 & 0.10816900 & -0.38158500 \\ \mathrm{H} & -3.87303400 & 1.77406400 & -0.43236100 \\ \mathrm{~S} & 0.10154000 & 0.55351400 & 0.74520900 \\ \mathrm{Cl} & -1.35712400 & 3.61900800 & -0.89408100\end{array}$

\section{$\underline{\mathrm{CH}}_{3} \underline{\mathrm{CH}}_{2} \underline{\mathrm{CH}}_{2} \underline{-\mathrm{S}(\mathrm{O})-\mathrm{S}-\mathrm{CH}_{2}} \underline{\mathrm{C}}^{\cdot} \mathrm{HCH}_{3} \underline{\text { (P5) }}$}

$\begin{array}{lrrr}\mathrm{O} & -0.06509900 & -0.02715900 & 0.10215400 \\ \mathrm{~S} & -0.03322500 & -0.08420700 & 1.59155900 \\ \mathrm{C} & 1.72201100 & -0.04677400 & 2.06531400 \\ \mathrm{H} & 1.75891000 & 0.08396400 & 3.14809600 \\ \mathrm{H} & 2.15270300 & 0.82602100 & 1.57405600\end{array}$




$\begin{array}{lrrr}\mathrm{C} & 2.39179500 & -1.33682700 & 1.61659500 \\ \mathrm{H} & 2.24121200 & -1.45262000 & 0.54280400 \\ \mathrm{H} & 1.90877800 & -2.18707200 & 2.10252500 \\ \mathrm{C} & 3.88054200 & -1.32468700 & 1.94341500 \\ \mathrm{H} & 4.04583400 & -1.21920300 & 3.01611100 \\ \mathrm{H} & 4.35795100 & -2.24760200 & 1.61815300 \\ \mathrm{H} & 4.37940200 & -0.49361400 & 1.44448800 \\ \mathrm{C} & -2.09603800 & 2.02404500 & 1.50929000 \\ \mathrm{H} & -1.89323500 & 1.90000000 & 0.44653600 \\ \mathrm{H} & -2.34353600 & 3.06554200 & 1.71009500 \\ \mathrm{C} & -3.10894600 & 1.08581300 & 2.00457200 \\ \mathrm{H} & -3.57919000 & 1.30215800 & 2.95426100 \\ \mathrm{C} & -3.34758700 & -0.23617500 & 1.36658800 \\ \mathrm{H} & -2.99595900 & -1.05975000 & 1.99774600 \\ \mathrm{H} & -4.41425200 & -0.40827400 & 1.20138100 \\ \mathrm{H} & -2.83124400 & -0.31234800 & 0.40949800 \\ \mathrm{~S} & -0.43239600 & 1.85960200 & 2.34731600\end{array}$

\section{$\underline{\text { DPTS } \bullet C l ~(T S 5 b) ~}$}

$\begin{array}{lccc}\mathrm{O} & 0.04819300 & 0.15366500 & -0.04989100 \\ \mathrm{~S} & 0.01068200 & 0.08028100 & 1.43622300 \\ \mathrm{C} & 1.73829300 & 0.00087800 & 1.99641700 \\ \mathrm{H} & 1.72819100 & 0.11197900 & 3.08191200 \\ \mathrm{H} & 2.24572500 & 0.85251200 & 1.54222800 \\ \mathrm{C} & 2.34861100 & -1.32113400 & 1.55574900 \\ \mathrm{H} & 2.24350300 & -1.41099500 & 0.47414600 \\ \mathrm{H} & 1.79187500 & -2.14701800 & 2.00316200 \\ \mathrm{C} & 3.81772500 & -1.40492500 & 1.95321300 \\ \mathrm{H} & 3.93701200 & -1.32615000 & 3.03415200 \\ \mathrm{H} & 4.25264500 & -2.35056100 & 1.63411900 \\ \mathrm{H} & 4.38972100 & -0.59901000 & 1.49311200 \\ \mathrm{C} & -1.91864300 & 2.25865100 & 1.42943100 \\ \mathrm{H} & -1.82730400 & 1.93066600 & 0.38731400 \\ \mathrm{H} & -2.09950300 & 3.33429200 & 1.41226000 \\ \mathrm{C} & -3.04371400 & 1.54230500 & 2.11059600 \\ \mathrm{H} & -2.80363400 & 0.55421700 & 2.49806000 \\ \mathrm{H} & -3.12659900 & 2.22041400 & 3.32010000 \\ \mathrm{C} & -4.41189600 & 1.71688400 & 1.52861100 \\ \mathrm{H} & -5.17691100 & 1.30451700 & 2.18321000 \\ \mathrm{H} & -4.63027800 & 2.77073900 & 1.35584600 \\ \mathrm{H} & -4.47318800 & 1.19867900 & 0.56608300 \\ \mathrm{~S} & -0.29238700 & 2.04188100 & 2.20560900 \\ \mathrm{Cl} & -3.25956600 & 3.11570900 & 4.47297600\end{array}$




\section{DPTS••Cl (PC7)}

$\begin{array}{lccc}\mathrm{O} & 0.94130800 & -0.84921300 & -2.28737800 \\ \mathrm{~S} & 0.76068800 & -1.05820500 & -0.82518600 \\ \mathrm{C} & 2.42929600 & -1.13843500 & -0.10606300 \\ \mathrm{H} & 2.31134800 & -1.13511300 & 0.97869800 \\ \mathrm{H} & 2.94381600 & -0.23126600 & -0.42450900 \\ \mathrm{C} & 3.13200800 & -2.39096500 & -0.60755100 \\ \mathrm{H} & 3.13488000 & -2.37652000 & -1.69784700 \\ \mathrm{H} & 2.56839400 & -3.27386000 & -0.29905600 \\ \mathrm{C} & 4.55830700 & -2.46910900 & -0.07566800 \\ \mathrm{H} & 4.56999000 & -2.49346400 & 1.01441100 \\ \mathrm{H} & 5.05948200 & -3.36506700 & -0.43816600 \\ \mathrm{H} & 5.13926600 & -1.60473500 & -0.39776800 \\ \mathrm{C} & -1.26536200 & 1.04637500 & -0.80662100 \\ \mathrm{H} & -1.05090100 & 0.86619400 & -1.86673200 \\ \mathrm{H} & -1.49475300 & 2.10887000 & -0.70349700 \\ \mathrm{C} & -2.37862000 & 0.20710300 & -0.29533600 \\ \mathrm{H} & -2.16292100 & -0.82586300 & -0.05506900 \\ \mathrm{H} & -2.17323600 & 1.26335800 & 1.72108300 \\ \mathrm{C} & -3.78549000 & 0.62418900 & -0.53783000 \\ \mathrm{H} & -4.48673700 & 0.03210600 & 0.04731000 \\ \mathrm{H} & -3.92945500 & 1.67809400 & -0.28914100 \\ \mathrm{H} & -4.05826100 & 0.50623000 & -1.59385600 \\ \mathrm{~S} & 0.31386600 & 0.81490800 & 0.07960000 \\ \mathrm{Cl} & -2.28905600 & 2.38188100 & 2.35627100\end{array}$

\section{DPTS••Cl (TS6a)}

$\begin{array}{lrrr}\mathrm{O} & -0.05218000 & 0.04005800 & 0.01141700 \\ \mathrm{~S} & -0.02986700 & 0.09175700 & 1.49836300 \\ \mathrm{C} & 1.71901200 & 0.04802300 & 1.99284100 \\ \mathrm{H} & 1.75437900 & 0.25627900 & 3.06350200 \\ \mathrm{H} & 2.21282000 & 0.84993700 & 1.44340400 \\ \mathrm{C} & 2.30179400 & -1.31496200 & 1.65006000 \\ \mathrm{H} & 2.15424700 & -1.50005900 & 0.58564700 \\ \mathrm{H} & 1.75636000 & -2.09125700 & 2.19047100 \\ \mathrm{C} & 3.78451700 & -1.37942100 & 1.99741300 \\ \mathrm{H} & 3.94607700 & -1.20661100 & 3.06182700 \\ \mathrm{H} & 4.19943600 & -2.35443400 & 1.74764300 \\ \mathrm{H} & 4.34455900 & -0.62389300 & 1.44608800 \\ \mathrm{C} & -1.92591500 & 2.31031200 & 1.32710500 \\ \mathrm{H} & -1.84749000 & 1.93412700 & 0.30664200 \\ \mathrm{H} & -2.08868900 & 3.38614700 & 1.27561100\end{array}$




$\begin{array}{llll}\mathrm{C} & -3.05668300 & 1.61922300 & 2.09958300 \\ \mathrm{H} & -2.84003400 & 0.55333600 & 2.18012100 \\ \mathrm{H} & -3.09935500 & 2.02267000 & 3.11095400 \\ \mathrm{C} & -4.35764900 & 1.82598900 & 1.39256300 \\ \mathrm{H} & -5.30005300 & 1.13209200 & 2.22878800 \\ \mathrm{H} & -4.79996800 & 2.81672900 & 1.42577300 \\ \mathrm{H} & -4.51818400 & 1.28752700 & 0.46408700 \\ \mathrm{~S} & -0.29179700 & 2.11957800 & 2.09986900 \\ \mathrm{Cl} & -6.15728900 & 0.42032300 & 3.13369200\end{array}$

\section{$\underline{\text { DPTS } \bullet \text { Cl (PC8) }}$}

$\begin{array}{lccc}\mathrm{O} & 1.33197400 & -0.47781000 & -1.99281100 \\ \mathrm{~S} & 1.23411500 & -0.48395800 & -0.50781300 \\ \mathrm{C} & 2.93589500 & -0.60790900 & 0.12084900 \\ \mathrm{H} & 2.89123400 & -0.45232900 & 1.19993600 \\ \mathrm{H} & 3.49490000 & 0.20304200 & -0.34694600 \\ \mathrm{C} & 3.50639200 & -1.97037400 & -0.24338100 \\ \mathrm{H} & 3.43672000 & -2.10032300 & -1.32376300 \\ \mathrm{H} & 2.89969000 & -2.75410700 & 0.21477100 \\ \mathrm{C} & 4.95539400 & -2.09731900 & 0.21217800 \\ \mathrm{H} & 5.03902300 & -1.97840100 & 1.29285100 \\ \mathrm{H} & 5.36138400 & -3.07239300 & -0.05183900 \\ \mathrm{H} & 5.57715600 & -1.33525800 & -0.25805900 \\ \mathrm{C} & -0.60614500 & 1.78726200 & -0.67296300 \\ \mathrm{H} & -0.49338800 & 1.45449900 & -1.70509500 \\ \mathrm{H} & -0.73964700 & 2.86824300 & -0.68483400 \\ \mathrm{C} & -1.79345800 & 1.09761100 & 0.01455100 \\ \mathrm{H} & -1.59967700 & 0.02397100 & 0.05647600 \\ \mathrm{H} & -1.85845900 & 1.45500700 & 1.04295400 \\ \mathrm{C} & -3.05648800 & 1.36759500 & -0.72429800 \\ \mathrm{H} & -4.24889100 & 0.18116700 & 0.67220800 \\ \mathrm{H} & -3.59742200 & 2.28870200 & -0.55902900 \\ \mathrm{H} & -3.29035000 & 0.80887100 & -1.61935700 \\ \mathrm{~S} & 0.98817000 & 1.52131500 & 0.16176100 \\ \mathrm{Cl} & -4.69555400 & -0.53653200 & 1.65089000\end{array}$

\section{$\underline{\mathrm{CH}}_{3} \underline{\mathrm{CH}_{2}} \underline{\mathrm{CH}} \underline{2}-\underline{\mathrm{S}(\mathrm{O})-\mathrm{S}-\mathrm{CH}_{2}} \underline{\mathrm{CH}_{2}} \underline{\mathrm{C}^{\circ} \mathrm{H}_{2}} \underline{(\mathrm{P} 6)}$}

$\begin{array}{lrrr}\mathrm{O} & -0.02229900 & -0.01623000 & 0.01680700 \\ \mathrm{~S} & -0.00354600 & -0.01682100 & 1.50506000 \\ \mathrm{C} & 1.74837600 & -0.00730600 & 1.99564200 \\ \mathrm{H} & 1.77783100 & 0.13485700 & 3.07695300 \\ \mathrm{H} & 2.20232700 & 0.85022400 & 1.49786500\end{array}$




$\begin{array}{lrrr}\mathrm{C} & 2.39603700 & -1.31496500 & 1.56633100 \\ \mathrm{H} & 2.25166100 & -1.43933500 & 0.49274300 \\ \mathrm{H} & 1.89206800 & -2.15009600 & 2.05721900 \\ \mathrm{C} & 3.88191700 & -1.32870600 & 1.90577800 \\ \mathrm{H} & 4.04068300 & -1.21498200 & 2.97860900 \\ \mathrm{H} & 4.34323500 & -2.26444000 & 1.59430000 \\ \mathrm{H} & 4.40144500 & -0.51335800 & 1.40199600 \\ \mathrm{C} & -2.05251800 & 2.08262300 & 1.57017900 \\ \mathrm{H} & -2.04421900 & 1.74846600 & 0.53247100 \\ \mathrm{H} & -2.27474900 & 3.14902500 & 1.57171700 \\ \mathrm{C} & -3.08509500 & 1.30975300 & 2.40316300 \\ \mathrm{H} & -2.79765200 & 0.25644100 & 2.41931300 \\ \mathrm{H} & -3.04153900 & 1.67317200 & 3.43042300 \\ \mathrm{C} & -4.45074100 & 1.46704100 & 1.83927700 \\ \mathrm{H} & -5.08155600 & 2.28910000 & 2.14117400 \\ \mathrm{H} & -4.77093000 & 0.86422600 & 1.00315600 \\ \mathrm{~S} & -0.34695800 & 1.96176600 & 2.19712700\end{array}$

\section{DPTS••Cl (TS6b)}

$\begin{array}{lccc}\mathrm{O} & 0.33706200 & -0.49160900 & 0.61202500 \\ \mathrm{~S} & 0.44656300 & -0.34009300 & 2.08938200 \\ \mathrm{C} & 2.22162500 & -0.33254900 & 2.48000000 \\ \mathrm{H} & 2.31824800 & -0.04057200 & 3.52721300 \\ \mathrm{H} & 2.66940300 & 0.42947100 & 1.84166800 \\ \mathrm{C} & 2.80195300 & -1.71240500 & 2.20891600 \\ \mathrm{H} & 2.59024200 & -1.98280900 & 1.17391600 \\ \mathrm{H} & 2.30350500 & -2.44885700 & 2.84248600 \\ \mathrm{C} & 4.30457100 & -1.73491700 & 2.46392100 \\ \mathrm{H} & 4.53098200 & -1.47548700 & 3.49852900 \\ \mathrm{H} & 4.71717600 & -2.72282900 & 2.26622600 \\ \mathrm{H} & 4.81716100 & -1.02075700 & 1.81928400 \\ \mathrm{C} & -1.45223500 & 1.86533400 & 1.80373600 \\ \mathrm{H} & -1.36990600 & 1.47930900 & 0.78695900 \\ \mathrm{H} & -1.64011600 & 2.93732800 & 1.74486900 \\ \mathrm{C} & -2.56388800 & 1.16714700 & 2.57506100 \\ \mathrm{H} & -2.33113900 & 0.09762200 & 2.66491700 \\ \mathrm{H} & -2.61554900 & 1.55525500 & 3.59343600 \\ \mathrm{C} & -3.88781000 & 1.29767900 & 1.89688000 \\ \mathrm{H} & -4.76534800 & 0.93870400 & 2.42316000 \\ \mathrm{H} & -4.16344400 & 2.71796500 & 1.97659800 \\ \mathrm{H} & -3.91733600 & 1.17895700 & 0.81802600 \\ \mathrm{~S} & 0.18703200 & 1.71979400 & 2.56886400 \\ \mathrm{Cl} & -4.31599300 & 4.13426200 & 2.14777500\end{array}$




\section{DPTS••Cl (PC9)}

$\begin{array}{lccc}\mathrm{O} & 0.94418700 & -1.58609000 & -1.59692700 \\ \mathrm{~S} & 0.98558200 & -1.36009100 & -0.12595900 \\ \mathrm{C} & 2.74294600 & -1.27091500 & 0.33269500 \\ \mathrm{H} & 2.78716200 & -0.94561800 & 1.37338700 \\ \mathrm{H} & 3.18805900 & -0.51150700 & -0.31078600 \\ \mathrm{C} & 3.38534500 & -2.63416200 & 0.12522700 \\ \mathrm{H} & 3.22447600 & -2.94166300 & -0.90851000 \\ \mathrm{H} & 2.89047700 & -3.37129900 & 0.76087000 \\ \mathrm{C} & 4.87653000 & -2.59107900 & 0.43792200 \\ \mathrm{H} & 5.05246700 & -2.29452800 & 1.47238300 \\ \mathrm{H} & 5.33390500 & -3.56714900 & 0.28475700 \\ \mathrm{H} & 5.38650300 & -1.87545200 & -0.20723000 \\ \mathrm{C} & -1.02062300 & 0.74284900 & -0.48219000 \\ \mathrm{H} & -0.95257000 & 0.27337100 & -1.46454400 \\ \mathrm{H} & -1.23247700 & 1.80176500 & -0.63038000 \\ \mathrm{C} & -2.09652500 & 0.08864700 & 0.37622300 \\ \mathrm{H} & -1.81011400 & -0.95285100 & 0.57671800 \\ \mathrm{H} & -2.13633600 & 0.57779300 & 1.35224800 \\ \mathrm{C} & -3.42998300 & 0.11880700 & -0.28126700 \\ \mathrm{H} & -4.32176300 & -0.09265500 & 0.29111600 \\ \mathrm{H} & -3.68165700 & 2.24845200 & 0.05873400 \\ \mathrm{H} & -3.50293100 & 0.05303700 & -1.35818200 \\ \mathrm{~S} & 0.64088200 & 0.70516300 & 0.25036200 \\ \mathrm{Cl} & -3.48055500 & 3.44856800 & 0.50327900\end{array}$

\section{DPTS••Cl (TS6c)}

$\begin{array}{lrrr}\mathrm{O} & 1.61836900 & -0.12484700 & 1.69128100 \\ \mathrm{~S} & 0.79586500 & -0.44209800 & 2.88584300 \\ \mathrm{C} & 1.90094600 & -0.26210500 & 4.32212800 \\ \mathrm{H} & 1.29098400 & -0.39675000 & 5.21606200 \\ \mathrm{H} & 2.29058900 & 0.75631500 & 4.29062000 \\ \mathrm{C} & 3.01798200 & -1.28977900 & 4.22150400 \\ \mathrm{H} & 3.52838900 & -1.15678600 & 3.26746600 \\ \mathrm{H} & 2.58985100 & -2.29442300 & 4.21885700 \\ \mathrm{C} & 4.00283000 & -1.14396000 & 5.37555200 \\ \mathrm{H} & 3.50691100 & -1.28576600 & 6.33631000 \\ \mathrm{H} & 4.80301800 & -1.87815400 & 5.29792800 \\ \mathrm{H} & 4.45532400 & -0.15206400 & 5.37514000 \\ \mathrm{C} & -1.60326500 & 1.05766800 & 2.08797100 \\ \mathrm{H} & -1.07513100 & 0.77895800 & 1.17440300 \\ \mathrm{H} & -2.02112900 & 2.05222800 & 1.93253500\end{array}$




$\begin{array}{lccc}\mathrm{C} & -2.69718200 & 0.05503500 & 2.42736300 \\ \mathrm{H} & -2.25365700 & -0.91528600 & 2.66138300 \\ \mathrm{H} & -3.21606100 & 0.37972600 & 3.33758700 \\ \mathrm{C} & -3.69530600 & -0.09572200 & 1.32538000 \\ \mathrm{H} & -4.47936200 & -0.83440300 & 1.45051400 \\ \mathrm{H} & -3.98843100 & 0.79507500 & 0.77770400 \\ \mathrm{H} & -2.93656200 & -0.76154000 & 0.30011200 \\ \mathrm{~S} & -0.37249500 & 1.25470500 & 3.41100300 \\ \mathrm{Cl} & -2.03946800 & -1.45332000 & -0.58264800\end{array}$

\section{$\underline{\text { DPTS } \bullet C l ~(P C 10) ~}$}

$\begin{array}{lccc}\mathrm{O} & 1.55606000 & 0.19776600 & -0.78714900 \\ \mathrm{~S} & 0.76639800 & -0.12144700 & 0.43035800 \\ \mathrm{C} & 1.92794400 & 0.00536700 & 1.82767200 \\ \mathrm{H} & 1.34770300 & -0.13050200 & 2.74095500 \\ \mathrm{H} & 2.34221300 & 1.01411700 & 1.80015800 \\ \mathrm{C} & 3.01398300 & -1.04765200 & 1.66871400 \\ \mathrm{H} & 3.49310500 & -0.90975400 & 0.69925500 \\ \mathrm{H} & 2.56031900 & -2.04106100 & 1.66383900 \\ \mathrm{C} & 4.04311300 & -0.94842600 & 2.78858000 \\ \mathrm{H} & 3.57834000 & -1.09565600 & 3.76401000 \\ \mathrm{H} & 4.82109400 & -1.70076700 & 2.66929000 \\ \mathrm{H} & 4.52051300 & 0.03174800 & 2.78958800 \\ \mathrm{C} & -1.63195100 & 1.45090500 & -0.23741100 \\ \mathrm{H} & -1.14478700 & 1.22293700 & -1.18674100 \\ \mathrm{H} & -2.05878400 & 2.45046900 & -0.31996900 \\ \mathrm{C} & -2.70866100 & 0.42796200 & 0.10145300 \\ \mathrm{H} & -2.24483600 & -0.54932400 & 0.26489200 \\ \mathrm{H} & -3.16448800 & 0.70051400 & 1.06190500 \\ \mathrm{C} & -3.75023000 & 0.33203400 & -0.95565200 \\ \mathrm{H} & -4.48297400 & -0.46128500 & -0.92746900 \\ \mathrm{H} & -3.94705500 & 1.17109100 & -1.60836500 \\ \mathrm{H} & -2.33324400 & -0.95710400 & -2.26979700 \\ \mathrm{~S} & -0.33782200 & 1.59066400 & 1.03191900 \\ \mathrm{Cl} & -1.14239700 & -1.40980500 & -2.46327200\end{array}$

\section{DPTS••CI (TS7a)}

$\begin{array}{lrrr}\mathrm{O} & -0.90908100 & -1.15847400 & -1.69838600 \\ \mathrm{~S} & -0.87250500 & -1.06937500 & -0.21541700 \\ \mathrm{C} & 0.87164000 & -1.12490900 & 0.25325600 \\ \mathrm{H} & 0.90281000 & -0.93978300 & 1.32920000 \\ \mathrm{H} & 1.36140100 & -0.30539700 & -0.27378500\end{array}$




$\begin{array}{lrrr}\mathrm{C} & 1.47899500 & -2.47275100 & -0.11783700 \\ \mathrm{H} & 1.33637100 & -2.63396100 & -1.18657200 \\ \mathrm{H} & 0.94768700 & -3.27218000 & 0.40237200 \\ \mathrm{C} & 2.96240500 & -2.51680200 & 0.23002600 \\ \mathrm{H} & 3.11945500 & -2.36533100 & 1.29846100 \\ \mathrm{H} & 3.39661500 & -3.47778400 & -0.04129200 \\ \mathrm{H} & 3.50730500 & -1.73740300 & -0.30268600 \\ \mathrm{C} & -2.75959000 & 1.39869800 & -0.30685400 \\ \mathrm{H} & -2.65690400 & 0.81358400 & -1.22336600 \\ \mathrm{H} & -2.93038200 & 2.43624000 & -0.59032100 \\ \mathrm{C} & -3.86977600 & 0.86042400 & 0.58572600 \\ \mathrm{H} & -3.64092700 & -0.16984900 & 0.86376900 \\ \mathrm{H} & -3.90999300 & 1.43823800 & 1.51046400 \\ \mathrm{C} & -5.21455300 & 0.92450400 & -0.13102600 \\ \mathrm{H} & -6.01152300 & 0.53864000 & 0.50239000 \\ \mathrm{H} & -5.46399600 & 1.95125400 & -0.39951800 \\ \mathrm{H} & -5.19314300 & 0.33362200 & -1.04672000 \\ \mathrm{~S} & -1.15449700 & 1.33744200 & 0.52736800 \\ \mathrm{Cl} & 0.11481500 & 1.82683000 & -1.28606700\end{array}$

\section{$\underline{\text { DPTS } \bullet C l ~(P C 11) ~}$}

$\begin{array}{lrrr}\mathrm{O} & -1.15897400 & -1.94502900 & 0.14930700 \\ \mathrm{~S} & -0.39088600 & -1.88767600 & 1.44610300 \\ \mathrm{C} & 1.29706700 & -1.44524500 & 0.97876200 \\ \mathrm{H} & 1.83106600 & -1.28192100 & 1.91745000 \\ \mathrm{H} & 1.23882400 & -0.48970300 & 0.45437400 \\ \mathrm{C} & 1.95716300 & -2.51257000 & 0.11576500 \\ \mathrm{H} & 1.32085900 & -2.69926400 & -0.75003400 \\ \mathrm{H} & 2.01665100 & -3.44735500 & 0.67622600 \\ \mathrm{C} & 3.34675600 & -2.07607700 & -0.33272900 \\ \mathrm{H} & 3.99625900 & -1.89613300 & 0.52455900 \\ \mathrm{H} & 3.81376900 & -2.83784200 & -0.95518100 \\ \mathrm{H} & 3.29428200 & -1.15265100 & -0.91012700 \\ \mathrm{C} & -1.32694600 & 1.30997000 & 0.17733800 \\ \mathrm{H} & -0.85881900 & 0.47770900 & -0.34829500 \\ \mathrm{H} & -1.38793800 & 2.17227600 & -0.48476300 \\ \mathrm{C} & -2.70584300 & 0.90758500 & 0.68831700 \\ \mathrm{H} & -2.60192900 & 0.11748300 & 1.43462100 \\ \mathrm{H} & -3.17499500 & 1.76081500 & 1.18130400 \\ \mathrm{C} & -3.57482100 & 0.40794400 & -0.46035200 \\ \mathrm{H} & -4.56910500 & 0.14133600 & -0.10550200 \\ \mathrm{H} & -3.68449800 & 1.17507100 & -1.22772000 \\ \mathrm{H} & -3.12562100 & -0.47515700 & -0.91313400\end{array}$




$\begin{array}{lrrr}\mathrm{S} & -0.31026600 & 1.75670700 & 1.60757100 \\ \mathrm{Cl} & 1.40771800 & 2.32359200 & 0.65417000\end{array}$

$\begin{array}{lrrr}\mathbf{C H}_{3} & { }_{\mathbf{C H}} \\ \mathbf{C} & \mathbf{C H} \\ \mathrm{H} & -0.06261600 & -0.00424000 & -0.04047600 \\ \mathrm{SCl}(\mathbf{P} 7 \mathbf{b}) & & & \\ \mathrm{H} & -0.04632900 & 0.03859300 & 1.04867100 \\ \mathrm{C} & 0.97103700 & 0.07504100 & -0.38986600 \\ \mathrm{H} & -0.71202200 & -1.28151400 & -0.54439100 \\ \mathrm{H} & -1.76322000 & -1.28295800 & -0.25387600 \\ \mathrm{C} & -0.68390000 & -1.28791500 & -1.63468400 \\ \mathrm{H} & -0.01246100 & -2.51450000 & 0.01599600 \\ \mathrm{H} & -0.47984000 & -3.42843500 & -0.34704000 \\ \mathrm{H} & 1.03829200 & -2.53626500 & -0.27598700 \\ \mathrm{~S} & -0.05597100 & -2.52620000 & 1.10559100 \\ \mathrm{Cl} & -0.80691600 & 1.52216200 & -0.65884500 \\ & -2.62335700 & 1.42785600 & 0.27157400\end{array}$

\section{$\underline{\mathrm{CH}}_{3} \underline{\mathrm{CH}}_{2} \underline{\mathrm{CH}_{2}} \underline{\mathrm{S}^{*}(=\mathrm{O})(\mathrm{P} 7 \mathrm{a})}$}

$\begin{array}{lrrr}\mathrm{O} & 0.19145200 & 0.49571100 & 0.15213700 \\ \mathrm{~S} & -0.07137600 & -0.15242600 & 1.48464500 \\ \mathrm{C} & 1.51064000 & -0.08856400 & 2.36363600 \\ \mathrm{H} & 1.32253400 & -0.52577000 & 3.34647200 \\ \mathrm{H} & 1.75738600 & 0.96651900 & 2.49482100 \\ \mathrm{C} & 2.61315500 & -0.82303500 & 1.61236600 \\ \mathrm{H} & 2.67505500 & -0.41208700 & 0.60446700 \\ \mathrm{H} & 2.33946200 & -1.87491800 & 1.51189400 \\ \mathrm{C} & 3.95512400 & -0.69284300 & 2.32242900 \\ \mathrm{H} & 3.90826000 & -1.10652300 & 3.33044800 \\ \mathrm{H} & 4.73669700 & -1.22125200 & 1.77861500 \\ \mathrm{H} & 4.25106100 & 0.35344800 & 2.40382500\end{array}$

\section{$\underline{\text { DPTS } \bullet C l ~(T S 7 b) ~}$}

$\begin{array}{lccc}\mathrm{O} & 0.06935700 & 0.26169000 & -0.35402200 \\ \mathrm{~S} & 0.07207900 & 0.10781400 & 1.12733900 \\ \mathrm{C} & 1.81700200 & 0.09415300 & 1.60731200 \\ \mathrm{H} & 1.84603600 & 0.22440600 & 2.68995300 \\ \mathrm{H} & 2.26710200 & 0.96033800 & 1.11962600 \\ \mathrm{C} & 2.47977500 & -1.20310900 & 1.16102500 \\ \mathrm{H} & 2.33538800 & -1.31849900 & 0.08620200 \\ \mathrm{H} & 1.98766700 & -2.04885900 & 1.64486500 \\ \mathrm{C} & 3.96672600 & -1.20198400 & 1.49693700 \\ \mathrm{H} & 4.12492000 & -1.09603400 & 2.57050200\end{array}$




$\begin{array}{lrrr}\mathrm{H} & 4.43863600 & -2.12937600 & 1.17622000 \\ \mathrm{H} & 4.47526700 & -0.37555400 & 0.99998000 \\ \mathrm{C} & -1.78798600 & 2.54729100 & 1.02611800 \\ \mathrm{H} & -1.69934400 & 1.91890500 & 0.13715900 \\ \mathrm{H} & -1.86010800 & 3.58991400 & 0.71175200 \\ \mathrm{C} & -2.99441200 & 2.15450300 & 1.86632500 \\ \mathrm{H} & -2.88143700 & 1.11916100 & 2.18953600 \\ \mathrm{H} & -3.02335500 & 2.76293600 & 2.77034700 \\ \mathrm{C} & -4.27791400 & 2.32228200 & 1.06203500 \\ \mathrm{H} & -5.14439700 & 2.03103200 & 1.65337700 \\ \mathrm{H} & -4.41478500 & 3.35916600 & 0.75318300 \\ \mathrm{H} & -4.25988600 & 1.70451600 & 0.16383800 \\ \mathrm{~S} & -0.19223100 & 2.47299000 & 1.88603600 \\ \mathrm{Cl} & -0.52815400 & 1.46088600 & 3.86454700\end{array}$

\section{$\underline{\text { DPTS } \bullet C I ~(P C 12) ~}$}

$\begin{array}{lccc}\mathrm{O} & 0.17713600 & -1.14238600 & -1.90714300 \\ \mathrm{~S} & 0.21659700 & -1.99249000 & -0.66405900 \\ \mathrm{C} & 1.83346100 & -1.68417600 & 0.08341200 \\ \mathrm{H} & 1.84914500 & -2.27548400 & 1.00133300 \\ \mathrm{H} & 1.85248100 & -0.62604100 & 0.35037200 \\ \mathrm{C} & 2.97756800 & -2.04431800 & -0.85479400 \\ \mathrm{H} & 2.84052100 & -1.50053500 & -1.78980900 \\ \mathrm{H} & 2.92881800 & -3.10830200 & -1.09397100 \\ \mathrm{C} & 4.32886500 & -1.70460800 & -0.23756400 \\ \mathrm{H} & 4.48115500 & -2.24682900 & 0.69633800 \\ \mathrm{H} & 5.14288100 & -1.96334900 & -0.91315600 \\ \mathrm{H} & 4.39790500 & -0.63857100 & -0.01961300 \\ \mathrm{C} & -1.64802700 & 1.21908700 & -0.46332200 \\ \mathrm{H} & -1.75828200 & 0.14966500 & -0.63831700 \\ \mathrm{H} & -1.39369400 & 1.67429200 & -1.42497800 \\ \mathrm{C} & -2.89957600 & 1.83620700 & 0.13530200 \\ \mathrm{H} & -3.05975800 & 1.41850100 & 1.13032800 \\ \mathrm{H} & -2.74522600 & 2.90846300 & 0.26207900 \\ \mathrm{C} & -4.11659200 & 1.57222500 & -0.74419400 \\ \mathrm{H} & -5.01352100 & 2.01497700 & -0.31384700 \\ \mathrm{H} & -3.97814400 & 1.99330700 & -1.74067800 \\ \mathrm{H} & -4.29065500 & 0.50177300 & -0.85690700 \\ \mathrm{~S} & -0.14427600 & 1.48263900 & 0.50249200 \\ \mathrm{Cl} & -0.54146100 & 0.29114900 & 2.11859900\end{array}$

\section{$\underline{\text { DPTS } \bullet C I ~(T S 8) ~}$}




$\begin{array}{lccc}\mathrm{O} & -0.03498300 & 0.91624100 & -1.20113400 \\ \mathrm{~S} & 0.29063300 & 1.07859300 & 0.21905400 \\ \mathrm{C} & 2.09623100 & 0.95022500 & 0.36907400 \\ \mathrm{H} & 2.29950200 & 0.88818200 & 1.43854300 \\ \mathrm{H} & 2.43567900 & 1.91675400 & -0.00616600 \\ \mathrm{C} & 2.68450300 & -0.20121600 & -0.42626600 \\ \mathrm{H} & 2.31768900 & -0.14204800 & -1.45070500 \\ \mathrm{H} & 2.33666000 & -1.14540800 & -0.01038900 \\ \mathrm{C} & 4.20852700 & -0.14128800 & -0.40194200 \\ \mathrm{H} & 4.58861500 & -0.20395600 & 0.61804400 \\ \mathrm{H} & 4.63255000 & -0.96807400 & -0.96918600 \\ \mathrm{H} & 4.57195000 & 0.78857100 & -0.83984600 \\ \mathrm{C} & -1.36234500 & 3.69513300 & -0.39868400 \\ \mathrm{H} & -1.13876600 & 3.17478600 & -1.33019600 \\ \mathrm{H} & -1.49480000 & 4.75440600 & -0.63809800 \\ \mathrm{C} & -2.61954000 & 3.13634400 & 0.25310300 \\ \mathrm{H} & -2.44810300 & 2.09075600 & 0.51931100 \\ \mathrm{H} & -2.80782900 & 3.67005800 & 1.18539600 \\ \mathrm{C} & -3.81881100 & 3.24049400 & -0.68109400 \\ \mathrm{H} & -4.71489700 & 2.83257000 & -0.21586400 \\ \mathrm{H} & -4.01944800 & 4.28010800 & -0.94354500 \\ \mathrm{H} & -3.63883400 & 2.68987500 & -1.60480200 \\ \mathrm{~S} & 0.10320800 & 3.65479200 & 0.65816800 \\ \mathrm{Cl} & -0.15301500 & -0.78589800 & 1.20797200 \\ & & & \end{array}$

\section{$\underline{\text { DPTS••Cl (PC13) }}$}

$\begin{array}{lrrr}\mathrm{O} & -0.61291500 & -0.69815000 & -0.57276100 \\ \mathrm{~S} & -0.02084900 & -0.85701900 & 0.76704400 \\ \mathrm{C} & 1.77627800 & -0.72894500 & 0.53519200 \\ \mathrm{H} & 2.22455400 & -1.07520800 & 1.46645400 \\ \mathrm{H} & 1.92182000 & 0.35030700 & 0.45013200 \\ \mathrm{C} & 2.28797200 & -1.46576600 & -0.68849800 \\ \mathrm{H} & 1.70627600 & -1.15202100 & -1.55469400 \\ \mathrm{H} & 2.12401000 & -2.53520200 & -0.56027500 \\ \mathrm{C} & 3.76988500 & -1.18069000 & -0.90931300 \\ \mathrm{H} & 4.36397700 & -1.49942700 & -0.05236900 \\ \mathrm{H} & 4.13669200 & -1.71038200 & -1.78697400 \\ \mathrm{H} & 3.94586500 & -0.11549100 & -1.06167700 \\ \mathrm{C} & -1.22372400 & 2.36407200 & -0.48529100 \\ \mathrm{H} & -0.81003100 & 1.64777500 & -1.19201000 \\ \mathrm{H} & -1.37553800 & 3.32746900 & -0.97110300 \\ \mathrm{C} & -2.56243100 & 1.83566500 & 0.04446600 \\ \mathrm{H} & -2.38447600 & 0.94709300 & 0.65159700\end{array}$




$\begin{array}{llll}\mathrm{H} & -3.01462900 & 2.58748800 & 0.69244800 \\ \mathrm{C} & -3.49011500 & 1.48252800 & -1.11126700 \\ \mathrm{H} & -4.45483900 & 1.13712800 & -0.74214700 \\ \mathrm{H} & -3.66349900 & 2.34744800 & -1.75266500 \\ \mathrm{H} & -3.05229900 & 0.68795600 & -1.71497500 \\ \mathrm{~S} & -0.08637600 & 2.57773100 & 0.89334900 \\ \mathrm{Cl} & -0.02159300 & -2.92923100 & 1.22967500\end{array}$

\section{$\underline{\mathrm{CH}}_{3} \underline{\mathrm{CH}}_{2} \underline{\mathrm{CH}}_{2} \underline{\mathrm{S}^{\bullet}(\mathbf{P 8 b})}$}

$\begin{array}{llll}\mathrm{C} & -1.21323700 & 2.33210900 & -0.47000800 \\ \mathrm{H} & -0.73275500 & 1.58875700 & -1.11316800 \\ \mathrm{H} & -1.33245200 & 3.22406500 & -1.09233200 \\ \mathrm{C} & -2.55813100 & 1.83262600 & 0.03615700 \\ \mathrm{H} & -2.39624500 & 0.95289500 & 0.66052000 \\ \mathrm{H} & -2.99814500 & 2.59453300 & 0.68106400 \\ \mathrm{C} & -3.50150600 & 1.50114200 & -1.11391700 \\ \mathrm{H} & -4.46275600 & 1.14489100 & -0.74617100 \\ \mathrm{H} & -3.68464800 & 2.37973200 & -1.73415500 \\ \mathrm{H} & -3.07913000 & 0.72522600 & -1.75400600 \\ \mathrm{~S} & -0.01875300 & 2.75381300 & 0.80466300\end{array}$

\section{$\mathrm{CH}_{3} \underline{\mathrm{CH}}_{2} \underline{\mathrm{CH}}_{2} \underline{\mathrm{S}(=\mathrm{O}) \mathrm{Cl} \text { (P8a) }}$}

$\begin{array}{lrrr}\mathrm{O} & -0.83770000 & 1.54355400 & -0.80946200 \\ \mathrm{~S} & -0.90826800 & 0.72337500 & 0.40699200 \\ \mathrm{C} & 0.80491600 & 0.24154400 & 0.77643600 \\ \mathrm{H} & 0.74760900 & -0.53234600 & 1.54178900 \\ \mathrm{H} & 1.21492500 & 1.15099300 & 1.22463800 \\ \mathrm{C} & 1.59900100 & -0.17761900 & -0.44669000 \\ \mathrm{H} & 1.49722600 & 0.59355000 & -1.20960600 \\ \mathrm{H} & 1.17037500 & -1.09292800 & -0.85344300 \\ \mathrm{C} & 3.06720700 & -0.39159500 & -0.09438200 \\ \mathrm{H} & 3.18196600 & -1.16731500 & 0.66325400 \\ \mathrm{H} & 3.63250500 & -0.69578300 & -0.97364300 \\ \mathrm{H} & 3.51553800 & 0.52422700 & 0.29190700 \\ \mathrm{Cl} & -1.56194100 & -1.21981300 & -0.12548600\end{array}$

\section{$\underline{\text { DPTS } \bullet C l \text { (TS9) }}$}

$\begin{array}{llll}\mathrm{O} & 1.08361800 & -0.29648400 & -1.31788100 \\ \mathrm{~S} & 0.75445100 & -0.42212700 & 0.10199100 \\ \mathrm{C} & 3.01357700 & -0.66324400 & 0.98380200 \\ \mathrm{H} & 3.02260800 & -1.74595500 & 1.01632500 \\ \mathrm{H} & 2.84624100 & -0.17605900 & 1.93766700 \\ \mathrm{C} & 3.85519700 & 0.02050600 & -0.03463100\end{array}$




$\begin{array}{lccc}\mathrm{H} & 3.56113200 & 1.06775700 & -0.11315800 \\ \mathrm{H} & 3.69205900 & -0.43364400 & -1.01184900 \\ \mathrm{C} & 5.34235600 & -0.07382700 & 0.34019900 \\ \mathrm{H} & 5.66275600 & -1.11332000 & 0.40407400 \\ \mathrm{H} & 5.95198400 & 0.42485200 & -0.41244100 \\ \mathrm{H} & 5.53365500 & 0.39906300 & 1.30294400 \\ \mathrm{C} & -0.36446700 & 2.34873300 & -0.31587900 \\ \mathrm{H} & -0.43178300 & 1.67285100 & -1.17048700 \\ \mathrm{H} & 0.20097400 & 3.22313400 & -0.63591100 \\ \mathrm{C} & -1.73666500 & 2.73620500 & 0.21285900 \\ \mathrm{H} & -2.24166400 & 1.84119500 & 0.57302700 \\ \mathrm{H} & -1.62028000 & 3.40676900 & 1.06606500 \\ \mathrm{C} & -2.56075600 & 3.40936900 & -0.87838500 \\ \mathrm{H} & -3.53916500 & 3.70405800 & -0.50231000 \\ \mathrm{H} & -2.06181100 & 4.30372600 & -1.25421700 \\ \mathrm{H} & -2.71296300 & 2.73147900 & -1.71877900 \\ \mathrm{~S} & 0.66640000 & 1.51492300 & 0.93712300 \\ \mathrm{Cl} & -1.43729800 & -0.82778300 & 0.03820800\end{array}$

\section{DPTS • Cl (PC14)}

$\begin{array}{lrrr}\mathrm{O} & -0.32309200 & -1.12991400 & -0.54693300 \\ \mathrm{~S} & -0.54471900 & -1.27699300 & 0.89285200 \\ \mathrm{C} & 2.59675100 & -1.26562700 & 0.95526200 \\ \mathrm{H} & 2.53504200 & -2.26788000 & 0.55496500 \\ \mathrm{H} & 2.77091000 & -1.16364400 & 2.01672500 \\ \mathrm{C} & 2.81158600 & -0.11437600 & 0.03970000 \\ \mathrm{H} & 2.52520100 & 0.81323900 & 0.54012000 \\ \mathrm{H} & 2.16809500 & -0.22100600 & -0.83649300 \\ \mathrm{C} & 4.27309300 & -0.00727300 & -0.42247900 \\ \mathrm{H} & 4.57619500 & -0.91249000 & -0.94804700 \\ \mathrm{H} & 4.40653000 & 0.83944900 & -1.09591000 \\ \mathrm{H} & 4.93911600 & 0.12434000 & 0.42997600 \\ \mathrm{C} & -0.55773100 & 1.64105300 & 0.23920000 \\ \mathrm{H} & 0.09024200 & 1.19848100 & -0.51719900 \\ \mathrm{H} & -0.13562900 & 2.60709000 & 0.52256600 \\ \mathrm{C} & -1.98524400 & 1.77655300 & -0.26315500 \\ \mathrm{H} & -2.36573200 & 0.78814400 & -0.51974800 \\ \mathrm{H} & -2.61630900 & 2.16465600 & 0.53740800 \\ \mathrm{C} & -2.04039100 & 2.69024700 & -1.48193800 \\ \mathrm{H} & -3.06069400 & 2.78827200 & -1.84893600 \\ \mathrm{H} & -1.67187400 & 3.68860600 & -1.24195300 \\ \mathrm{H} & -1.42922000 & 2.29199200 & -2.29233400 \\ \mathrm{~S} & -0.38066600 & 0.64690500 & 1.74989600\end{array}$


Cl $\quad-2.64100400 \quad-1.60377100 \quad 1.11097400$

\section{$\underline{\mathrm{CH}}_{3} \underline{\mathrm{CH}}_{2} \underline{\mathrm{CH}} \underline{2} \underline{\mathrm{SS}(\mathrm{O})-\mathrm{Cl} \text { (P9a) }}$}

$\begin{array}{lccc}\mathrm{O} & -0.28001600 & -1.15669400 & -0.52118500 \\ \mathrm{~S} & -0.55638700 & -1.27750200 & 0.91076300 \\ \mathrm{C} & -0.55480900 & 1.63504300 & 0.22742800 \\ \mathrm{H} & 0.08656200 & 1.18906100 & -0.53171600 \\ \mathrm{H} & -0.13019800 & 2.60097400 & 0.50631700 \\ \mathrm{C} & -1.98559300 & 1.77154800 & -0.26501600 \\ \mathrm{H} & -2.36466000 & 0.78566000 & -0.53285400 \\ \mathrm{H} & -2.61437400 & 2.14801100 & 0.54285000 \\ \mathrm{C} & -2.04895900 & 2.70005900 & -1.47232100 \\ \mathrm{H} & -3.07084100 & 2.79639100 & -1.83522500 \\ \mathrm{H} & -1.68581500 & 3.69736700 & -1.22048900 \\ \mathrm{H} & -1.43757900 & 2.31579700 & -2.28921500 \\ \mathrm{~S} & -0.36712000 & 0.64661900 & 1.74197000 \\ \mathrm{Cl} & -2.65227500 & -1.58101300 & 1.07939400\end{array}$

\section{Propyl radical $\left(\mathrm{CH}_{3} \mathrm{CH}_{2} \mathrm{C}^{*} \mathrm{H}_{2},(\mathrm{P} 9 \mathrm{~b})\right)$}

$\begin{array}{llrc}\mathrm{C} & 2.61952200 & -1.29154900 & 0.93938100 \\ \mathrm{H} & 2.61793023 & -1.15006603 & 2.00931297 \\ \mathrm{H} & 2.31021597 & -2.25331723 & 0.55593057 \\ \mathrm{C} & 2.82390400 & -0.14815900 & 0.01362500 \\ \mathrm{H} & 2.45169500 & 0.77109000 & 0.47354100 \\ \mathrm{H} & 2.21399600 & -0.28373000 & -0.89035700 \\ \mathrm{C} & 4.28331700 & 0.02591200 & -0.39570200 \\ \mathrm{H} & 4.65477300 & -0.87705200 & -0.88158200 \\ \mathrm{H} & 4.40614600 & 0.85662400 & -1.08929400 \\ \mathrm{H} & 4.90757700 & 0.21480100 & 0.47756500\end{array}$


Table S7: The calculated bimolecular rate coefficients (in $\mathrm{cm}^{3}$ molecule-1 $\mathrm{s}^{-1}$ ) for all the possible $\mathrm{H}$-abstraction and substitution pathways involving Eckart tunneling contributions associated with the DPTS $+{ }^{\circ} \mathrm{Cl}$ reaction in the temperatures between 200 and $320 \mathrm{~K}$ and at 1 atm pressure.

\begin{tabular}{|c|c|c|c|c|c|c|c|c|}
\hline $\mathrm{T}(\mathrm{K})$ & $\mathrm{k}_{\mathrm{TS} 1 \mathrm{a}}$ & $\mathrm{k}_{\mathrm{TS} 1 \mathrm{~b}}$ & $\mathrm{k}_{\mathrm{TS} 2 \mathrm{a}}$ & $\mathrm{k}_{\mathrm{TS} 2 \mathrm{~b}}$ & $\mathrm{k}_{\mathrm{TS} 3 \mathrm{a}}$ & $\mathrm{k}_{\mathrm{TS} 3 \mathrm{~b}}$ & $\mathrm{k}_{\mathrm{TS3c}}$ & $\mathrm{k}_{\mathrm{TS} 4 \mathrm{a}}$ \\
\hline 200 & $9.39 \times 10^{-12}$ & $2.82 \times 10^{-12}$ & $8.10 \times 10^{-12}$ & $4.67 \times 10^{-11}$ & $4.94 \times 10^{-12}$ & $1.34 \times 10^{-12}$ & $2.25 \times 10^{-12}$ & $8.16 \times 10^{-12}$ \\
\hline 220 & $9.48 \times 10^{-12}$ & $2.85 \times 10^{-12}$ & $7.55 \times 10^{-12}$ & $4.71 \times 10^{-11}$ & $4.95 \times 10^{-12}$ & $1.46 \times 10^{-12}$ & $2.38 \times 10^{-12}$ & $7.66 \times 10^{-12}$ \\
\hline 240 & $9.55 \times 10^{-12}$ & $2.88 \times 10^{-12}$ & $7.01 \times 10^{-12}$ & $4.74 \times 10^{-11}$ & $4.99 \times 10^{-12}$ & $1.61 \times 10^{-12}$ & $2.54 \times 10^{-12}$ & $7.19 \times 10^{-12}$ \\
\hline 250 & $9.58 \times 10^{-12}$ & $2.89 \times 10^{-12}$ & $6.78 \times 10^{-12}$ & $4.74 \times 10^{-11}$ & $5.02 \times 10^{-12}$ & $1.69 \times 10^{-12}$ & $2.62 \times 10^{-12}$ & $6.98 \times 10^{-12}$ \\
\hline 260 & $9.59 \times 10^{-12}$ & $2.90 \times 10^{-12}$ & $6.58 \times 10^{-12}$ & $4.74 \times 10^{-11}$ & $5.05 \times 10^{-12}$ & $1.78 \times 10^{-12}$ & $2.71 \times 10^{-12}$ & $6.81 \times 10^{-12}$ \\
\hline 280 & $9.61 \times 10^{-12}$ & $2.91 \times 10^{-12}$ & $6.29 \times 10^{-12}$ & $4.72 \times 10^{-11}$ & $5.13 \times 10^{-12}$ & $1.96 \times 10^{-12}$ & $2.90 \times 10^{-12}$ & $6.54 \times 10^{-12}$ \\
\hline 298 & $9.57 \times 10^{-12}$ & $2.92 \times 10^{-12}$ & $6.12 \times 10^{-12}$ & $4.69 \times 10^{-11}$ & $5.22 \times 10^{-12}$ & $2.14 \times 10^{-12}$ & $3.08 \times 10^{-12}$ & $6.38 \times 10^{-12}$ \\
\hline 300 & $9.57 \times 10^{-12}$ & $2.92 \times 10^{-12}$ & $6.11 \times 10^{-12}$ & $4.68 \times 10^{-11}$ & $5.23 \times 10^{-12}$ & $2.16 \times 10^{-12}$ & $3.10 \times 10^{-12}$ & $6.37 \times 10^{-12}$ \\
\hline
\end{tabular}

\begin{tabular}{|c|c|c|c|c|c|c|c|}
\hline $\mathrm{k}_{\mathrm{TS} 4 \mathrm{~b}}$ & $\mathrm{k}_{\mathrm{TS} 5 \mathrm{a}}$ & $\mathrm{k}_{\mathrm{TS} 5 \mathrm{~b}}$ & $\mathrm{k}_{\mathrm{TS} 6 \mathrm{a}}$ & $\mathrm{k}_{\mathrm{TS} 6 \mathrm{~b}}$ & $\mathrm{k}_{\mathrm{TS} 6 \mathrm{c}}$ & $\mathrm{k}_{\text {TS7a }}$ & $\mathrm{k}_{\mathrm{TS} 7 \mathrm{~b}}$ \\
\hline $5.45 \times 10^{-11}$ & $9.39 \times 10^{-12}$ & $4.72 \times 10^{-11}$ & $4.73 \times 10^{-12}$ & $5.86 \times 10^{-12}$ & $7.67 \times 10^{-12}$ & $4.71 \times 10^{-13}$ & $9.38 \times 10^{-12}$ \\
\hline $5.45 \times 10^{-11}$ & $9.48 \times 10^{-12}$ & $4.76 \times 10^{-11}$ & $4.77 \times 10^{-12}$ & $5.48 \times 10^{-12}$ & $6.61 \times 10^{-12}$ & $4.76 \times 10^{-13}$ & $9.47 \times 10^{-12}$ \\
\hline $5.44 \times 10^{-11}$ & $9.55 \times 10^{-12}$ & $4.78 \times 10^{-11}$ & $4.81 \times 10^{-12}$ & $5.07 \times 10^{-12}$ & $5.45 \times 10^{-12}$ & $4.81 \times 10^{-13}$ & $9.54 \times 10^{-12}$ \\
\hline $5.42 \times 10^{-11}$ & $9.58 \times 10^{-12}$ & $4.79 \times 10^{-11}$ & $4.83 \times 10^{-12}$ & $4.89 \times 10^{-12}$ & $4.89 \times 10^{-12}$ & $4.83 \times 10^{-13}$ & $9.57 \times 10^{-12}$ \\
\hline $5.41 \times 10^{-11}$ & $9.60 \times 10^{-12}$ & $4.78 \times 10^{-11}$ & $4.84 \times 10^{-12}$ & $4.72 \times 10^{-12}$ & $4.39 \times 10^{-12}$ & $4.85 \times 10^{-13}$ & $9.59 \times 10^{-12}$ \\
\hline $5.37 \times 10^{-11}$ & $9.61 \times 10^{-12}$ & $4.75 \times 10^{-11}$ & $4.86 \times 10^{-12}$ & $4.45 \times 10^{-12}$ & $3.54 \times 10^{-12}$ & $4.88 \times 10^{-13}$ & $9.61 \times 10^{-12}$ \\
\hline $5.33 \times 10^{-11}$ & $9.58 \times 10^{-12}$ & $4.70 \times 10^{-11}$ & $4.86 \times 10^{-12}$ & $4.27 \times 10^{-12}$ & $2.98 \times 10^{-12}$ & $4.90 \times 10^{-13}$ & $9.58 \times 10^{-12}$ \\
\hline $5.33 \times 10^{-11}$ & $9.58 \times 10^{-12}$ & $4.69 \times 10^{-11}$ & $4.86 \times 10^{-12}$ & $4.26 \times 10^{-12}$ & $2.93 \times 10^{-12}$ & $4.91 \times 10^{-13}$ & $9.58 \times 10^{-12}$ \\
\hline
\end{tabular}

\begin{tabular}{|c|c|c|}
\hline $\mathrm{k}_{\mathrm{TS} 8}$ & $\mathrm{k}_{\mathrm{TS} 9}$ & $k_{\text {total }}^{\mathrm{a}}$ \\
\hline $5.81 \times 10^{-10}$ & $9.43 \times 10^{-13}$ & $8.04 \times 10^{-10}$ \\
\hline $5.86 \times 10^{-10}$ & $9.54 \times 10^{-13}$ & $8.09 \times 10^{-10}$ \\
\hline $5.91 \times 10^{-10}$ & $9.63 \times 10^{-13}$ & $8.12 \times 10^{-10}$ \\
\hline $5.93 \times 10^{-10}$ & $9.67 \times 10^{-13}$ & $8.13 \times 10^{-10}$ \\
\hline
\end{tabular}




\begin{tabular}{|l|l|l|}
\hline $5.95 \times 10^{-10}$ & $9.72 \times 10^{-13}$ & $8.14 \times 10^{-10}$ \\
\hline $5.98 \times 10^{-10}$ & $9.79 \times 10^{-13}$ & $8.15 \times 10^{-10}$ \\
\hline $5.99 \times 10^{-10}$ & $9.84 \times 10^{-13}$ & $8.14 \times 10^{-10}$ \\
\hline $5.99 \times 10^{-10}$ & $9.85 \times 10^{-13}$ & $8.14 \times 10^{-10}$ \\
\hline
\end{tabular}

a The overall rate coefficient $\left(k_{\text {total }}\right)$ for the DPTS $+{ }^{\circ} \mathrm{Cl}$ reaction was calculated by summing the values for all the individual reaction pathways at the indicated temperature.

Table S8: The calculated bimolecular rate coefficients (in $\mathrm{cm}^{3}$ molecule ${ }^{-1} \mathrm{~s}^{-1}$ ) for all the possible abstraction and substitution pathways without tunneling for the DPTS $+{ }^{\circ} \mathrm{Cl}$ reaction in the temperatures between 200 and $320 \mathrm{~K}$ and at 1 atm pressure.

\begin{tabular}{|c|c|c|c|c|c|c|c|}
\hline $\mathrm{T}(\mathrm{K})$ & $\mathrm{k}_{\text {TS1a }}$ & $\mathrm{k}_{\mathrm{TS} 1 \mathrm{~b}}$ & $\mathrm{k}_{\mathrm{TS} 2 \mathrm{a}}$ & $\mathrm{k}_{\mathrm{TS} 2 \mathrm{~b}}$ & $\mathrm{k}_{\mathrm{TS} 3 \mathrm{a}}$ & $\mathrm{k}_{\mathrm{TS} 3 \mathrm{~b}}$ & $\mathrm{k}_{\text {TS3c }}$ \\
\hline 200 & $9.39 \times 10^{-12}$ & $9.42 \times 10^{-13}$ & $7.75 \times 10^{-12}$ & $4.67 \times 10^{-11}$ & $4.52 \times 10^{-12}$ & $4.06 \times 10^{-13}$ & $1.18 \times 10^{-12}$ \\
\hline 220 & $9.48 \times 10^{-12}$ & $9.52 \times 10^{-13}$ & $6.87 \times 10^{-12}$ & $4.71 \times 10^{-11}$ & $4.59 \times 10^{-12}$ & $4.38 \times 10^{-13}$ & $1.36 \times 10^{-12}$ \\
\hline 240 & $9.55 \times 10^{-12}$ & $9.60 \times 10^{-13}$ & $5.98 \times 10^{-12}$ & $4.74 \times 10^{-11}$ & $4.69 \times 10^{-12}$ & $4.74 \times 10^{-13}$ & $1.57 \times 10^{-12}$ \\
\hline 250 & $9.58 \times 10^{-12}$ & $9.64 \times 10^{-13}$ & $5.58 \times 10^{-12}$ & $4.74 \times 10^{-11}$ & $4.74 \times 10^{-12}$ & $4.94 \times 10^{-13}$ & $1.67 \times 10^{-12}$ \\
\hline 260 & $9.59 \times 10^{-12}$ & $9.67 \times 10^{-13}$ & $5.24 \times 10^{-12}$ & $4.74 \times 10^{-11}$ & $4.80 \times 10^{-12}$ & $5.14 \times 10^{-13}$ & $1.79 \times 10^{-12}$ \\
\hline 280 & $9.61 \times 10^{-12}$ & $9.72 \times 10^{-13}$ & $4.73 \times 10^{-12}$ & $4.72 \times 10^{-11}$ & $4.92 \times 10^{-12}$ & $5.55 \times 10^{-13}$ & $2.02 \times 10^{-12}$ \\
\hline 298 & $9.57 \times 10^{-12}$ & $9.75 \times 10^{-13}$ & $4.46 \times 10^{-12}$ & $4.69 \times 10^{-11}$ & $5.03 \times 10^{-12}$ & $5.91 \times 10^{-13}$ & $2.24 \times 10^{-12}$ \\
\hline 300 & $9.57 \times 10^{-12}$ & $9.76 \times 10^{-13}$ & $4.44 \times 10^{-12}$ & $4.68 \times 10^{-11}$ & $5.04 \times 10^{-12}$ & $5.95 \times 10^{-13}$ & $2.26 \times 10^{-12}$ \\
\hline
\end{tabular}




\begin{tabular}{|c|c|c|c|c|c|c|c|}
\hline $\mathrm{k}_{\text {TS4a }}$ & $\mathrm{k}_{\text {TS4b }}$ & $\mathrm{k}_{\text {TS5a }}$ & $\mathrm{k}_{\text {TS5b }}$ & $\mathrm{k}_{\text {TS6a }}$ & $\mathrm{k}_{\text {TS6b }}$ & $\mathrm{k}_{\text {TS6c }}$ & $\mathrm{k}_{\text {TS7a }}$ \\
\hline $8.15 \times 10^{-12}$ & $5.43 \times 10^{-11}$ & $9.39 \times 10^{-12}$ & $4.72 \times 10^{-11}$ & $7.57 \times 10^{-13}$ & $3.48 \times 10^{-12}$ & $4.17 \times 10^{-12}$ & $3.77 \times 10^{-14}$ \\
\hline $7.63 \times 10^{-12}$ & $5.43 \times 10^{-11}$ & $9.48 \times 10^{-12}$ & $4.76 \times 10^{-11}$ & $7.64 \times 10^{-13}$ & $3.31 \times 10^{-12}$ & $3.77 \times 10^{-12}$ & $3.81 \times 10^{-14}$ \\
\hline $7.15 \times 10^{-12}$ & $5.41 \times 10^{-11}$ & $9.55 \times 10^{-12}$ & $4.78 \times 10^{-11}$ & $7.70 \times 10^{-13}$ & $3.11 \times 10^{-12}$ & $3.27 \times 10^{-12}$ & $3.84 \times 10^{-14}$ \\
\hline $6.94 \times 10^{-12}$ & $5.39 \times 10^{-11}$ & $9.58 \times 10^{-12}$ & $4.78 \times 10^{-11}$ & $7.73 \times 10^{-13}$ & $3.02 \times 10^{-12}$ & $3.01 \times 10^{-12}$ & $3.86 \times 10^{-14}$ \\
\hline $6.76 \times 10^{-12}$ & $5.38 \times 10^{-11}$ & $9.59 \times 10^{-12}$ & $4.78 \times 10^{-11}$ & $7.75 \times 10^{-13}$ & $2.93 \times 10^{-12}$ & $2.75 \times 10^{-12}$ & $3.88 \times 10^{-14}$ \\
\hline $6.48 \times 10^{-12}$ & $5.34 \times 10^{-11}$ & $9.61 \times 10^{-12}$ & $4.75 \times 10^{-11}$ & $7.79 \times 10^{-13}$ & $2.79 \times 10^{-12}$ & $2.30 \times 10^{-12}$ & $3.91 \times 10^{-14}$ \\
\hline $6.32 \times 10^{-12}$ & $5.30 \times 10^{-11}$ & $9.58 \times 10^{-12}$ & $4.68 \times 10^{-11}$ & $7.81 \times 10^{-13}$ & $2.70 \times 10^{-12}$ & $1.98 \times 10^{-12}$ & $3.93 \times 10^{-14}$ \\
\hline $6.31 \times 10^{-12}$ & $5.29 \times 10^{-11}$ & $9.57 \times 10^{-12}$ & $4.67 \times 10^{-11}$ & $7.81 \times 10^{-13}$ & $2.69 \times 10^{-12}$ & $1.95 \times 10^{-12}$ & $3.93 \times 10^{-14}$ \\
\hline
\end{tabular}

\begin{tabular}{|c|c|c|}
\hline $\mathrm{k}_{\mathrm{TS} 7 \mathrm{~b}}$ & $\mathrm{k}_{\mathrm{TS} 8}$ & $\mathrm{k}_{\mathrm{TS} 9}$ \\
\hline $9.38 \times 10^{-12}$ & $5.81 \times 10^{-10}$ & $9.43 \times 10^{-16}$ \\
\hline $9.47 \times 10^{-12}$ & $5.86 \times 10^{-10}$ & $9.54 \times 10^{-16}$ \\
\hline $9.54 \times 10^{-12}$ & $5.91 \times 10^{-10}$ & $9.63 \times 10^{-16}$ \\
\hline $9.57 \times 10^{-12}$ & $5.93 \times 10^{-10}$ & $9.68 \times 10^{-16}$ \\
\hline $9.59 \times 10^{-12}$ & $5.95 \times 10^{-10}$ & $9.72 \times 10^{-16}$ \\
\hline $9.60 \times 10^{-12}$ & $5.98 \times 10^{-10}$ & $9.80 \times 10^{-16}$ \\
\hline $9.58 \times 10^{-12}$ & $5.99 \times 10^{-10}$ & $9.86 \times 10^{-16}$ \\
\hline $9.58 \times 10^{-12}$ & $5.99 \times 10^{-10}$ & $9.86 \times 10^{-16}$ \\
\hline
\end{tabular}


Table S9: The calculated branching ratios for all the abstraction and addition paths involved in the PSIA $+\bullet$ OH reaction in the temperatures between 200 and $320 \mathrm{~K}$ and at 760 Torr pressure.

\begin{tabular}{|c|c|c|c|c|c|c|c|c|c|c|c|c|c|c|c|}
\hline $\mathrm{T}(\mathrm{K})$ & TS1a & TS1b & TS2a & TS2b & TS3a & TS3b & TS3c & TS4a & $\mathrm{TS} 4 \mathrm{~b}$ & TS5a & TS5b & TS6a & TS6b & TS6c & $\mathrm{TS} 7 \mathrm{a}$ \\
\hline 200 & 1.2 & 0.4 & 1.0 & 5.8 & 0.6 & 0.2 & 0.3 & 1.0 & 6.8 & 1.2 & 5.9 & 0.6 & 0.7 & 1.0 & 0.1 \\
\hline 220 & 1.2 & 0.4 & 0.9 & 5.8 & 0.6 & 0.2 & 0.3 & 0.9 & 6.7 & 1.2 & 5.9 & 0.6 & 0.7 & 0.8 & 0.1 \\
\hline 240 & 1.2 & 0.4 & 0.9 & 5.8 & 0.6 & 0.2 & 0.3 & 0.9 & 6.7 & 1.2 & 5.9 & 0.6 & 0.6 & 0.7 & 0.1 \\
\hline 250 & 1.2 & 0.4 & 0.8 & 5.8 & 0.6 & 0.2 & 0.3 & 0.9 & 6.7 & 1.2 & 5.9 & 0.6 & 0.6 & 0.6 & 0.1 \\
\hline 260 & 1.2 & 0.4 & 0.8 & 5.8 & 0.6 & 0.2 & 0.3 & 0.8 & 6.6 & 1.2 & 5.9 & 0.6 & 0.6 & 0.5 & 0.1 \\
\hline 280 & 1.2 & 0.4 & 0.8 & 5.8 & 0.6 & 0.2 & 0.4 & 0.8 & 6.6 & 1.2 & 5.8 & 0.6 & 0.5 & 0.4 & 0.1 \\
\hline 298 & 1.2 & 0.4 & 0.8 & 5.8 & 0.6 & 0.3 & 0.4 & 0.8 & 6.5 & 1.2 & 5.8 & 0.6 & 0.5 & 0.4 & 0.1 \\
\hline 300 & 1.2 & 0.4 & 0.8 & 5.8 & 0.6 & 0.3 & 0.4 & 0.8 & 6.5 & 1.2 & 5.8 & 0.6 & 0.5 & 0.4 & 0.1 \\
\hline
\end{tabular}

\begin{tabular}{|c|c|c|}
\hline TS7b & TS8 & TS9 \\
\hline 1.2 & 72.2 & 0.1 \\
\hline 1.2 & 72.5 & 0.1 \\
\hline 1.2 & 72.8 & 0.1 \\
\hline 1.2 & 72.9 & 0.1 \\
\hline 1.2 & 73.1 & 0.1 \\
\hline 1.2 & 73.3 & 0.1 \\
\hline 1.2 & 73.6 & 0.1 \\
\hline 1.2 & 73.6 & 0.1 \\
\hline
\end{tabular}



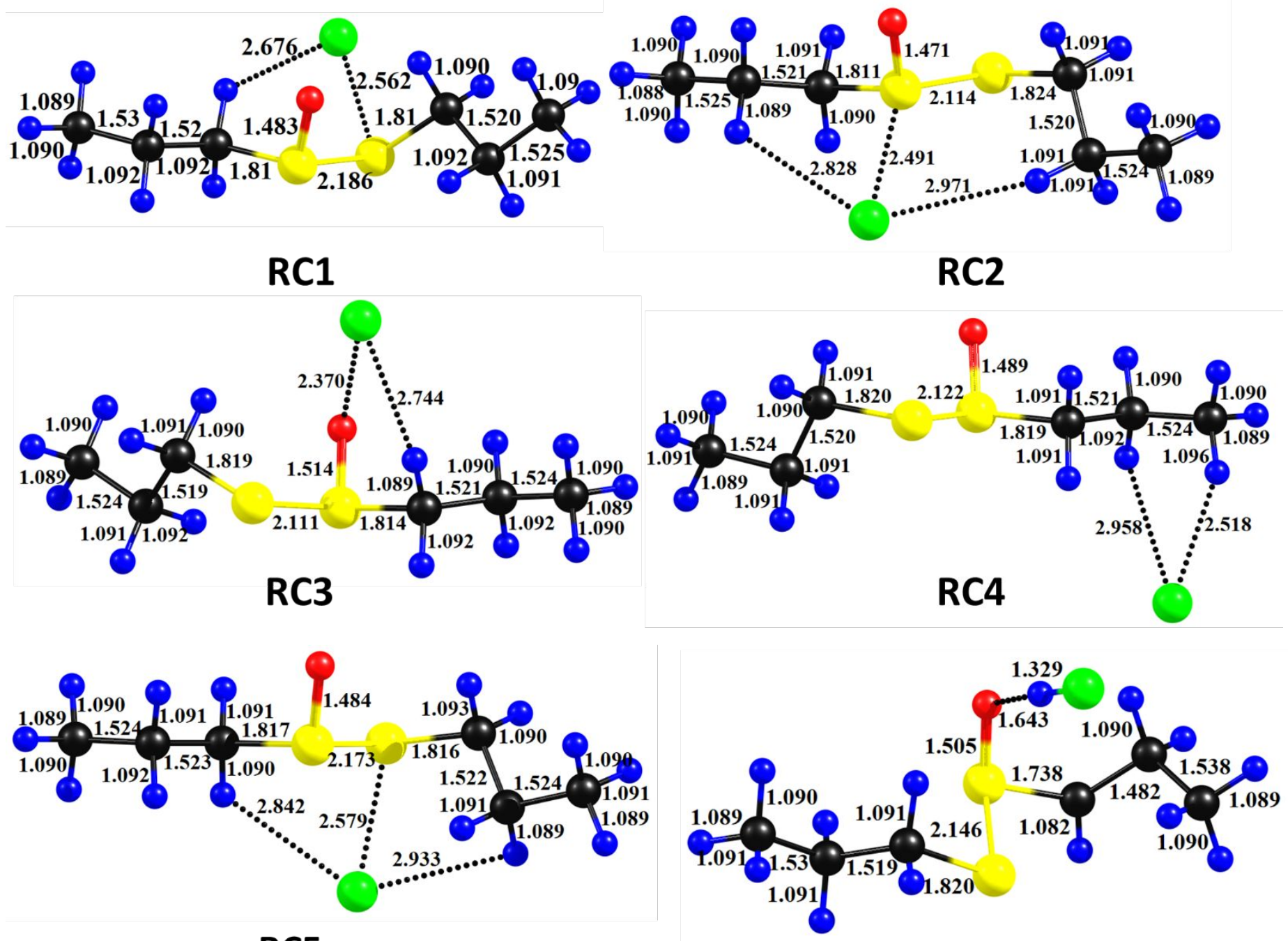

\section{RC5}
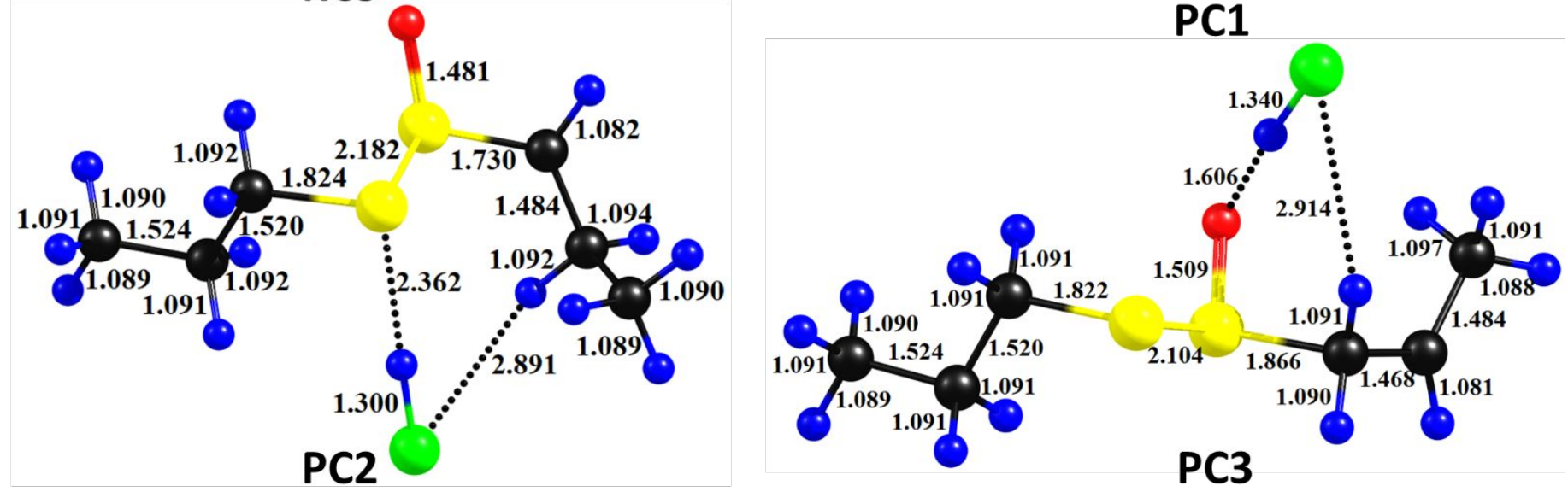

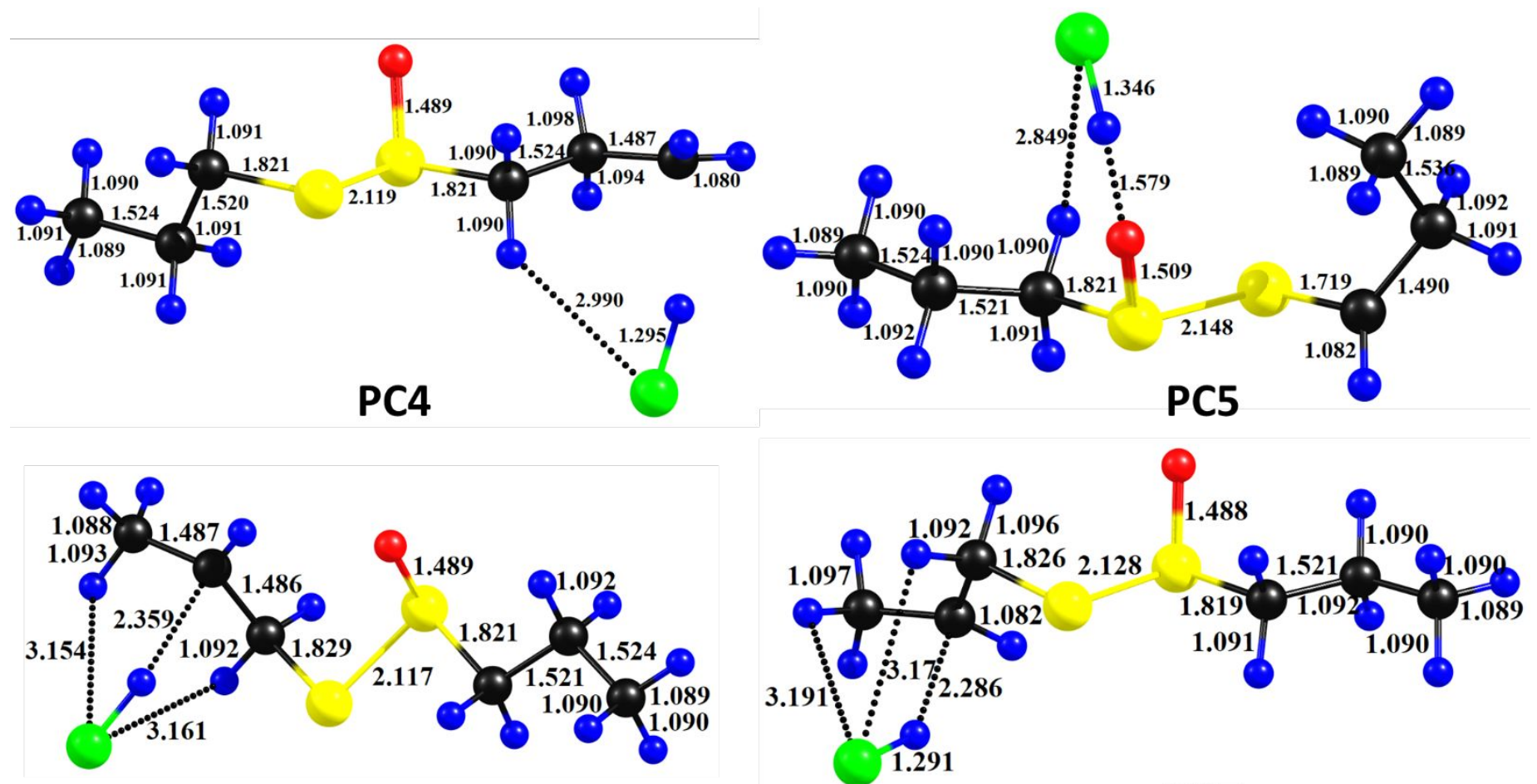

PC6

PC7
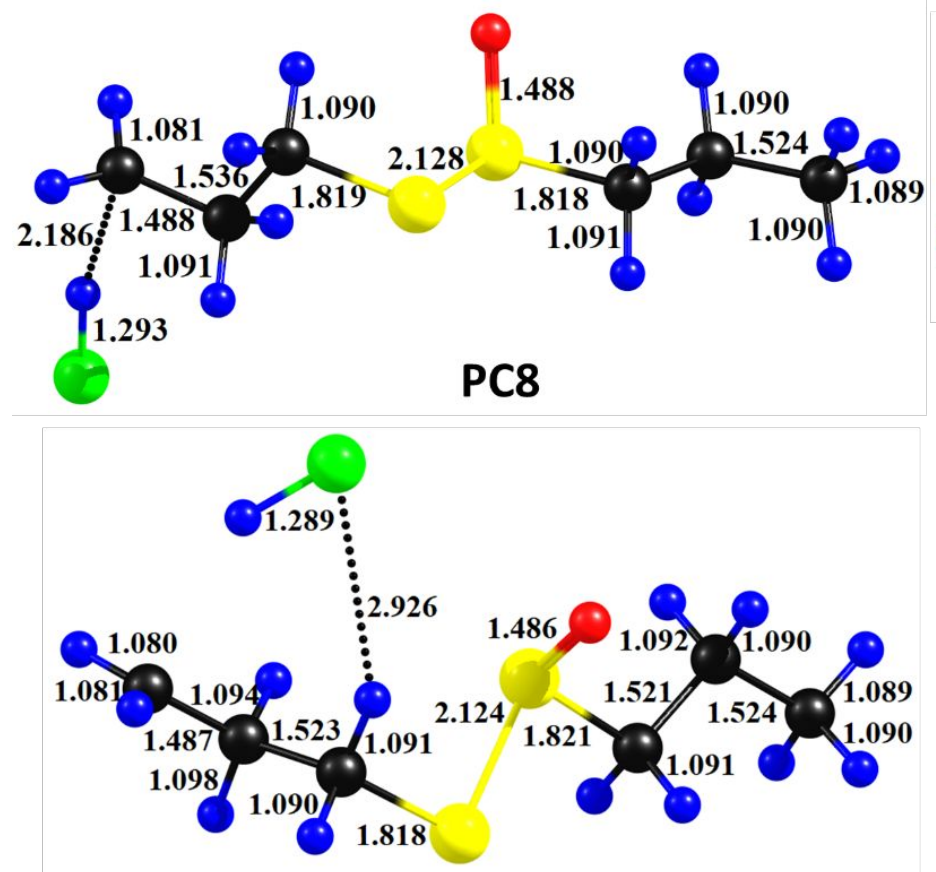

PC10

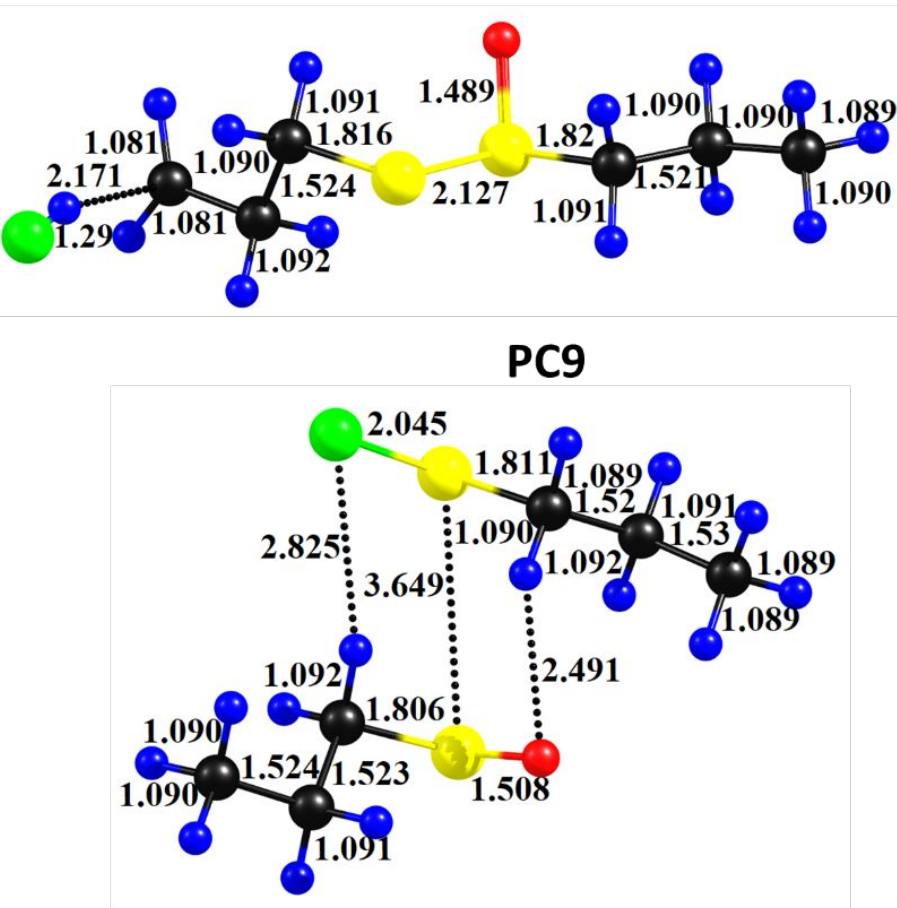

PC11 


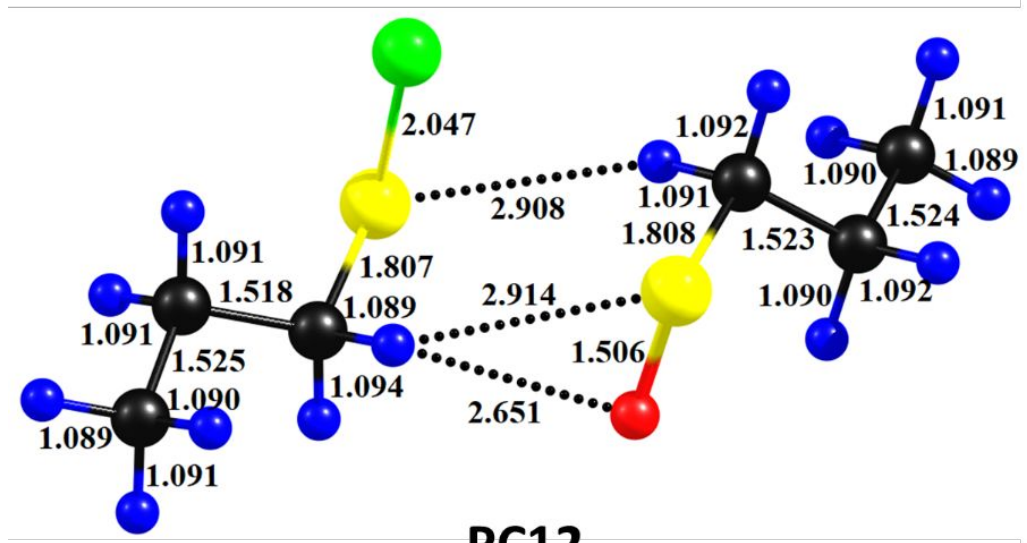

PC12

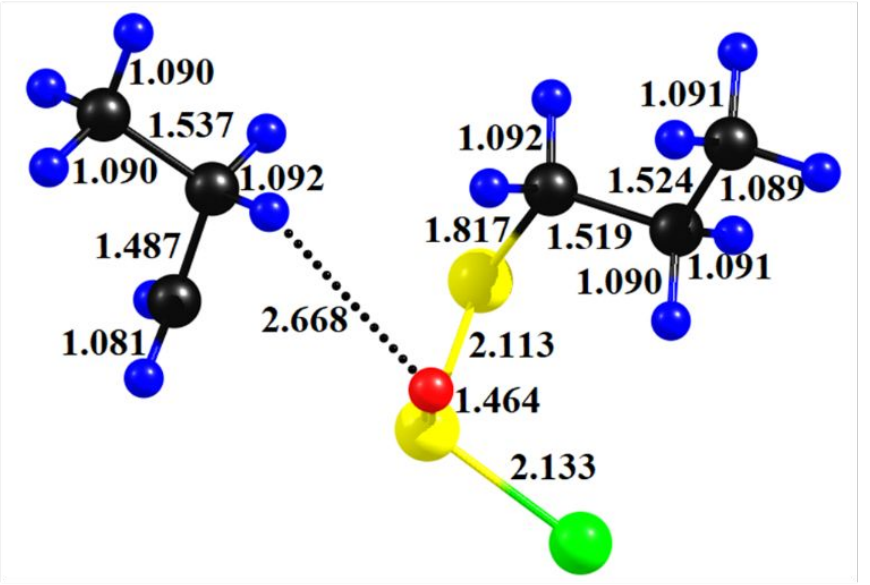

\section{PC14}

Figure S1. The M06-2X/aug-cc-pVTZ level optimized molecular geometries of the pre-reactive and post-reactive complexes involved in the DPTS $+{ }^{\circ} \mathrm{Cl}$ reaction. The black, yellow, red, green, and blue colors represent carbon, sulfur, oxygen, chlorine, and hydrogen atoms, respectively. The bond lengths $(\AA)$ provided on the structures were obtained at the M06-2X/aug-cc-pVTZ level. 